\title{
The Chern-Simons Fermi Liquid Description of Fractional Quantum Hall States
}

\author{
Steven H. Simon \\ Bell Laboratories, Lucent Technologies \\ Murray Hill, NJ 07974
}

\begin{abstract}
The composite fermion picture has had a remarkable number of recent successes both in the description of the fractional quantized Hall states and in the description of the even denominator Fermi-liquid like states. In this chapter, we give an introductory account of the Chern-Simons fermion theory, focusing on the description of the even denominator states as unusual Fermi liquids.
\end{abstract}

\section{Contents}

1 Introduction

2 Introduction to Chern-Simons Fermions 6

2.1 Quantum Hall Effect Basics . . . . . . . . . . . . . . . 6 2.1.1 Integer Quantized Hall Effect : Single Electron Physics 6

2.1.2 Fractional Quantized Hall Effect : Interactions . . . . . 9

2.2 Chern-Simons Transformation . . . . . . . . . . . . . . . . 10

2.3 Mean Field Theory . . . . . . . . . . . . . . . . . . . . . . 12

2.3.1 Even Denominator Fractions . . . . . . . . . . . . 13

2.3.2 Jain Series of Fractional Hall States . . . . . . . . . . . 14

3 RPA 16

3.1 Chern-Simons RPA Basics . . . . . . . . . . . . . . . . 17

3.2 Electromagnetic Response $K_{\mu \nu} \ldots \ldots \ldots \ldots \ldots \ldots$

3.3 Hartree Part of the Coulomb Interaction : Separating $\Pi^{v}$. . . 21

3.4 Relation of Response to Resistivity and Conductivity . . . . . . 23

3.5 General Theme and Simple Example of RPA . . . . . . . . . . 24

3.6 Chern-Simon RPA (Again) _ . . . . . . . . . . . 25

3.7 Results of Chern-Simons RPA at $\nu=\frac{1}{2 m} \quad \ldots \ldots \ldots \ldots 27$

3.8 Sum Rules . . . . . . . . . . . . . . . . . . . . . . . . . 28

3.9 Energy Scales, Effective Mass, and a Problem with RPA . . . . 31

4 Landau Fermi Liquid Theory and MRPA 33

4.1 Conventional Fermi Liquid Theory . . . . . . . . . . . . . . 35

4.1.1 Fermi Liquid Basics . . . . . . . . . . . . . 35

4.1.2 Fourier Space and Restrictions on Fermi Liquid Coefficients 37 
4.1.3 Boltzmann Transport . . . . . . . . . . . . . 39

4.1.4 Scattering ................. . . . 40

4.1.5 Results of Conventional Fermi Liquid Theory . . . . . . 41

4.2 Landau-Silin Chern-Simons Theory . . . . . . . . . . . 43

4.3 Modified RPA (MRPA) . . . . . . . . . . . . 45

5 Magnetization and $M^{2}$ RPA $\quad 47$

5.1 Zero Frequency Response . . . . . . . . . . . . . . . . . 48

5.2 Binding Magnetization to Composite Fermions . . . . . . . . 50

5.3 Magnetized Modified RPA $\left(\mathrm{M}^{2} \mathrm{RPA}\right)$. . . . . . . . . . . 51

5.4 Fitting into Fermi Liquid Theory . . . . . . . . . . . . . . . 52

5.4.1 Separating Singular Fermi Liquid Coefficients . . . . . . 53

5.4 .2 Relation to $\mathrm{M}^{2} \mathrm{RPA}$.............. 54

6 Perturbative Approaches and Trouble in the Infrared 55

6.1 The Question of a Small Parameter . . . . . . . . . . . 56

6.2 Diagrammatics ................... . . 58

6.3 Infrared Divergences . . . . . . . . . . . . . . . . 64

6.4 Divergent Fermi Liquid Theory . . . . . . . . . . . . . . . . 67

7 Wavefunction Picture of Composite Fermions $\quad 70$

7.1 Wavefunctions and Lowest Landau Level Physics . . . . . . . . 71

7.2 Laughlin's Wavefunction . . . . . . . . . . . . . . . . . 73

7.3 The $\nu=\frac{1}{2}$ Wavefunction . . . . . . . . . . . . . . . . . 74

7.4 Excitations and Small $\Delta B \ldots \ldots$. . . . . . . . . . . 77

7.5 Jain's Wavefunctions . . . . . . . . . . . . . . . . . . 78

7.6 Wavefunctions vs. Chern-Simons Theory . . . . . . . . . . . . 80

7.7 Response of Neutral Dipole Composite Fermions . . . . . . . . 81

7.7.1 Dipole Fermion Variables . . . . . . . . . . . 82

7.7 .2 Response Functions . . . . . . . . . . . . . . 84

7.7.3 K-invariance . . . . . . . . . . . . . . . 85

7.7.4 Dipole RPA . . . . . . . . . . . . . . . 87

8 Selected Experiments $\quad \mathbf{8 9}$

8.1 Surface Acoustic Waves . . . . . . . . . . . . . . . 89

8.2 Coulomb Drag . . . . . . . . . . . . . . . . . 92

8.3 Activation Energy . . . . . . . . . . . . . . . . 94

8.4 Shubnikov-deHaas Oscillations . . . . . . . . . . . . 94

8.5 Geometric Experiments . . . . . . . . . . . . . 95

9 Last Words $\quad 96$ 
A Noninteracting Response Functions in Zero Field

B RPA in yet another language 


\section{Introduction}

Since its discovery, the field of quantum Hall physics ${ }^{1-4}$ has had more than its share of surprising discoveries. Many, if not most, of the important concepts in the field seem so exotic that it is almost certain that they never would have been taken seriously were they not so strongly supported by experiments and exact diagonalizations. The composite fermion is one of these unbelievable concepts.

The field of composite fermion physics began in 1989 with a paper by Jainendra Jain ${ }^{5,6}$ who pointed out that there is a mapping between the wavefunctions of integer quantized Hall states and approximate - but extremely good - wavefunctions for fractional quantized Hall states. This wavefunction mapping can be thought of as binding an even number of vortices (zeros) of the wavefunction to each electron, turning it into a "composite" fermion.

Although the idea of the composite fermion initially met with a fair amount of resistance from the theoretical community, slowly its validity was accepted and the idea was further developed by several groups. Using machinery developed earlier in the bosonic picture of the fractional quantum Hall effect ${ }^{7-9}$ and in work on anyon superconductivity ${ }^{10-12}$, a field theoretical formalism was developed that roughly corresponded to Jain's composite fermion wavefunction approach ${ }^{13-15}$. In this "Chern-Simons" approach, the electron is exactly modeled as as fermion bound to an even number of fictitious flux quanta which in some sense represent the even number of vortices of Jain's composite fermion. The great advantage of this Chern-Simons approach is that it allows for simple yet systematic (or at least semi-systematic) calculations of quantities such as conductivities that are measurable experimentally.

The Chern-Simons fermionic field theory was first used by Lopez and Frad$\operatorname{kin}^{13}$ to describe the Jain series of incompressible fractional quantized Hall states. Shortly thereafter, it was used by Halperin, Lee, and Read (known as HLR $)^{14}$ and Kalmeyer and Zhang ${ }^{15}$ to describe the even denominator states. The most surprising result of this approach is that the even denominator states are compressible and are Fermi-liquid-like.

In this chapter, I will review recent work on the Chern-Simons fermion description of fractional Hall states at a level that should be accessible to readers who are not experts in the field. I hope that this chapter will serve, at least partially, as an introduction to the other chapters in this book. Since the field of composite fermion physics is quite large, no single article could hope to discuss all of the work that has been done in the field ${ }^{a}$. I have thus chosen to discuss some subset of topics that fit together to form a coherent

\footnotetext{
${ }^{a}$ This entire book does not even come close to discussing all of the work done in this field.
} 
story, and hopefully I will touch on many of the more important issues that have been raised in the last few years. However, many other important works will certainly be neglected and I will apologize in advance for these omissions.

The outline of this chapter is as follows. Section 2 is an introduction to the Chern-Simons theory. We begin with a review of integer quantized Hall effect and move on to the Chern-Simons theory and the Chern-Simons mean field approximation. Within this approximation, we will discuss both the incompressible fractional quantized Hall states as well as the compressible Fermiliquid-like even denominator states. The mean field, of course, is extremely crude and in particular does not correctly predict response function such as the Hall conductivity. To fix this problem, in section 3 we will discuss the more sophisticated Chern-Simons RPA approximation in great depth. Although the RPA repairs some of the problems of mean field theory, it cannot be made to give the correct energy scale for low energy excitations while maintaining Galilean invariance. Furthermore, as we will see in section 6 , attempts to systematically calculate corrections to RPA are plagued with infrared divergences of quantities such as the effective mass.

The problems with the RPA description encourage us to turn to a phenomenological Landau-Fermi liquid theory approach in section 4 . We begin by giving a detailed review of the Landau description and describe how we expect the Chern-Simons fermi liquid to fit into this picture. We then develop the $\mathrm{MRPA}^{16}$, a phenomenological approximation motivated by Fermi liquid theory that repairs the RPA's problems with energy scales. We realize that even this improved approximation does not properly represent so-called magnetization effects, requiring us to propose yet another approximation ${ }^{17,18}$, the $\mathrm{M}^{2} \mathrm{RPA}$ and show how this approximation can also fit into the Landau picture.

In section 6 we attempt a more systematic perturbation expansion and wrestle with the pathologies of this Chern-Simons theory. In particular, we are concerned with to what extent the Landau fermi liquid picture we developed above is consistent with the results of perturbative calculations.

In section 7 we discuss the wavefunction approach to composite fermions, which leads us to a somewhat different picture of neutral dipole fermions at even denominator filling fractions (compared to the Chern-Simons fermions which are charged), and we relate this neutral dipole picture to the Chern-Simons picture. Finally, in section 8 we will critically discuss some of the experimental results, and in section 9 we will briefly mention some other directions and summarize what we have learned. 


\section{Introduction to Chern-Simons Fermions}

In this section background material will be given in detail. Readers who desire a more thorough review of previous works in quantum Hall physics are referred to References $1-4$.

\subsection{Quantum Hall Effect Basics}

This section is written for the reader who needs to be reminded of a few of the essentials of quantum Hall physics. The experienced reader is encouraged to skip to section 2.2 referring back to this primer only when necessary.

We begin by considering a two dimensional electron gas (2DEG) consisting of $N$ interacting electrons of band mass $m_{\mathrm{b}}$ in a magnetic field $B=\nabla \times \mathbf{A}$ perpendicular to the plane of the system (We will call the normal to the plane the $\hat{\mathbf{z}}$ direction). We will always neglect the spin degree of freedom of the electrons, assuming that the magnetic field is sufficiently high such that the electrons are spin-polarized. This assumption is reasonable for many quantum Hall experiments, and it will make our discussion much simpler.

The Hamiltonian for such a spin-polarized (or spinless) system of electrons is written as

$$
H_{\mathrm{e}}=\sum_{j} \frac{\left[\mathbf{p}_{j}+\frac{e}{c} \mathbf{A}\left(\mathbf{r}_{j}\right)\right]^{2}}{2 m_{\mathrm{b}}}+\sum_{i<j} v\left(\mathbf{r}_{i}-\mathbf{r}_{j}\right) .
$$

where $v$ is the two body interaction potential, $c$ is the speed of light and $-e$ is the charge of the electron ${ }^{a}$. Here, $m_{\mathrm{b}}$ is the bare electron band mass ${ }^{b}$. We will often specialize to the physical case of Coulombic interaction

$$
v(\mathbf{r})=v(|\mathbf{r}|)=\frac{e^{2}}{\epsilon|\mathbf{r}|}
$$

with $\epsilon$ the background dielectric constant. However, it will also be useful at times to consider other forms of electron-electron interaction. It is amusing that so much interesting quantum Hall physics will come from such a simple looking Hamiltonian.

\subsubsection{Integer Quantized Hall Effect : Single Electron Physics}

If we ignore the interactions between electrons (setting $v=0$ ) the Hamiltonian breaks up into the sum of single particle Hamiltonians which can be easily

\footnotetext{
${ }^{a}$ This inconvenient convention is the source of endless sign problems. We have Ben Franklin to blame for this headache.

${ }^{b}$ It is convenient that the subscript b can stand for 'bare' or 'band'
} 
diagonalized (See Ref. 1 for details). We can solve for the eigenfunctions $\varphi_{k n}(\mathbf{r})$ whose eigenenergies are given by $E_{n k}=E_{n}=\hbar \omega_{\mathrm{c}}\left(n+\frac{1}{2}\right)$ where

$$
\omega_{\mathrm{c}}=\frac{e B}{m_{\mathrm{b}} c}
$$

is the cyclotron frequency ${ }^{c}$. For each value of $n$, the index $k$ can take $B / \phi_{0}$ different values per unit area of the system where ${ }^{d}$

$$
\phi_{0}=\frac{2 \pi \hbar c}{e}=2 \pi \quad(\text { in units with } \hbar=e=c=1)
$$

is the quantum mechanical unit of flux. Thus the spectrum breaks up into highly degenerate "Landau bands" whose degeneracy is given by the value of the magnetic field and the area of the system. We can then define a natural magnetic length scale

$$
l_{B}=\sqrt{\frac{\phi_{0}}{2 \pi B}}
$$

such that the filling fraction

$$
\nu=\frac{\phi_{0} n_{\mathrm{e}}}{B}=2 \pi n_{\mathrm{e}} l_{B}^{2}
$$

with $n_{\mathrm{e}}$ the electron density gives the number of Landau levels completely filled. Note that when an integer number of Landau bands are completely filled there is a discontinuity in the chemical potential (i.e., when $\nu$ is an integer, adding the $(n+1)^{s t}$ electron costs $\hbar \omega_{\mathrm{c}}$ more energy than adding the $n^{\text {th }}$ electron). This discontinuity in the chemical potential, or "thermodynamic incompressibility" is the trademark of a quantized Hall state. Another way to describe this incompressibility is to note that when there are an integer number of Landau levels filled there is an energy gap of

$$
E_{g}=\hbar \omega_{\mathrm{c}}=\hbar e B /\left(m_{\mathrm{b}} c\right)
$$

between the ground state and the lowest excited states since any excitation of this system must involve promoting an electron to a higher Landau level.

By using Galilean invariance, one can easily show that a perfectly clean system at finite filling fraction $\nu$ has zero diagonal (longitudinal) DC resistivity

\footnotetext{
${ }^{c}$ The discrete energy levels can be understood semiclassically as being the Bohr quantization of the electron making cyclotron orbits in a magnetic field. The index $k$ corresponds to the degeneracy of the many places we can put the center of the cyclotron orbit.

$d$ The reader is warned that $\phi_{0}=2 \pi$ is used as often as not in the literature, and factors of $\hbar, c$ and $e$ tend to appear and disappear almost randomly.
} 
$\rho_{x x}$ and a DC Hall resistivity given by $\rho_{x y}=\frac{1}{\nu} \frac{h}{e^{2}}$ with $h$ Planck's constant and $e$ the electron charge $^{e}$. A general theorem ${ }^{1}$ then states that when a perfectly clean system forms an incompressible state at some filling fraction $\nu_{0}$ (an integer for example), then a system with small but nonzero disorder will display a quantized Hall state for some range of filling fractions around $\nu_{0}$, with zero diagonal resistivity (despite the disorder) and quantized Hall resistivity. The DC resistivity matrix of this quantized Hall state for a range of fillings around filling fraction $\nu_{0}$ is thus given by ${ }^{f}$

$$
\rho=\frac{h}{e^{2}}\left[\begin{array}{cc}
0 & 1 / \nu_{0} \\
-1 / \nu_{0} & 0
\end{array}\right] \text {. }
$$

where the resistivity matrix is defined to relate the local current density to the electric field via

$$
\mathbf{j}=\rho \mathbf{E} .
$$

It has been shown that gauge invariance guarantees the precise quantization of the resistance ${ }^{g}$ of the quantized Hall state ${ }^{19}$. Indeed, in Von Klitzing's now famous paper first demonstrating the integer quantized Hall effect ${ }^{20}$, it was proposed that this effect could be used for precision measurements of the resistance quantum $h / e^{2}$. Such measurements have now been performed to a precision of a part in $10^{9}$ and are now used as an international metrological standard $^{21}$. This incredible precision is roughly analogous to measuring the circumference of the earth to within a single centimeter ${ }^{h}$. Fundamentally, this

\footnotetext{
${ }^{e}$ To show this, consider applying an electric field $\mathbf{E}$ to the system. In a reference frame moving at a velocity $\mathbf{v}$, the electric field is $\mathbf{E}-\frac{1}{c} \mathbf{v} \times \mathbf{B}$. Choosing $\mathbf{v}$ appropriately (such that the Lorentz force $\mathbf{F}=e \mathbf{E}+\frac{e}{c} \mathbf{v} \times \mathbf{B}$ vanishes) then in this new frame, we simply have a system of electrons in magnetic field $\mathbf{B}$ but in zero electric field so there is no net current in this frame. The current in the original frame is then just the boost velocity times the charge density of the system. Thus, we find that $\mathbf{j}=\hat{\mathbf{z}} \times \mathbf{E}\left(n_{\mathrm{e}} e c / B\right)$ or $\rho_{x y}=n_{\mathrm{e}} e c / B=h /\left(e^{2} \nu\right)$ and $\rho_{x x}=0$. Clearly, any amount of disorder will ruin this argument.

${ }^{f}$ Note that the inverse of this matrix (the conductivity matrix) also has zero diagonal components. Thus, we have the interesting case of having zero longitudinal resistivity as well as zero longitudinal conductivity. This, of course, is just the statement that the current runs precisely perpendicular to the voltage.

${ }^{g}$ For most systems there is an important distinction between the resistivity (in this case a matrix) which relates local currents to local electric fields and the various possible resistances of the system which relate the voltage measured between two contacts to the currents passing through two leads. However, for quantized Hall states, due to the zero longitudinal resistivity, it can easily be shown ${ }^{1}$ that any such resistance measurement (ratio of a current to a voltage) must either be zero (for any longitudinal measurement) or must be equal to the quantized Hall value of the resistivity $\frac{1}{\nu} h / e^{2}$ (for any Hall measurement).

${ }^{h}$ Some atomic physics experiments have achieved precisions of a part in $10^{14}$ or even better. However, when one recalls that the quantum Hall system is full of all sorts of impurities and other garbage, even atomic physicists are impressed.
} 
precise quantization is based on the incompressibility, or rigidity, of the state. When the clean system is incompressible, small changes in filling fraction result in defects in the state (quasiparticles), rather than a global destruction of the state. So long as these defects in the state become localized due to disorder they do not ruin the macroscopic integrity of the quantum Hall state, such that for a range of filling fractions around the quantized value, the resistance of the state remains unchanged. This situation is quite reminiscent of the situation in a type II superconductor ${ }^{9}$ where applying a magnetic field creates a vortex; and so long as the vortex remains pinned, the system remains superconductive.

\subsubsection{Fractional Quantized Hall Effect : Interactions}

In the single electron picture at filling fractions that are not integers, there is an enormous degeneracy of states associated with the Landau level degeneracy. Consider for example, the filling fraction $\nu=\frac{1}{3}$. Here, we have a macroscopic number $N$ of electrons and $3 N$ states in the lowest Landau level. Thus, there are $(3 N) ! /(N !(2 N) !)$ ways to distribute the electrons which is an immensely huge number. Neglecting interactions, all of these ways have precisely the same energy $\left(\frac{N}{2} \hbar \omega_{\mathrm{c}}\right)$. Of course, when one includes interactions, some of these ways of distributing the electrons will be found to be more favorable than other ways. However, owing to the enormous degeneracy of states, one might naively guess that the state at filling fraction $\nu=\frac{1}{3}$ would be quite compressible because of the high density of states all with very similar energies. It thus came as quite a surprise when it was first discovered ${ }^{22}$ that incompressible "fractional" quantized Hall states can form at many filling fractions ( $\operatorname{such}$ as $\nu=\frac{1}{3}$ ) that are not integers. The existence of these fractional Hall states tells us that somehow, interactions are causing incompressible states to be formed at these non-integer filling fractions. In order to understand fractional Hall states, we will have to find a way to treat inter-electron interactions as well as the kinetic energy of the Landau levels. This, however, is a difficult challenge.

To begin to address this challenge we should carefully consider the two terms in the Hamiltonian (Eq. 1). Since the kinetic term of the Hamiltonian is easily diagonalized, one might consider treating the interaction term in some sort of perturbation theory. However, the rules of degenerate perturbation theory require us to diagonalize each degenerate subspace first before proceeding. In our case, the degenerate subspace of a partially filled Landau level is immensely huge and this first step is almost as impossible as solving the entire problem, thus rendering such a perturbative approach hopeless. The interaction term in the Hamiltonian, on the other hand, if treated alone, results in a Wigner crystal which is quite a different state from the fractional Hall state. 
It is only the interplay between the kinetic and potential terms that yields the fractional quantized Hall effect. Thus, it seems that the traditional systematic perturbative approaches for understanding the fractional quantum Hall effect are out of the question.

Indeed, much of our understanding of fractional Hall effect is based on understanding the properties of trial wavefunctions, and not on any systematic

perturbation approach ${ }^{23,6}$. Only recently the Chern-Simons field theories ${ }^{9,13,14}$ have provided a starting point for a semi-systematic perturbative approach to understanding these states. As we will see below, these approaches have their share of complications (hence the prefix "semi" before the word "systematic"). Nonetheless, the Chern-Simons field theories have led to a much deeper understanding of many aspects of the fractional quantum Hall effect. Most of the remainder of this paper will be devoted to elucidating aspects of the so-called "Chern-Simons-Fermionic" description of the fractional quantum Hall regime.

\subsection{Chern-Simons Transformation}

The Chern-Simons approach is employed by making a transformation on the phase of the many-electron wavefunction. Writing the electron wavefunction as $\Psi_{\mathrm{e}}\left(\mathbf{r}_{1}, \mathbf{r}_{2}, \ldots \mathbf{r}_{N}\right)$ with $\mathbf{r}_{j}$ the position of the $j^{\text {th }}$ electron, we define a new transformed wavefunction

$$
\Phi\left(\mathbf{r}_{1}, \mathbf{r}_{2}, \ldots, \mathbf{r}_{N}\right)=\left[\prod_{i<j} e^{-i \tilde{\phi} \theta\left(\mathbf{r}_{i}-\mathbf{r}_{j}\right)}\right] \Psi_{\mathrm{e}}\left(\mathbf{r}_{1}, \mathbf{r}_{2}, \ldots, \mathbf{r}_{N}\right)
$$

where

$$
\tilde{\phi}=2 m
$$

is an even integer, and the 'function' $\theta\left(\mathbf{r}_{i}-\mathbf{r}_{j}\right)$ is the angle formed by the vector $\mathbf{r}_{i}-\mathbf{r}_{j}$ with the $\hat{\mathbf{x}}$ axis. Note that the 'function' $\theta$ is defined only modulo $2 \pi$, but becomes well defined once exponentiated (one should consider it to be multiple valued rather than to have branch cuts).

It is then easy to see that ${ }^{13,14}$ if $\Psi_{\mathrm{e}}$ is a solution of the Schroedinger equation $H_{\mathrm{e}} \Psi_{\mathrm{e}}=E \Psi_{\mathrm{e}}$, then $\Phi$ is a solution to the Schroedinger equation $H \Phi=E \Phi$ with

$$
H=\sum_{j} \frac{\left[\mathbf{p}_{j}+\frac{e}{c} \mathbf{A}\left(\mathbf{r}_{j}\right)-\frac{e}{c} \mathbf{a}\left(\mathbf{r}_{j}\right)\right]^{2}}{2 m_{\mathrm{b}}}+\sum_{i<j} v\left(\mathbf{r}_{i}-\mathbf{r}_{j}\right)
$$


where $\mathbf{a}$ is the "Chern-Simons" vector potential

$$
\mathbf{a}\left(\mathbf{r}_{i}\right)=i \nabla_{i}\left[\prod_{j} e^{-i \tilde{\phi} \theta\left(\mathbf{r}_{i}-\mathbf{r}_{j}\right)}\right]=\frac{\tilde{\phi} \phi_{0}}{2 \pi} \sum_{j=1}^{N} \frac{\hat{\mathbf{z}} \times\left(\mathbf{r}_{i}-\mathbf{r}_{j}\right)}{\left|\mathbf{r}_{i}-\mathbf{r}_{j}\right|^{2}}
$$

and $\tilde{\phi}=2 m$.

Since $\mathbf{a}$ is a gradient, $\nabla \times \mathbf{a}(\mathbf{r})=0$ for all $\mathbf{r}$ not equal to the position of one of the electrons. Thus we might think of this transformation as being just a gauge transformation. However, due to the nonsinglevaluedness of the function $\theta$, the gauge transformation is singular and we have a singularity, $\nabla \times \mathbf{a}(\mathbf{r})=\tilde{\phi} \phi_{0} \delta\left(\mathbf{r}-\mathbf{r}_{j}\right)$ at the position of each electron. The Chern-Simons magnetic field $b(\mathbf{r})$ associated with the vector potential $\mathbf{a}$ is then given by

$$
b(\mathbf{r})=\nabla \times \mathbf{a}(\mathbf{r})=\phi_{0} \tilde{\phi} n(\mathbf{r})=2 \pi \tilde{\phi} n(\mathbf{r})
$$

where $n(\mathbf{r})=\sum_{j} \delta\left(\mathbf{r}-\mathbf{r}_{j}\right)$ is the local particle density. Note that since the magnitude of the wavefunction is not changed by this Chern-Simons transformation, the electron density and the transformed fermion density are equal, as can easily be seen by writing

$$
\begin{aligned}
n_{\mathrm{e}}\left(\mathbf{r}_{1}\right) & =\int d \mathbf{r}_{2} \int d \mathbf{r}_{3} \cdots \int d \mathbf{r}_{N}\left|\Phi\left(\mathbf{r}_{1}, \mathbf{r}_{2}, \ldots, \mathbf{r}_{N}\right)\right|^{2} \\
& =\int d \mathbf{r}_{2} \int d \mathbf{r}_{3} \cdots \int d \mathbf{r}_{N}\left|\Psi\left(\mathbf{r}_{1}, \mathbf{r}_{2}, \ldots, \mathbf{r}_{N}\right)\right|^{2}=n_{\mathrm{f}}\left(\mathbf{r}_{1}\right) .
\end{aligned}
$$

It is also easy to see that if $\Psi$ obeys fermionic statistics in the sense that the wavefunction is antisymmetric under exchange of any two particles, then the transformed wavefunction $\Phi$ is similarly antisymmetric and hence represents a fermionic wavefunction. Thus we should think of the Hamiltonian $H$ as being the Hamiltonian for $N$ interacting transformed fermions. For $\tilde{\phi}$ not equal to an even integer, the resulting wavefunction $\Phi$ would not be antisymmetric. In this case, the resulting Hamiltonian represents particles of non-fermionic statistics — bosonic for $\tilde{\phi}$ an odd integer and anyonic ${ }^{12}$ for non-integer values. Indeed this type of Chern-Simons transformation had been used previously to develop a bosonic description of Quantum Hall states ${ }^{7-9}$, and in the description of anyon superconductors ${ }^{10-12}$.

In summary, the Chern-Simons transformation can be described as the exact modeling of an electron as a fermion attached to $\tilde{\phi}=2 m$ flux quanta. We call these fermions "singularly gauge transformed", "Chern-Simons", or "composite" fermions. ${ }^{i}$ We emphasize that this fictitious Chern-Simons magnetic

${ }^{i}$ The loose use of nomenclature here is somewhat unfortunate. Jain ${ }^{5}$ originally used the 
field (which in truth is just a mathematical convenience) certainly does not exist outside of the two dimensional electron system.

At this point, one might note that we have transformed a relatively simple looking system (electrons in a strong magnetic field interacting via the Coulomb interaction) into a much more complicated looking system (transformed fermions in a strong magnetic field interacting via the Coulomb interaction and via a Chern-Simons gauge field). However, as we will see below, this transformation is actually very useful since the simple looking electron system is actually totally intractable (due to the huge degeneracy of states), whereas the more complicated looking transformed fermion system is actually something we will be able to work with.

\subsection{Mean Field Theory}

The simplest approach to analyzing this transformed Chern-Simons fermion system is to make the mean field approximation in which density is assumed uniform and the Chern-Simons flux quanta attached to the fermions are smeared out into a uniform magnetic field of magnitude

$$
\langle b\rangle=n_{\mathrm{e}} \tilde{\phi} \phi_{0}=2 \pi n_{\mathrm{e}} \tilde{\phi}
$$

with $n_{\mathrm{e}}$ the average density, and $\tilde{\phi}=2 m$ the even number of flux quanta attached to each fermion. Choosing the Chern-Simons flux to be in the opposite direction as the applied magnetic field, this field $\langle b\rangle$ cancels off part of the external magnetic field $B$ leaving a (mean) residual field seen by the transformed fermions

$$
\Delta B=B-\langle b\rangle=B-n_{\mathrm{e}} \tilde{\phi} \phi_{0}
$$

Making this approximation of uniform density or "mean field", the Hamiltonian (Eq. 12) is approximated as

$$
H_{\text {mean-field }}=\sum_{j} \frac{\left[\mathbf{p}_{j}+\frac{e}{c} \boldsymbol{\Delta} \mathbf{A}\left(\mathbf{r}_{j}\right)\right]^{2}}{2 m_{\mathrm{b}}}
$$

where $\boldsymbol{\Delta} \mathbf{A}$ is the vector potential associated with the mean magnetic field $\Delta B$ (i.e., $\nabla \times \boldsymbol{\Delta} \mathbf{A}=\Delta B$ ), which simply describes free fermions in a uniform

term 'composite fermion' to describe a related — but nonetheless distinctly different wavefunction transformation. For a brief period of time, some members of the community made an effort to distinguish between Jain's composite fermions and these Chern-Simonssingularly-gauge-transformed fermions. However, this more specific nomenclature was apparently too clumsy and now the simpler terminology 'composite fermion' is used as often as not to describe the transformed fermions. The relation between Jain's approach and the Chern-Simons approach will be elaborated in section 7 below. 
magnetic field. Note that the Coulomb interaction also disappears at the mean field level where the density is assumed completely uniform throughout the system since it only contributes an (albeit infinite) constant $^{j}$.

\subsubsection{Even Denominator Fractions}

At some special value of the filling fraction, when $B=\langle b\rangle$, the applied magnetic field precisely cancels the Chern-Simons flux at the mean field level (i.e., $\Delta B=$ $0)$. This exact cancellation occurs when $B=\langle b\rangle=\tilde{\phi} \phi_{0} n_{\mathrm{e}}$ or equivalently at the filling fraction

$$
\nu=\frac{n_{\mathrm{e}} \phi_{0}}{B}=\frac{1}{\tilde{\phi}}=\frac{1}{2 m} .
$$

Thus for even denominator filling fractions, at the mean field level we describe the ground state of the system as fermions in zero magnetic field, which is just a filled Fermi sea with Fermi momentum ${ }^{k}$

$$
k_{\mathrm{F}}=\sqrt{4 \pi n_{\mathrm{e}}}=\frac{1}{l_{B} \sqrt{m}} .
$$

The existence of this Fermi-liquid like state at even denominator filling fractions was predicted by Kalmeyer and Zhang ${ }^{15}$ and by Halperin, Lee, and Read $^{14}$. It should be emphasized that it is an extremely surprising result that one can add a huge magnetic field to a system and end up with an effective system that behaves in some ways as if it were in zero magnetic field.

If we are at a magnetic field such that we are close to (but not exactly at) such an even denominator filling fraction, then the applied magnetic field and the Chern-Simons flux do not quite exactly cancel and $\Delta B$ is nonzero. Thus, we have a Fermi sea in a small magnetic field in which case the elementary quasiparticle excitations above the Fermi sea travel in large cyclotron orbits of radius $^{l}$

$$
R_{\mathrm{c}}^{*}=\frac{\hbar c k_{\mathrm{F}}}{e \Delta B}
$$

\footnotetext{
${ }^{j}$ We have similarly assumed that no equilibrium currents are flowing in the system at mean field level. As we will see below in section 3.1, such currents would induce a ChernSimons electric field that would then have to be included too. Such equilibrium currents are important when one thinks about systems with nonuniform density ${ }^{94,17}$.

${ }^{k}$ This value of the Fermi momentum differs by a factor of $\sqrt{2}$ from the Fermi momentum of the electron gas in zero magnetic field since in zero field there two spin states whereas here we have assumed fully polarized spins.

${ }^{l}$ For general circular motion $R=v^{2} m / F$. In a magnetic field, the Lorentz force is $F=e B v / c$. Using $v=\hbar k_{\mathrm{F}} / m$, we find that all factors of the mass $m$ cancel from this expression.
} 
This new length scale has been clearly observed experimentally ${ }^{24}$ giving strong support to the composite fermion picture (See sections 8.1 and 8.5 below for discussion of the relevant experiments).

Although the mean field description gives one a starting point for understanding the physics of the putative composite fermion Fermi liquid, it is clear that mean field theory is quite crude. For example, at the mean field level, since the system is described as being in zero effective magnetic field, one would predict that the Hall conductivity of the system is zero - which is clearly absurd for a system in extremely high magnetic field. Thus we must think about treating our Chern-Simons Hamiltonian (Eq. 12) in an approximation beyond mean field. It should be noted, however, that this mean field description of the even denominator $\nu=\frac{1}{2 m}$ states is a non-degenerate starting point for attempting a controlled perturbation theory — unlike the original highly degenerate partially filled Landau level. In section 3 below, we will begin the discussion of approximations that go beyond this mean field description.

\subsubsection{Jain Series of Fractional Hall States}

For completeness, we also consider the case when the filling fraction is further away from $\nu=\frac{1}{2 m}$. Here, after canceling the mean Chern-Simons field $\langle b\rangle$ with the external field $B$, there is some residual field $\Delta B=B-\langle b\rangle$ left over which, in general, can itself be large. The mean field system is described as noninteracting fermions in the uniform nonzero field $\Delta B$. Fortunately, such a noninteracting system is completely soluble (see section 2.1.1 above). The effective filling fraction $p$ for these gauge transformed fermions is given by (compare with Eq. 6)

$$
p=\frac{n_{\mathrm{e}} \phi_{0}}{\Delta B}
$$

(Note that $p$ can be negative corresponding to a negative $\Delta B$ or a situation where $\langle b\rangle>B$ ). When $p$ is a small integer, at the mean field level, this is just a system of $|p|$ filled Landau levels of fermions, and one should observe the integer quantized Hall effect of transformed fermions. Using Eq. 17 as well as the definition of the filling fraction (Eq. 6), this condition (Eq. 22 with $p$ an integer) yields precisely the Jain series ${ }^{5,6}$ of fractional quantized Hall states

$$
\nu=\frac{p}{2 m p+1 .}
$$

Thus, the fractional quantized Hall effect at these filling fractions is identified with an integer quantized Hall effect of gauge transformed fermions ${ }^{6,13,14}$. The most striking early success of the composite fermion theory was simply that 
this Jain series (Eq. 23) correctly predicts precisely those fractional quantized Hall states in the lowest Landau level that are seen experimentally in roughly the correct order of stability (the most stable states having small $|p|$ and small $|m|){ }^{m}$

In mean field theory the excitation gaps for these fractional quantized Hall states are naturally given by the corresponding effective cyclotron frequency of the composite fermions (analogous to Eq. 7)

$$
E_{\text {gap }}=\hbar \Delta \omega_{\mathrm{c}}^{*}=\frac{\hbar e \Delta B}{m_{\text {gap }}^{*}(\nu) c}
$$

where $m_{\mathrm{gap}}^{*}(\nu)$ is an effective mass of the transformed fermion. There has been increasing experimental evidence ${ }^{24}$ that the fractional Hall gaps do indeed increase (at least roughly) linearly with $\Delta B$ (See section 8.3 below for discussion of the relevant experiments).

At the mean field level (See Eq. 18) we have $m_{\text {gap }}^{*}=m_{\mathrm{b}}$, the bare mass of the electron. However, being that the fractional Hall gap must be set by the inter-electron interaction strength, we realize that the mean field result is not accurate and we should expect the value of $m_{\text {gap }}^{*}$ to be highly renormalized. In section 3.9 below, we will more thoroughly discuss the energy scales in the problem and the expected value of the effective mass. Unfortunately, we will find that this discrepancy is not just a problem in mean field - it will persist even in the more sophisticated RPA calculation. Furthermore, in section 6.3 below we will find that systematic attempts to actually calculate the effective mass are plagued with infrared divergences.

Another problem with the mean field theory is that the quantized Hall resistivity is not correctly obtained. Since the $\nu=\frac{p}{2 m p+1}$ state is just an integer $\nu=p$ state of transformed fermions, we have at the mean field level that the Hall resistivity is given by $\rho_{x y}=h /\left(p e^{2}\right)$ rather than the correct quantized value of $\rho_{x y}=h /\left(\nu e^{2}\right)=(2 m p+1) h /\left(p e^{2}\right)$ which we know we should obtain by Galilean invariance (see section 2.1.1 above). Similarly, in mean field theory the finite frequency response will be incorrectly predicted as well (as we will see in section 3.8 below). Fortunately, some of these problems with mean field response functions are corrected in more sophisticated theories such as RPA that we will consider next.

Despite these shortcoming, however, the Chern-Simons mean field description is quite appealing due to its impressive simplicity. Furthermore, as men-

\footnotetext{
${ }^{m}$ In very high quality samples at low temperature, additional fractional Hall states are seen that do not fit this formula. Many of the strongest of these states $(\operatorname{such}$ as $\nu=4 / 5)$ can be simply described as the particle-hole conjugate of simple state (in this case $4 / 5=1-1 / 5$ ). Others observed states are thought to be spin unpolarized states. Other states may require a hierarchical construction even within the composite fermion framework ${ }^{6}$.
} 
tioned above, the mean field theory is a nondegenerate state around which to attempt a controlled (or semi-controlled) perturbation theory or to calculate systematic (or semi-systematic) corrections.

\section{RPA}

One of the great appeals of the Chern-Simons Fermion theory is that it allows for the analytic calculation of physically relevant quantities. Indeed, an argument can be made that a theory is only useful if one can use it to make predictions for physical quantities. In section 2 above we used mean field theory to make an number of nontrivial predictions (scaling of gaps, effective cyclotron radius, existence of certain types of states at certain filling fractions, etc.). However as we saw above, mean field - being the simplest possible approximation - also gets a number of important physical results wrong.

At the mean field level, one considers the density (and current) of the system to be everywhere constant. When the density is everywhere constant, the Coulomb interaction has no effect on the response of the system (contributing just an infinite constant to the energy of the system). Similarly at mean field level, the effect of the bound Chern-Simons flux is just to impose a constant effective magnetic field on the system. When we go beyond mean field level, we must treat fluctuations in densities and currents and find that the Coulomb and Chern-Simons terms have much more nontrivial effects. For example individual fermions should certainly interact via the Coulomb interaction such that when there is a local density fluctuation in one place, the nearby fermions feel the resulting Coulomb potential and respond to it. Fortunately, however, so long as we confine our attention to calculations of resistivities and conductivities (and not the full electromagnetic response), we will find that this Coulomb term will not be too important. Similarly, and more importantly, the bound Chern-Simons flux also causes an interaction - as a fermion moves, it carries its flux quanta with it and the other fermions feel the resulting change in the Chern-Simons vector potential. Proper treatment of this Chern-Simons interaction is required to obtain the proper Hall conductivity for the composite fermion system.

The simplest approximation beyond mean field is the RPA or Random Phase Approximation ${ }^{a}$. The RPA for Chern-Simons theories was first developed in the context of anyon superconductivity ${ }^{10-12}$. A similar approximation was then used by Lopez and Fradkin ${ }^{13}$ (See also the Chapter by Lopez and Fradkin in this book) to study the Jain series of fractional Hall states. Most

\footnotetext{
${ }^{a}$ The RPA is also sometimes given the more descriptive name of "Time Dependent Hartree" approximation. The name 'Random Phase' is almost a historical accident.
} 
recently, the RPA was exploited by Kalmeyer and Zhang ${ }^{15}$ and extensively by Halperin, Lee, and $\operatorname{Read}^{14}$ for the study the even denominator states ${ }^{b}$.

\subsection{Chern-Simons RPA Basics}

Below, in sections 3.2-3.6, we will give a detailed description of the RPA, along with more formal definitions of various useful response functions. Here, we will give a much rougher description that, although less formal, will be sufficient for many of our needs.

In the Chern-Simons theory, an excess density $\delta n$ carries an excess ChernSimons flux of $\tilde{\phi}=2 m$ flux quanta per particle resulting in an excess ChernSimons magnetic field

$$
\delta b=\phi_{0} \tilde{\phi} \delta n=2 \pi \tilde{\phi} \delta n .
$$

Similarly, a composite fermions' current $\mathbf{j}$ carries a current of flux tubes, thus inducing a Chern-Simons electric field given by

$$
\mathbf{e}=\phi_{0} \tilde{\phi} \frac{e}{c}(\hat{\mathbf{z}} \times \mathbf{j})=2 \pi \tilde{\phi}(\hat{\mathbf{z}} \times \mathbf{j})
$$

To see where this electric field comes from, we consider a system with a current $\mathbf{j}$ running in it locally. In a frame moving with velocity $\mathbf{v}=\mathbf{j} / n_{\mathrm{e}}$, the particles are stationary and there is only a Chern-Simons magnetic field. When we boost back to the laboratory frame, some of the magnetic field is transformed into this Chern-Simons electric field ${ }^{c}$. We might also have guessed this result using Faraday's law by considering a closed loop and moving a current of flux tubes across the loop - the EMF being proportional to and perpendicular to the current crossing across the loop. It should be noted that this ChernSimons field - like the Chern-Simons flux - is fictitious in the sense that it is not measurable by a voltmeter - only the other transformed fermions in the 2DEG will see this field.

We now declare that the Chern-Simons fermions have some conductivity matrix $\sigma_{\mathrm{CF}}$ (the so-called "composite fermion conductivity"), but that they should respond, not only to the physical electric field $\mathbf{E}$ (that measured by a voltmeter), but also to the self-consistently induced Chern-Simons electric field e. Thus, we should have

$$
\mathbf{j}=\sigma_{\mathrm{CF}}(\mathbf{E}+\mathbf{e}(\mathbf{j})) .
$$

\footnotetext{
${ }^{b}$ It should be noted that the form of the RPA used in Refs. 10-12 appear somewhat different from the form used by $\mathrm{HLR}^{14}$ (which is what we follow here). In appendix B we will show that these prescriptions are equivalent.

${ }^{c} \mathrm{My}$ appreciation to Ady Stern for pointing out this clear argument. See also section 6.3 below for another derivation of this interaction through the Lagrangian approach. See also Ref. 13.
} 
We now rewrite Eq. 26 as

$$
\mathbf{e}=-\rho_{\mathrm{CS}} \mathbf{j}
$$

with

$$
\rho_{\mathrm{CS}}=\frac{2 \pi \hbar \tilde{\phi}}{e^{2}}\left[\begin{array}{cc}
0 & 1 \\
-1 & 0
\end{array}\right]
$$

such that we can convert Eq. 27 to an expression for the resistivity matrix (defined by $\mathbf{E}=\rho \mathbf{j}$ ) given by

$$
\rho=\rho_{\mathrm{CF}}+\rho_{\mathrm{CS}}
$$

where $\rho_{\mathrm{CF}}=\left[\sigma_{\mathrm{CF}}\right]^{-1}$ is the "composite fermion resistivity" matrix.

One quantity of particular interest is the longitudinal conductivity $\sigma_{x x}$ of the electrons. This is obtained by inverting the matrix $\rho$ to yield, $\sigma_{x x}=$ $\rho_{y y}^{\mathrm{CF}} / \operatorname{Det}\left[\rho^{\mathrm{CF}}+\rho^{\mathrm{CS}}\right]$. For a typical experimental system, near the even denominator state, $\nu=\frac{1}{\tilde{\phi}}$, the $\rho^{\mathrm{CS}}$ dominates the denominator giving

$$
\sigma_{x x} \approx \rho_{y y}^{\mathrm{CF}}\left(\frac{e^{2}}{\tilde{\phi} h}\right)^{2}
$$

Thus far, all of these manipulations might be thought of as a complicated way to define the composite fermion conductivity $\sigma_{\mathrm{CF}}$. However, in terms of this quantity, the Chern-Simons RPA can be given a very simple definition. Here, the RPA approximation is simply the statement that the composite fermion conductivity should be approximated as the mean field conductivity (i.e., that of a system of noninteracting fermions of mass $m_{\mathrm{b}}$ in magnetic field $\Delta B$ - see Eq. 18). Thus, at RPA level, we think of the composite fermions as being free fermions that respond to the total electric field - including both the physical electric field and the self-consistently induced Chern-Simons field.

As an example, we consider the DC response of a system of electrons at filling fraction $\nu=\frac{p}{2 m p+1}$. We make the composite fermion transformation and map this problem to a system of fermions at filling fraction $p$ which displays the integer quantized Hall effect. As mentioned above in section 2.3.2, the mean field value of this resistivity $\left(\rho_{x y}=\frac{1}{p} h / e^{2}\right)$ is not the correct value for the fractionally quantized $\nu=\frac{p}{2 m p+1}$ state. However, using the RPA

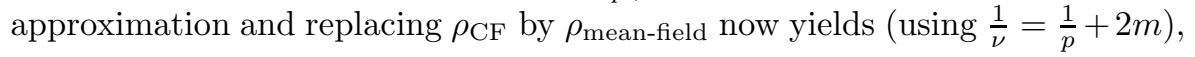

$$
\begin{aligned}
\rho & =\rho_{\text {mean-field }}+\rho_{\mathrm{CS}} \\
& =\frac{h}{e^{2}}\left[\begin{array}{cc}
0 & 1 / p \\
-1 / p & 0
\end{array}\right]+\frac{h}{e^{2}}\left[\begin{array}{cc}
0 & 2 m \\
-2 m & 0
\end{array}\right]=\frac{h}{e^{2}}\left[\begin{array}{cc}
0 & 1 / \nu \\
-1 / \nu & 0
\end{array}\right]
\end{aligned}
$$


which is the correct quantized Hall resistivity for the state. Thus, at the RPA level (but not at mean field level) we recover the fundamental thermodynamical quantity of the quantized Hall resistance.

On a physical level, the way the Hall resistance occurs is the following. We run a current through the sample which carries the Chern-Simons flux quanta through the system creating a Chern-Simons electric field (by the Faraday effect). The other fermions see this electric field, and in order for them to continue to move straight there must be no net electric field, so there must be a physical electric field built up to cancel this Chern-Simons field.

We note that using this approach, we could similarly calculate any finite frequency or finite wavevector response by simply inserting the finite frequency mean field (noninteracting) resistivity into Eq. 30. Finite frequency and wavevector conductivities can be calculated explicitly for noninteracting systems and are discussed further below. A convenient approximate calculation of these conductivities can be made using the Boltzmann approach, which will be discussed in section 4 .

As another example, at finite $q$, and low frequency, $\rho_{y y}$ for a noninteracting system in zero magnetic is given roughly by (this result is from section 4 below where we obtain the result Eq. 104 which yields the following when expanded for small $\Omega$ and $F_{1}=0$ )

$$
\begin{aligned}
\rho_{y y}(q) & =\frac{q}{k_{\mathrm{F}}} \frac{h}{e^{2}} \quad \text { for } \quad q \gg 2 / l \\
& =\frac{2}{k_{\mathrm{F}}} \frac{h}{e^{2}} \quad \text { for } \quad q<2 / l
\end{aligned}
$$

where $l=v_{\mathrm{F}}^{*} \tau$ is some disorder scattering length. Using these expressions as $\rho^{\mathrm{CF}}$, we would then predict that at $\nu=\frac{1}{2 m}$, the low frequency conductivity is given by (using Eq. 31)

$$
\begin{aligned}
\sigma_{x x}(q) & \approx \frac{e^{2}}{h} \frac{q}{\tilde{\phi}^{2} k_{\mathrm{F}}} \text { for } q \gg 2 / l \\
& \approx \frac{e^{2}}{h} \frac{2}{k_{\mathrm{F}} l \tilde{\phi}^{2}} \quad \text { for } \quad q<2 / l
\end{aligned}
$$

This enhanced finite wavevector conductivity linear in $q$ has indeed been observed experimentally ${ }^{24}$ (See section 8.1 below) for large $q$.

The astute reader might notice that in our above discussion of the RPA, we did not ever mention the effects of the Coulomb interaction. Fortunately, so long as we confine our attention to calculations of resistivities and conductivities we will find that this Coulomb term will not be too important. However, in 
calculations of the full electromagnetic response function (which we will define next) the Coulomb interaction will be important.

\subsection{Electromagnetic Response $K_{\mu \nu}$}

Thus far, we have concentrated on calculating the resistivity, or conductivity tensors. There are, however, many other important response functions that we might consider calculating - the most important of which is known as the electromagnetic response matrix $K$. Below, we will relate this object to a number of more familiar quantities, such as the conductivity of the system.

To define $K$, a weak vector potential $A_{\mu}^{\text {ext }}$ is externally applied to the system inducing a current $j_{\mu}$. Here we define $A_{0}$ to be the scalar potential, and $j_{0}$ to be the induced density modulation $n-n_{\mathrm{e}}$ (with $n_{\mathrm{e}}=\langle n\rangle$ ). In linear response theory, we can define the response kernel $K_{\mu \nu}\left(\mathbf{r}, t ; \mathbf{r}^{\prime}, t^{\prime}\right)$ such that

$$
j_{\mu}(\mathbf{r}, t)=e \int_{-\infty}^{t} d t^{\prime} \int d \mathbf{r}^{\prime} K_{\mu \nu}\left(\mathbf{r}, t ; \mathbf{r}^{\prime}, t^{\prime}\right) A_{\nu}^{\operatorname{ext}}\left(\mathbf{r}^{\prime}, t^{\prime}\right)
$$

where the sum over the repeated index $(\nu)$ is implied. Assuming translational invariance (in time as well as space), we can write $K_{\mu \nu}\left(\mathbf{r}-\mathbf{r}^{\prime}, t-t^{\prime}\right)$, indicating that Eq. 37 is just a convolution which simplifies in Fourier space to

$$
j_{\mu}(\mathbf{q}, \omega)=e K_{\mu \nu}(\mathbf{q}, \omega) A_{\nu}^{\operatorname{ext}}(\mathbf{q}, \omega)
$$

(Again the repeated index is summed). Another way to think about this response matrix is to imagine applying an external potential $A_{\nu}^{\text {ext }}$ to the system at wavevector $\mathbf{q}$ and frequency $\omega$. Then in linear response, a current $j_{\mu}(\mathbf{q}, \omega)$ is induced given by Eq. 38. We will always choose the convention that the $\mathbf{q}$ is parallel to the $\hat{\mathbf{x}}$ axis such that perturbations are proportional to $e^{i \omega t-i q x}$.

Most generally, we will attempt to calculate the full electromagnetic response matrix $K_{\mu \nu}$ where $\mu$ and $\nu$ take the values $0, x, y$. However, we can simplify our life by using current conservation $\nabla \cdot j+\frac{d}{d t} n=0$ or $q j_{x}=\omega j_{0}$ and choosing the gauge $A_{x}=0$. With $j_{x}$ and $A_{x}$ thus determined, we can then treat $K_{\mu \nu}$ as a $2 \times 2$ matrix with indices taking the values 0 or 1 denoting the time $(0)$ or transverse space $(y)$ components respectively. In this notation the current vector $j_{\mu}$ is $\left(j_{0}, j_{y}\right)$, and the vector potential $A_{\mu}$ is $\left(A_{0}, A_{y}\right)$. Note that from here on, we will routinely drop the explicit matrix subscripts $\mu$ and $\nu$ as well as the explicit $q$ and $\omega$ dependences.

Although all of the elements of the response matrix $K_{\mu \nu}$ are genuine physical quantities, the so-called density density response $K_{00}$ (also called $\chi$ ) is perhaps most important for quantum Hall physics because it can often be 
measured experimentally (See section 8.1 below). Theoretically, $K_{00}$ is also an important quantity, being related to the more familiar dynamical structure factor $S(q, \omega)$ via

$$
-\frac{1}{\pi} \operatorname{Im} K_{00}\left(\mathbf{q}, \omega+i 0^{+}\right)=S(\mathbf{q}, \omega)=\sum_{n}|\langle j|\hat{n}(\mathbf{q})| 0\rangle|^{2} \delta\left(E_{j}-E_{0}-\omega\right)
$$

where $|j\rangle$ is the $j^{\text {th }}$ many body eigenstate whose energy is $E_{j},|0\rangle$ is the ground state (with energy $E_{0}$ ), and $\hat{n}(\mathbf{q})$ is the density operator at wavevector $\mathbf{q}$. Thus, $-\frac{1}{\pi} \operatorname{Im} K_{00}=S$ has poles at the frequencies of each excitation mode of the system, and the residue (or weight) of this pole indicates how easy it is to create this excited state from the ground state by applying a finite wavevector perturbation. (Note that $K_{\mu \nu}$ satisfies Kramers-Krönig relations so the imaginary part completely determines the real part).

We will also define $K^{0}$ to be the response function for noninteracting fermions (The ${ }^{0}$ means noninteracting). In appendix $\mathrm{A}$ we show the full result for $K^{0}$ for fermions in zero field. (See also Refs. 10 and 16 for explicit expressions for the response in finite magnetic field.) The actual calculation of $K^{0}$ is described in more detail in section 6.2 below. It is often convenient to use a Boltzmann approach to approximate $K^{0}$. The advantage of this approach (besides its simplicity) is that it is quite easy to approximate the effects of disorder or Fermi liquid interactions at a phenomenological level. This will be discussed in more depth in section 4 below. Using the Boltzmann approach, one directly obtains the noninteracting conductivity $\sigma^{0}$ which can be converted to the response using $K^{0}=T \sigma^{0} T$ with the conversion matrices $T$ given by Eq. 51 below (we will discuss this more in a moment). Such a Boltzmann calculation is performed for the case of fermions in zero field in appendix A.

\subsection{Hartree Part of the Coulomb Interaction: Separating $\Pi^{v}$}

We now turn to consider the effect of the Coulomb interaction. The Coulomb term in the Hamiltonian (compare Eq. 1) is given by

$$
H_{\mathrm{int}}=\sum_{i<j} v\left(\mathbf{r}_{i}-\mathbf{r}_{j}\right)=\frac{1}{2} \int d \mathbf{r}_{1} \int d \mathbf{r}_{2} n\left(\mathbf{r}_{1}\right) n\left(\mathbf{r}_{2}\right) v\left(\mathbf{r}_{1}-\mathbf{r}_{2}\right) .
$$

with $n(\mathbf{r})=\sum_{i} \delta\left(\mathbf{r}-\mathbf{r}_{i}\right)$ the density operator. The Hartree approximation is then written as ${ }^{d}$

$$
H_{\mathrm{int}}=-\int d \mathbf{r} n(\mathbf{r}) e A_{0}^{v-\mathrm{ind}}(\mathbf{r})
$$

\footnotetext{
${ }^{d}$ The reader may notice that a factor of $\frac{1}{2}$ has disappeared. This is a result of taking $\left\langle n\left(\mathbf{r}_{1}\right)\right\rangle n\left(\mathbf{r}_{2}\right)+n\left(\mathbf{r}_{1}\right)\left\langle n\left(\mathbf{r}_{2}\right)\right\rangle$ as Wick's theorem would suggest.
} 


$$
-e A_{0}^{v-\text { ind }}(\mathbf{r})=\int d \mathbf{r}^{\prime}\left\langle n\left(\mathbf{r}^{\prime}\right)\right\rangle v\left(\mathbf{r}-\mathbf{r}^{\prime}\right)
$$

Physically, the Hartree approximation is just the simple statement that any (expectation value of the) electron density in the system will induce a Coulomb potential which the other electrons then respond to. (Exchange interaction, or "Fock terms" are neglected in the Hartree approximation).

In Fourier space, Eq. 42 simplifies to

$$
-e A_{0}^{v-\operatorname{ind}}(\mathbf{q}, \omega)=v(\mathbf{q})\langle n(\mathbf{q}, \omega)\rangle
$$

where $v(q)$ is the Fourier transformed interaction, given by $2 \pi e^{2} /(\epsilon q)$ for the usual Coulomb case. Suppressing wavevector and frequency dependences, we now write this in the $2 \times 2$ matrix notation (for later convenience),

$$
-e A^{v-\text { ind }}=V j
$$

with

$$
V=\left[\begin{array}{cc}
v(q) & 0 \\
0 & 0
\end{array}\right] .
$$

We now define the total-physical vector potential that includes both the external part and that induced by the Coulomb interaction ${ }^{e}$

$$
A^{\text {total-physical }}=A^{\text {ext }}+A^{v-\text { ind }}
$$

and define a response matrix $\Pi^{v}$ (sometimes called the Polarization) that gives the proportionality between the current and the total-physical vector potential via

$$
j=e \Pi^{v} A^{\text {total-physical }} .
$$

The superscript ${ }^{v}$ is included here to distinguish this polarization from one defined below when we consider the Chern-Simons problem, and also to remind us that we are looking at a response to the total field that includes the selfconsistent Coulomb $(v)$ interaction. In other words, $\Pi^{v}$ gives the density and current response to the total physical field including both the external field and the field internally induced by the Coulomb interaction. Indeed, when one attaches leads to the sample and measures a voltage, one is actually measuring this total field. Since the conductivity is defined as the response to the total field (being that that is what is measured with a voltmeter), $\Pi^{v}$ must be closely related to conductivity. This connection will be made more clear in the next section.

eThe use of the long phrase "total physical" may seem a bit cumbersome here, but it will help keep us from getting confused later. 
Using Eqs. 38, 44, 46, and 47 we can easily derive the matrix equation (dependences on $q$ an $\omega$ are implied)

$$
K^{-1}=\left[\Pi^{v}\right]^{-1}+V
$$

which can also be written as ${ }^{f}$

$$
K=\Pi^{v}-\Pi^{v} V \Pi^{v}+\Pi^{v} V \Pi^{v} V \Pi^{v}-\ldots
$$

In terms of diagrams, we say that $K$ is a chain sum of $\Pi^{v}$ connected by $V$ propagators, or equivalently, that $\Pi^{v}$ is the $V$-irreducible part of $K$. Sometimes it is said that $\Pi^{v}$ is the response $K$ with the Hartree part of the interaction "separated out". All of these are equivalent ways of saying that $K$ is the response to the externally applied field, whereas $\Pi^{v}$ is the response to the total field including external field and that induced internally by $v(\mathbf{q})$.

Note that with some amount of algebra one can derive from the matrix equation Eq. 48 the simpler statement

$$
K_{00}^{-1}=\left[\Pi_{00}^{v}\right]^{-1}+v
$$

which is what we would have naturally found if we had only kept track of the density $j_{0}$ and the scalar potential $A_{0}$ and not considered the full matrix response $K_{\mu \nu}$. It is worth noting that the relation derived here between $K$ and $\Pi^{v}$ is a very general relation that is always true for any system (by definition).

\subsection{Relation of Response to Resistivity and Conductivity}

As mentioned above, the response function $\Pi^{v}$ is the response to the total physical field including both the external field and the field internally induced by the Coulomb interaction. Since this total physical field is exactly the field that is measured experimentally by a voltmeter, we suspect that $\Pi^{v}$ can be related to the conductivity of the system. To make this connection apparent, we write the electric field in terms of the vector potential as $\mathbf{E}=\nabla A_{0}-\frac{d}{d t} \mathbf{A}$ or in Fourier space as (recall that we have chosen Coulomb gauge $\mathbf{A}=A_{1} \hat{\mathbf{y}}$ ) $E_{x}=-i q A_{0}$ and $E_{y}=-i \omega A_{1}$. We will also use the current conservation equation $q j_{x}=\omega j_{0}$ to convert between $j_{x}$ and $j_{0}$. It is now convenient to define a conversion matrix

$$
T=e\left[\begin{array}{cc}
\frac{i \sqrt{i \omega}}{q} & 0 \\
0 & \frac{1}{\sqrt{i \omega}}
\end{array}\right]
$$

\footnotetext{
${ }^{f}$ The - signs here are a function of our conventions for the charge of the electron and our use of $e$ in Eq. 38.
} 
This matrix will be used to apply the correct factors of $q$ and $\omega$ for converting between vector potential $A=\left(A_{0}, A_{y}\right)$ and electric field $\mathbf{E}=\left(E_{x}, E_{y}\right)$ and for converting between the vector $j=\left(j_{0}, j_{y}\right)$ and the vector $\mathbf{j}=\left(j_{x}, j_{y}\right)$. With this definition, we have

$$
\begin{aligned}
\mathbf{E} & =(-i \sqrt{-i \omega}) T^{-1} A \\
\mathbf{j} & =(-i \sqrt{-i \omega}) T j
\end{aligned}
$$

This conversion matrix will be used to switch freely between the two notations.

Using this conversion, we can rewrite Eq. 47 as

$$
\mathbf{j}=\sigma \mathbf{E}^{\text {total-physical }}
$$

where the matrix $\sigma$ is given by ${ }^{g}$

$$
\sigma=\rho^{-1}=T \Pi^{v} T .
$$

Since the matrix $\sigma$ relates the current to the total physical electric field (that measured by a voltmeter) this is what we call the conductivity matrix. It will be convenient at times to note that the longitudinal (00 or $x x$ ) part of this matrix equation implies

$$
\sigma_{x x}=\frac{\omega}{i \mathbf{q}^{2}} \Pi_{00}^{v} .
$$

\subsection{General Theme and Simple Example of RPA}

So far, we have not made any progress in calculating the response $K$; all we have done is to separate $K$ into pieces. Actually calculating $K$ or $\Pi^{v}$ is impossible for most nontrivial systems without making some sort of approximation. One of the simplest nontrivial approximations we can use to calculate a response is the RPA. The philosophy behind this approximation is to treat the interaction (such as $v$ ) by separating out the Hartree piece (such as in Eq. 48). Once this interaction piece is "taken care of", one approximates that which is left behind $\left(\Pi^{v}\right)$ as the response of noninteracting fermions. Another way of saying this is to say that at RPA level the electrons respond just as if they were noninteracting electrons - only they respond to both the external field and the self-consistent induced field also (reminiscent of our Chern-Simons RPA from section 3.1 above).

\footnotetext{
${ }^{g}$ In reference 14 a slightly different definition of the conductivity was used so as to obtain a result whose semiclassical approximation is well defined in the low frequency limit.
} 
The general rule for performing RPA is then the following. First we should write our Hamiltonian as

$$
H=H_{0}+H_{\text {int }},
$$

where $H_{0}$ is the Hamiltonian for noninteracting fermions. We then separate out $H_{\text {int }}$ at Hartree level to define a polarization $\tilde{\Pi}$

$$
K^{-1}=\tilde{\Pi}^{-1}+[\text { Interaction }] .
$$

For example, above, we have separated out the Coulomb term $v(q)$ and defined the polarization $\Pi^{v}$ in Eq. 48. However, when we have a system with more complicated interactions, we would want to separate out these pieces too. Once we have made this separation, approximating $\tilde{\Pi}$ as the response of noninteracting (mean field system) fermions (which we call $K^{0}$ ) yields the RPA response.

As a demonstration of this approach, we will show how to calculate $K$ for a system of electrons at $B=0$ (leaving the world of quantum Hall for the time being). Here, $v(q)$ is the only interaction. As described above, in the RPA, the polarization $\Pi^{v}$ is approximated as the response of noninteracting electrons, $K^{0}$ (discussed above in section 3.2). Using Eq. 50, we then obtain the RPA expression

$$
K_{00}=\frac{1}{\left[K_{00}^{0}\right]^{-1}+v(q)}
$$

This approach is a time honored method for understanding the $B=0$ electron gas.

\subsection{Chern-Simon RPA (Again)}

We now return to the world of high magnetic fields and Chern-Simons fermions. We will re-derive the Chern-Simons RPA discussed above in section 3.1 in terms of the response function $K$. The theme of separating out the Hartree part of the interaction will pervade the rest of this discussion - only now we will worry about the Chern-Simons interaction as well as the Coulomb interaction.

In the Chern-Simons approach, when we make the mean field approximation, we fix the density and currents in the system to be everywhere constant such that the Chern-Simons magnetic field is a fixed constant and the Coulomb interaction is a constant. When we go beyond mean field, we look at the effect of the density or currents fluctuations coupling to the interactions. Analogous to the Hartree separation of the Coulomb interaction that we discussed above, we will define new response functions in this section that separate out the Chern-Simons interaction. 
As we discussed above (See Eqs 25 and 26), in the Chern-Simons theory an excess density $j_{0}$ carries an excess Chern-Simons flux of $\tilde{\phi}=2 m$ flux quanta per particle resulting in an excess Chern-Simons magnetic field $\delta b=\tilde{\phi} \phi_{0} j_{0}$. Similarly a composite fermions' current $\mathbf{j}$ carries a current of flux tubes, inducing a Chern-Simons electric field $\mathbf{e}=-2 \pi \tilde{\phi} \frac{e}{c} \hat{\mathbf{z}} \times \mathbf{j}(\mathbf{q})$.

It is convenient now to write these results in terms of the vector potential, (Again, we have the convention that the wavevector $\mathbf{q} \| \hat{\mathbf{x}}$; we also will typically drop the matrix indices and the explicit $q$ and $\omega$ dependences)

$$
e A^{C S-\text { ind }}=-C j
$$

where the superscript ${ }^{C S}$ stands for Chern-Simons and the interaction matrix is given by ${ }^{h}$ (See Eqns. 26 and 52)

$$
C=T \rho_{\mathrm{CS}} T=\frac{2 \pi \tilde{\phi} \hbar}{e}\left[\begin{array}{cc}
0 & \frac{i}{q} \\
\frac{-i}{q} & 0
\end{array}\right] .
$$

Recalling that a vector potential $A_{v}^{\text {ind }}=V j$ is induced by the Coulomb interaction, we have

$$
A^{\text {total }}=A^{\text {ext }}+A^{v-\text { ind }}+A^{\mathrm{CS}-\text { ind }} .
$$

We emphasize again that the induced Chern-Simons vector potential is not a physical field measured by a voltmeter since it is only seen by other transformed fermions within the two dimensional electron gas. However, the transformed fermions do respond to this Chern-Simons piece.

We now define a response function $\Pi$ that gives the current and density response to this total field ${ }^{i}$.

$$
j=e \Pi A^{\text {total }} .
$$

We can now combine Eqns. 63, 38, 44, and 60 to obtain the relation

$$
K^{-1}=\Pi^{-1}+V+C
$$

Thus the definition of $\Pi$ separates out the Hartree part of both the Coulomb and the Chern-Simons interactions. In terms of a diagrammatic expansion, $\Pi$ is defined as the contribution from all Feynman diagrams for $K$ that are

\footnotetext{
${ }^{h}$ Note that $\mathrm{HLR}^{14}$ call this $C^{-1}$.

${ }^{i}$ Our matrix $\Pi$ is written as $\tilde{K}$ in references 14 and 16 . However, our notation for $\Pi$ agrees with that used in references $17,18,43$, and 44 . The other common notation is to define $\Pi=T \sigma T$ which is what we call $\Pi^{v}$
} 
irreducible with respect to both the Coulomb interaction $V$ and the ChernSimons interaction $C$. Here $\Pi$ should be though of as the composite fermion response, analogous to $\sigma_{\mathrm{CF}}$ discussed above in section 3.1. Indeed, using Eqs. $64,61,55,48$, and 30 we have

$$
\rho_{\mathrm{CF}}=[T \Pi T]^{-1}
$$

We note that Eq. 64 has the general form of separation as defined in Eq. 58. Thus, in terms of the response function $K$, the RPA is analogously obtained by setting $\Pi$ to be $K^{0}$ the response for the noninteracting mean field system, such that we have the RPA prescription

$$
K^{-1}=\left[K^{0}\right]^{-1}+V+C
$$

Results of RPA calculations for the Jain fractions $\nu=\frac{p}{2 m p+1}$ such that $\Delta B \neq 0$ are discussed at length in Refs. 13 and 16.

\subsection{Results of Chern-Simons RPA at $\nu=\frac{1}{2 m}$}

At this point, we will turn our focus to the even denominator states $\nu=\frac{1}{2 m}$. The mean field system is then in zero magnetic field (such that $K^{0}$ is diagonal). We can now plug $K^{0}$ into Eq. 66 to obtain

$$
K_{00}=\frac{1}{\left(\frac{2 \pi \tilde{\phi}}{q}\right)^{2} K_{11}^{0}+\left[K_{00}^{0}\right]^{-1}+v(q)}
$$

We note that this looks exactly like the RPA response $K_{00}$ for a normal Fermi gas in zero magnetic field (Eq. 59) except for the presence of the $K_{11}^{0}$ term in the denominator. The full forms of $K^{0}$ for fermions in zero magnetic field are given in appendix A.

We will now examine the results of the Chern-Simons RPA at low frequency $\omega \ll q v_{\mathrm{F}}$. Plugging in the low frequency form of $K^{0}$ (given by Eqns A.3 and A.4) into Eq. 67 yields (here we set the mass of the fermion to be $m_{\mathrm{b}}$ as it should be in the RPA approximation),

$$
K_{00}(q, \omega)=\frac{1}{\frac{2 \pi}{m_{\mathrm{b}}}\left[1+\frac{\tilde{\phi}^{2}}{12}\right]+v(q)+i\left(\frac{2 \pi \tilde{\phi}}{q}\right)^{2}\left(\frac{2 \hbar n_{\mathrm{e}}}{m_{\mathrm{b}}}\right) \frac{\omega}{q v_{\mathrm{F}}}}
$$

At zero frequency, this expression looks very similar to the RPA response of fermions in zero magnetic field ${ }^{j}$. However at finite frequency this response has

${ }^{j}$ At zero field, using Eq. A.3 in Eq. 59, we obtain

$$
K_{00}(q, \omega)=\frac{1}{\frac{2 \pi}{m_{\mathrm{b}}}\left[1+\frac{i \omega}{q v_{\mathrm{F}}}\right]+v(q)}
$$


a pole at imaginary frequency

$$
\omega \sim i q^{3} v(q)
$$

which does not occur for the usual Fermi gas at zero field. This so-called "overdamped mode" is diffusive (i.e., $\sim q^{2}$ ) for Coulomb interactions and subdiffusive $\left(\sim q^{3}\right)$ for short range interactions, and represents a slow (or extremely slow in the case of short range interactions) relaxation of density fluctuations. One might expect this slow relaxation in large magnetic field since an electron being pushed away from a region of high density by the Coulomb interaction ends up moving perpendicular to the force, thus not relaxing the density at all. It is also natural that the density relaxation should be slower in the case of short range interaction where the interaction cannot move density at greater distances. The existence of this overdamped mode is what causes the infra-red divergences in section 6.3 below.

For higher frequencies, we look back to the noninteracting case for insight. For noninteracting fermions, at finite wavevector $q$, there is a continuum of low energy excitations that one can make by exciting a single fermion out of the Fermi sea by wavevector $q$. This can create excitations up to a maximum energy $\omega_{\text {cutoff }}=\frac{1}{2 m}\left[\left(q+k_{\mathrm{F}}\right)^{2}-k_{\mathrm{F}}^{2}\right] \approx q v_{\mathrm{F}}$. Similarly for the composite fermion Fermi liquid, we have the same cutoff to the low energy band (this is obvious, since in Eq. 66 the noninteracting $K^{0}$ must have an imaginary part in order for $K$ to have an imaginary part). However, unlike the noninteracting case, there is also a cyclotron mode at high energy, and the weight of the low energy continuum is bunched down at very low energy $\omega \sim q^{3} v(q)$.

In Fig. 1 we show the excitation spectrum of the $\nu=\frac{1}{2}$ state calculated in RPA. In (a) the edge of the continuum is shown solid, the cyclotron mode (See Eq. 73) is shown dashed, and the maximum of the weight in the continuum is shown dotted. In (b) the structure factor is shown explicitly as a function of frequency for several wavevectors. Note that the RPA is only expected to be valid at small $q$, but we have displayed it here to larger $q$ for clarity.

\subsection{Sum Rules}

It is instructive to stop at this point and examine some of the properties we expect our response functions to have - particularly at high frequency. To begin with, we recall that Kohn's theorem ${ }^{27}$ (a result of Galilean invariance) requires that the $q \rightarrow 0$ behavior of our system be determined by the band mass

which is quite similar to the HLR-RPA form above at $\omega=0$ (the only difference being that the term $\tilde{\phi}^{2} / 12$ is missing). We note that the imaginary frequency pole here is at $\omega \sim i v(q) q$ which yields very different low energy physics from that of the Chern-Simons Fermi liquid. 

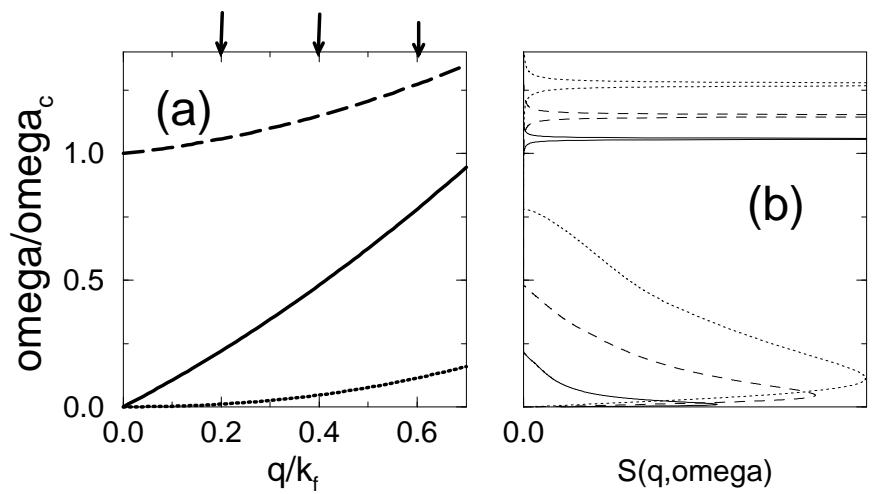

Figure 1: Excitation Spectrum of $\nu=\frac{1}{2}$ in RPA

In (a) the solid line is the edge of the low energy continuum of quasiparticles, the dotted line is the location of the peak in the weight (the maximum of the structure factor) of the low energy continuum $\left(\omega \sim q^{3} v(q)\right)$, and the dashed line is the cyclotron mode. In (b) the structure factor $S(q, \omega)$ is shown explicitly for three different wavevectors $q / k_{\mathrm{F}}=.2$ (solid), .4. (dashed), and .6 (dotted). The amplitude of $S$ is the horizontal direction and the frequency axis is the same scale as for (a). Note that the peak is at very low frequency. A small broadening is used so the sharp cyclotron mode can be seen. Note that the integrated weight of the structure factor is proportional to $q^{2}$ in accordance with the $f$-sum rule. Here we have used $E_{\mathrm{c}} /\left(\hbar \omega_{\mathrm{c}}\right)=5$ which is large if we use the bare mass, but reasonable if we use a renormalized mass in $\omega_{\mathrm{c}}$. 
$m_{\mathrm{b}}$ rather than any renormalized mass. One can imagine all of the electrons in the system oscillating in unison so that electron-electron interactions have no effect. More formally, one finds that the motion of the center of mass degree of freedom of the system decouples from any interactions between the particles so that in the long wavelength limit, interactions do not effect the response of the system.

Similarly the $f$-sum rule ${ }^{29-31}$ simply says that the behavior of our system in the $\omega \rightarrow \infty$ limit is also determined by the band mass $m_{\mathrm{b}}$. This is easily imagined since at high frequency one can think of the electrons oscillating very quickly with very small magnitude so that these oscillations do not appreciably change the positions of the electrons or couple to the electron-electron interaction. We note that the $f$-sum rule is stronger than Kohn's theorem in the sense that it hold also in a disordered system.

We can summarize both of these rules by stating that in the long wavelength or high frequency limit, the resistivity of any system should look like

$$
\rho \sim \frac{m_{\mathrm{b}}}{e^{2} n_{\mathrm{e}}}\left[\begin{array}{cc}
-i \omega & \omega_{\mathrm{c}} \\
-\omega_{\mathrm{c}} & -i \omega
\end{array}\right]+\mathcal{O}\left(q^{2} / \omega\right)
$$

If the resistivity of our system indeed has this high frequency, long wavelength limit, then Kohn's theorem and the $f$-sum rules are satisfied ${ }^{k}$.

We now check that the RPA approximation satisfies this condition. The mean field resistivity of the system is just the resistivity of a system of free fermions of mass $m_{\mathrm{b}}$ in a field $\Delta B$, and should thus (by Kohn's theorem and the $f$-sum rule for free fermions) have the form

$$
\rho_{\text {mean-field }} \sim \frac{m_{\mathrm{b}}}{e^{2} n_{\mathrm{e}}}\left[\begin{array}{cc}
-i \omega & \Delta \omega_{\mathrm{c}} \\
-\Delta \omega_{\mathrm{c}} & -i \omega
\end{array}\right]+\mathcal{O}\left(q^{2} / \omega\right)
$$

where $\Delta \omega_{\mathrm{c}}=e \Delta B /\left(m_{\mathrm{b}} c\right)$ is the cyclotron frequency associated with the effective magnetic field and the band mass. Defining the mean field resistivity to be the composite fermion resistivity $\rho_{\mathrm{CF}}$, using Eq. 30 to calculate the electron resistivity $\rho$, and using the fact that (Eq. 17) $\Delta B=B-\phi_{0} \tilde{\phi} n_{\mathrm{e}}$, we can easily show that the RPA resistivity also satisfies Kohn's theorem and the

\footnotetext{
${ }^{k}$ Often the $f$-sum rule is stated in terms of the conductivity $\sigma$ or the electromagnetic response $K$. By using a Kramers-Krönig relation, this high frequency condition can be written as an integral over frequency such as

$$
\int_{-\infty}^{\infty} d \omega \operatorname{Re} \sigma_{x x}(q, \omega)=\pi n_{\mathrm{e}} / m_{\mathrm{b}} .
$$

It is tempting to think that the name " $f$-sum" comes from this sum over frequencies, but in truth the name is (once again) more of a historical accident
} 
$f$-sum rule. Indeed, what we find is that the RPA resistivity satisfies these sum rules if and only if the composite fermion resistivity $\rho_{\mathrm{CF}}$ satisfies these sum rules with respect to the effective magnetic field (i.e., if the composite fermion resistivity has same large frequency small wavevector form as the mean field result of Eq. 72).

It is interesting to note that by using the long wavelength, high frequency form of the resistivity fixed by the $f$-sum rule (Eq. 70), we can calculate the response function $K_{00}$ by using Eqs. 70, 55, and 50. Once we have this form we can find the excitation spectrum by looking for poles of the response matrix $K_{00}$, which are then given by

$$
\omega^{2}=\omega_{\mathrm{c}}^{2}+\frac{q^{2} v(q) n_{\mathrm{e}}}{m_{\mathrm{b}}}
$$

which is a completely general result ${ }^{l}$. Note that in zero magnetic field $\omega_{\mathrm{c}} \rightarrow 0$, this Kohn mode goes continuously into the plasma mode.

\subsection{Energy Scales, Effective Mass, and a Problem with RPA}

As mentioned above in section 2.3.2, at mean field level, the excitation gap for the fractional Hall state is given by $\Delta \omega_{\mathrm{c}}=\frac{\hbar e \Delta B}{m_{\mathrm{b}} c}$ which is not correct. At RPA level, this remains a problem. To explain in more detail why this is a such a serious problem, we should carefully examine the energy scales of the problem.

The natural energy scale for the interaction strength ${ }^{m}$ is $v\left(l_{B}\right)$ with $v$ the interelectron interaction potential and $l_{B}$ the magnetic length. For the case of the Coulomb interaction, this energy scale is thus (the subscript ${ }_{\mathrm{c}}$ is for Coulomb)

$$
E_{c}=e^{2} /\left(\epsilon l_{B}\right)
$$

which is proportional to $\sqrt{B}$. On the other hand, the cyclotron energy (the spacing between Landau levels) is $\hbar \omega_{\mathrm{c}} \sim B / m_{\mathrm{b}}$. Thus, a natural simplification to make is to assume the limit of large magnetic field (or sometimes $\left.m_{\mathrm{b}} \rightarrow 0\right)$ such that the cyclotron energy is much greater than the interaction $\operatorname{energy}^{n}$. In this case, we can assume that the inter-Landau-level excitations

\footnotetext{
${ }^{l}$ Other corrections occur at order $q^{2}$.

${ }^{m}$ Note that $l_{B}$ is considered to be the natural length scale of the problem. One might also consider defining $1 / \sqrt{n_{\mathrm{e}}}$ to be the natural length scale. This differs from $l_{B}$ only by a factor of $(2 \pi / \nu)^{1 / 2}$.

${ }^{n}$ In practice, for typical GaAs band mass, the cyclotron energy becomes greater than the interaction energy at roughly 8 Tesla. However, it is also thought that the main effect of the higher Landau levels is to screen the Coulomb interaction (by allowing virtual inter-Landaulevel transitions) so that even when $\hbar \omega_{c}$ is not much greater than the interaction energy, we can still consider only the physics of the lowest Landau level.
} 
are energetically forbidden so that we need only consider states within a single Landau level (within the lowest Landau level for the case of $\nu<1$ ). Once the problem is reduced to a single Landau level, the only energy scale remaining in the problem is the Coulomb energy ${ }^{o}$. It is worth noting that all of the exact diagonalizations calculations, as well as all trial wavefunctions, are studied in this limit ${ }^{6,28,26,23}$. In this limit, the excitation gap for the fractional quantum Hall state must clearly be on order of the interaction energy $\left(e^{2} / \epsilon l_{B}\right)$. This is quite natural being that the correlation effect, and the fractional Hall state itself, is entirely due to the interelectron interactions.

As discussed above, however, in the Chern-Simons picture at the mean field level, the fractional quantum Hall gap is given by $\Delta \omega_{\mathrm{c}}=\hbar e \Delta B /\left(m_{\mathrm{b}} c\right)$ which is completely independent of the interaction strength, and is thus clearly incorrect. At RPA level, the low energy excitations remains on the scale of $\Delta \omega_{\mathrm{c}}$. It should be immediately clear that we can not obtain a gap on the scale of the interaction strength at RPA level since we were able to define the essence of the RPA (in section 3.1) without even mentioning the Coulomb interaction! It is also clear that this problem is serious since for typical experimental parameters ${ }^{24} \Delta \omega_{\mathrm{c}}$ can be 4 to 15 times the measured energy gap! In order to fix this problem we will need an approximation beyond RPA.

One naive approach we might immediately try is to just assume that the composite fermion mass becomes renormalized from the bare band mass $m_{\mathrm{b}}$ to some renormalized mass $m^{*}$. This is not such a strange thing to assume since we know that this is a highly interacting system and interactions often renormalize quantities such as the mass of particles. It thus seems reasonable to assume the mass is somehow renormalized and to treat $m^{*}$ as an experimentally measured phenomenological parameter without worrying too much about exactly how it gets renormalized. Indeed, such an assumption has been made in calculations by many different groups. The problem with such an approach, however, is that the resulting calculations violate the $f$-sum rule and Kohn's theorem. To see this, we imagine replacing $m_{\mathrm{b}}$ with $m^{*}$ everywhere it occurs. We then calculate the composite fermion resistivity $\rho_{\mathrm{CF}}^{\text {naive }}$ and find that it is now given by (compare to Eq. 72)

$$
\rho_{\mathrm{CF}}^{\text {naive }} \sim \frac{m_{\mathrm{b}}}{e^{2} n_{\mathrm{e}}}\left[\begin{array}{cc}
-i \omega & \Delta \omega_{\mathrm{c}}^{*} \\
-\Delta \omega_{\mathrm{c}}^{*} & -i \omega
\end{array}\right]+\mathcal{O}\left(q^{2} / \omega\right)
$$

${ }^{o}$ It should be noted, however, that such projection to the lowest Landau level is not without risk. See section 5 below as well as Ref. 65 for some problems that occur when one tries to work in only a single Landau level. 
where now we have the effective cyclotron frequency

$$
\Delta \omega_{\mathrm{c}}^{*}=\frac{e \Delta B}{m^{*} c}
$$

which now differs from the mean field result. As discussed above, the electron system will satisfy Kohn's theorem and the $f$-sum rule, if and only if the composite fermion resistivity $\rho_{\mathrm{CF}}$ has the same limiting form as the mean field result Eq. 72. Since this is not the case, we see that this naive approach must violate these sum rules. In order to satisfy these sum rules and obtain the correct energy scales for excitations, we will have to consider a more complicated, and somewhat phenomenological, approximation which we will discuss in the next section.

\section{Landau Fermi Liquid Theory and MRPA}

In section 3, we found that the RPA gave many results correctly, but it still did not give us the correct energy scale for low energy excitations. Furthermore, as we saw in section 3.9 above, straightforward renormalization of the fermion mass $m_{\mathrm{b}}$ to some renormalized value $m^{*}$ resulted in violation of Kohn's theorem and the $f$-sum rule.

Fundamentally, the reason we are having trouble with RPA is that the Chern-Simons system is highly interacting. In particular, the Chern-Simons interaction (the fact that one fermion sees, and responds to, the flux attached to all of the other fermions) is quite strong. In perturbative approaches such as RPA ${ }^{a}$, we attempt to identify some small parameter (much less than one) in which to expand. Here, there is no small parameter. We will see below in section 6.3 that the closest thing we have to a small parameter is $\tilde{\phi}$, the number of flux quanta attached to each fermion, which is at least 2 and is clearly not small.

Fortunately, many highly interacting fermion systems have been successfully studied in the past. Although the Chern-Simons interaction between composite fermions is different from the interactions encountered in well studied fermionic systems such as Helium-3 or electrons in metals, it is reasonable to expect that some version of Fermi liquid theory ${ }^{29,30}$ will be applicable. In this section, we will discuss the phenomenological Fermi liquid theory and how it can cure some of the illnesses of the Chern-Simons RPA.

The knowledgeable reader might object that framing the Chern-Simons fermion theory in the language of traditional simple Fermi liquid theory com-

\footnotetext{
${ }^{a} \mathrm{RPA}$ can be thought of as the lowest order perturbative correction to mean field made self-consistent
} 
pletely misses the most interesting pieces of physics. In particular, one might object that infra-red divergences actually make the system non-Fermi liquid (or "marginally" Fermi liquid) in several ways, and therefore should not be described within the Fermi liquid framework. Admittedly, these infra-red divergences do lead to a very non-traditional system and we will discuss these issues in depth in section 6.3 below. However, for now, we will be concerned with seeing how well we can describe the system within this traditional framework. For the reader who is worried about our blithe neglect of these important issues, we point out that we can justify this neglect and sweep these infra-red issue under the rug by considering the case of long range interactions. If we assume that the inter-electron interaction $v(r)$ is longer range than Coulomb (for example, a power law form $v(r) \sim r^{-\eta}$ with $\eta<1$ ) then the long wavelength density fluctuations become suppressed and the system no longer has these infra-red problems (We will discuss this issue more in section 6.3 below). Although this hypothetical long range interaction clearly does not correspond to any real electron system ${ }^{b}$ it is nonetheless useful to think about this simple case. Once we have fully analyzed this more simple case, we will return to the physical situation and worry about these additional complications in sections 6.3 and 6.4 below.

The essence of Landau's Fermi liquid theory is that the long wavelength low frequency response of a system of fermions in a Fermi liquid phase can be deduced from an effective low energy description of weakly interacting quasiparticle excitations near a Fermi surface. As with free fermions, it is assumed that the ground state is a filled Fermi sea, and quasiparticles excited from below the Fermi level to above constitute the elementary excitations. These quasiparticles are allowed to have a renormalized effective mass $m^{*}$, and some local interactions described by a function $f$ that we will define more carefully below. In general, the properties of these quasiparticles can be quite different from the properties of the bare particles in the system. For example, in the Fermi liquid phase of Helium-3, the effective mass of the quasiparticle is over 3 times as large as that of the bare Helium-3 fermion. A similar thing occurs for the Chern-Simons fermion system. We we will find that, within a Fermi liquid theory description, we can have a quasiparticle mass that is very different from the bare mass (being set by the interaction energy) and we can still satisfy the necessary sum rules (Kohn's theorem and the $f$-sum rule). Furthermore, we will see in section 5 that an additional complication associated with what we call magnetization currents can also be solved within this Fermi liquid format by allowing the Fermi liquid quasiparticle to become

\footnotetext{
${ }^{b}$ See however, Ref. 32 in which it is shown that a Josephson junction array is analogous to a quantum Hall system with long range interactions.
} 
magnetized. Perhaps most importantly, this Fermi liquid description will allow us to calculate response functions that can then be compared to experiment.

Section 4.1 is an extended review of basic Fermi liquid theory. Those who are quite familiar with these topics are encouraged to skip over this section, reading only that which is necessary to become familiar with the notation. In section 4.2 we will discuss the Landau-Silin extension of Fermi liquid theory for Fermi liquids with long range interactions. This will, of course, be directly applied to the case at hand of our composite fermions interacting via a long ranged Coulomb interaction as well as a Chern-Simons interaction. Finally, in section 4.3 we discuss the MRPA approximation for calculation of response functions for the Chern-Simons Fermi liquid. The main physics of MRPA is that by including a Landau $F_{1}$ coefficient we can renormalize the mass scale of the RPA calculation while preserving Kohn's theorem and the $f$-sum rule.

\subsection{Conventional Fermi Liquid Theory}

In the Landau Fermi liquid theory, the state of the system is described by the function $\delta n(\mathbf{p}, \mathbf{r})$, characterizing the difference of the density of fermions at the phase space point $\mathbf{p}, \mathbf{r}$ between an excited state and the ground state ${ }^{c}$. The function $\delta n(\mathbf{p}, \mathbf{r})$ fully describes the density and current density anywhere in the system. Thus, to calculate the response of the system to a force, one need only find the behavior of this function $\delta n(\mathbf{p}, \mathbf{r})$. Note that in the case of a charged Fermi liquid, we should consider $\mathbf{p}$ to be the canonical ${ }^{30,29}$ momentum $\hbar \mathbf{k}-\frac{e}{c} \mathbf{A}$ with $\mathbf{A}$ the vector potential.

In this section we will review the basics of Landau's theory for systems with short range interactions and we will worry about the complications involved in adding the long range Coulomb and Chern-Simons interactions below in section 4.2 .

\subsubsection{Fermi Liquid Basics}

Fermi liquid theory states that a weak long wavelength low frequency driving force affects only the occupation of states near the Fermi momentum. The energy cost associated with a deviation $\delta n(\mathbf{p}, \mathbf{r})$ of the phase space distribution function from its ground state value is given by a phenomenological energy density functional

$$
\mathcal{E}[\delta n(\mathbf{p}, \mathbf{r})]=\int \frac{d \mathbf{p}}{(2 \pi \hbar)^{2}} \epsilon_{0}(\mathbf{p}) \delta n(\mathbf{p}, \mathbf{r})
$$

\footnotetext{
${ }^{c}$ Needless to say, such a phase space density is only defined semiclassically since $\mathbf{p}$ and $\mathbf{r}$ don't commute as operators.
} 


$$
+\frac{1}{2} \int \frac{d \mathbf{p}}{(2 \pi \hbar)^{2}} \int \frac{d \mathbf{p}^{\prime}}{(2 \pi \hbar)^{2}} f\left(\mathbf{p}, \mathbf{p}^{\prime}\right) \delta n(\mathbf{p}, \mathbf{r}) \delta n\left(\mathbf{p}^{\prime}, \mathbf{r}\right) .
$$

Here $\epsilon_{0}=\mathbf{p}^{2} / 2 m^{*}$. Thus, this energy functional depends on only two parameters - the quasiparticle effective mass, $m^{*}$ which determines the energy of exciting a single quasiparticle above the Fermi surface, and the Landau interaction function $f\left(\mathbf{p}, \mathbf{p}^{\prime}\right)$ that gives the added quasiparticle-quasiparticle interaction energy associated with having more than one quasiparticle excited. Note that this interaction functional can only describe short range interactions (those local in $\mathbf{r}$ ). The two parameters $m^{*}$ and $f$ in the energy functional (Eq. 77) are formally defined in terms of one and two particle Green's functions ${ }^{30}$. These definitions are not essential for this part of our discussion, though we will return to this issue in section 6.3 below.

The quasiparticle energy $\tilde{\epsilon}$ is obtained by differentiating Eq. 77 with respect to $\delta n(\mathbf{p}, \mathbf{r})$, yielding,

$$
\tilde{\epsilon}(\mathbf{p}, \mathbf{r})=\epsilon_{0}(\mathbf{p}, \mathbf{r})+\int \frac{d \mathbf{p}^{\prime}}{(2 \pi \hbar)^{2}} f\left(\mathbf{p}, \mathbf{p}^{\prime}\right) \delta n\left(\mathbf{p}^{\prime}, \mathbf{r}\right) .
$$

The quasiparticle velocity is then naturally given as $\mathbf{u}(\mathbf{p})=\nabla_{\mathbf{p}} \tilde{\epsilon}$, such that we can write the particle density and current in terms of the phase space density

$$
\begin{aligned}
n(\mathbf{r}) & =\int \frac{d \mathbf{p}}{(2 \pi \hbar)^{2}} \delta n(\mathbf{p}, \mathbf{r}) \\
\mathbf{j}(\mathbf{r}) & =\int \frac{d \mathbf{p}}{(2 \pi \hbar)^{2}} \delta[\mathbf{u}(\mathbf{p}) n(\mathbf{p}, \mathbf{r})] .
\end{aligned}
$$

To calculate a response, we should use a Boltzmann transport equation derived from the energy functional. Most generally, a Boltzmann equation is difficult to solve. However, we will be able to make use of the fact that in Fermi liquid theory, the relevant ${ }^{d}$ low energy excitations are smooth fluctuations of the shape of the Fermi surface. To this end, we consider fluctuations of the shape of the Fermi surface that we describe as ${ }^{e}$

$$
n(\mathbf{p}, \mathbf{r})=n_{0}\left[p-p_{\mathrm{F}}-\nu(\theta, \mathbf{r})\right]
$$

where $\theta$ is the direction of $\mathbf{p}$ on the Fermi surface, $p_{\mathrm{F}}$ is the Fermi momentum, and $n_{0}$ is the equilibrium phase space density (taken to be a step function at

\footnotetext{
${ }^{d}$ Here we use the word "relevant" in the renormalization group sense as well as in the conventional sense of the word. A good review of how renormalization group can be applied to Fermi systems to obtain Fermi liquid theory is given in Ref. 33.

${ }^{e}$ Note that definition of $\nu$ used here agrees with that in Refs. 43, 30, and 29 , but differs from the function $f$ used in references 35 and 16 by a factor of $v_{\mathrm{F}}^{*}=p_{\mathrm{F}} / m^{*}$.
} 
zero temperature, and an appropriate Fermi occupation at finite temperature). Thus, the function $\nu(\theta, \mathbf{r})$ describes the shape of the Fermi surface ${ }^{f}$. We will use the convention that $\theta=0$ points in the $\hat{\mathbf{x}}$ direction.

Since we are only concerned with small excitations of the Fermi surface, we assume that the interaction function $f\left(\mathbf{p}, \mathbf{p}^{\prime}\right)$ is not a function of the magnitude of the vectors $\mathbf{p}$ and $\mathbf{p}^{\prime}$ (since all magnitudes are approximately $p_{\mathrm{F}}$ ) and is only a function of their directions ${ }^{g}$. Furthermore, by rotational invariance, the function $f$ should only depend on only the angle between $\mathbf{p}$ and $\mathbf{p}^{\prime}$ so that in two dimensions we can write $f\left(\theta-\theta^{\prime}\right)$ where $\theta$ is the angle of $\mathbf{p}$ and $\theta^{\prime}$ is the angle of $\mathbf{p}^{\prime}$.

\subsubsection{Fourier Space and Restrictions on Fermi Liquid Coefficients}

It is often more convenient to work with Fourier transformed quantities. We now define

$$
\begin{aligned}
f_{l} & =\frac{1}{2 \pi} \int_{0}^{2 \pi} d \theta f(\theta) e^{i l \theta} \\
\nu_{l}(\mathbf{r}) & =\frac{1}{2 \pi} \int_{0}^{2 \pi} d \theta \nu(\theta, \mathbf{r}) e^{i l \theta} .
\end{aligned}
$$

Note that due to the symmetry of the interaction function $f(\theta)=f(2 \pi-\theta)$ we expect that $f_{l}=f_{-l}$. Note that a very common notation is to define the dimensionless quantity ${ }^{h}$

$$
F_{l}=\frac{m^{*} f_{l}}{2 \pi \hbar^{2}}
$$

Clearly, the zeroth Fourier mode corresponds to a uniform compression of the Fermi surface, which corresponds to the density change (See Eq. 79)

$$
\delta n(\mathbf{r})=\frac{p_{\mathrm{F}} \nu_{0}(\mathbf{r})}{2 \pi \hbar^{2}}+\mathcal{O}\left(\nu_{0}^{2}\right) .
$$

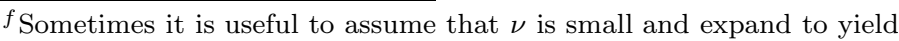

$$
\delta n(\mathbf{p}, \mathbf{r})=\nu(\theta, \mathbf{r}) \delta\left(|\mathbf{p}|-p_{\mathrm{F}}\right) .
$$

However, this must be used with caution since we may sometimes want the second order contributions.

${ }^{g}$ In the Chern-Simons case (with Coulomb or short range interactions), it is not so clear that $f\left(\mathbf{p}, \mathbf{p}^{\prime}\right)$ should depend only on the angle since the behavior of quasiparticles is singular as they approach the Fermi surface. This complication is discussed further in sections 6.3 and 6.4 below, although our understanding of this is far from complete. One hopes that in a fully renormalized theory (nonperturbatively) these singularities do not prevent us from writing a Boltzmann transport equation. We note that Kim et $\mathrm{al}^{44}$ recently showed that a form of quantum Boltzmann equation can be derived independent of these singularities.

${ }^{h}$ In references 16,36 , and $35, F_{l}$ is called $A_{l}$. 
The first Fourier mode, on the other hand, corresponds to a uniform boost of the Fermi surface resulting in the current density ${ }^{i}$

$$
\begin{aligned}
& j_{x}(\mathbf{r})=\frac{n_{\mathrm{e}}}{m_{\mathrm{b}}}\left(\nu_{1}(\mathbf{r})+\nu_{-1}(\mathbf{r})\right)+\mathcal{O}\left(\nu^{2}\right) \\
& j_{y}(\mathbf{r})=\frac{n_{\mathrm{e}}}{i m_{\mathrm{b}}}\left(\nu_{1}(\mathbf{r})-\nu_{-1}(\mathbf{r})\right)+\mathcal{O}\left(\nu^{2}\right) .
\end{aligned}
$$

Note that here we use the bare mass of the fermion since we are boosting the entire Fermi sea. Higher Fourier modes correspond to more complex deformations of the Fermi surface that carry no net current and no net charge.

In terms of these Fourier transformed quantities, the energy-density functional (Eq. 77) can be written simply as

$$
\mathcal{E}\left[\nu_{l}(\mathbf{r})\right]=\epsilon_{0}\left(p_{\mathrm{F}}\right) \delta n(\mathbf{r})+\sum_{l} \frac{n_{\mathrm{e}}}{m^{*}}\left(1+F_{l}\right)\left|\nu_{l}(\mathbf{r})\right|^{2} .
$$

This expression assigns to each mode of deformation of the Fermi surface a specific energy density which is made up of a noninteracting part (the " 1 " in the parenthesis) and an interaction contribution (the $F_{l}$ piece). It is worth noting that the $l^{\text {th }}$ Fourier deformation mode couples only to the interaction coefficient $F_{l}$. Thus, $F_{0}$ (or $f_{0}$ ) describes the interaction energy associated with the compression mode $l=0$ of the Fermi surface, whereas $F_{1}$ (or $f_{1}$ ) describes the interaction energy associated with the boost mode $(l=1)$. As such, we expect that $f_{0}$ should be related to the compressibility of the system, whereas $f_{1}$ should be related to the bare mass of the system (which should tell us the energy of boosting the entire mass of the system). Indeed, these expectations are true as we will now see.

By noting that $\mu=d \mathcal{E} / d(\delta n(\mathbf{r}))$, explicitly differentiating Eq. 88, and making use of Eq. 85 to relate $\nu_{0}$ to $\delta n(\mathbf{r})$, we obtain the compressibility equation $^{j}$

$$
\frac{d \mu}{d n}=\frac{2 \pi \hbar^{2}}{m^{*}}+f_{0}
$$

${ }^{i}$ To see this result more clearly, consider for example boosting the entire Fermi sea by a small vector $\mathbf{q}$, corresponding to a current density $\mathbf{j}=n_{\mathrm{e}} \mathbf{q} / m_{\mathrm{b}}$. A point $\mathbf{p}$ (at angle $\theta$ ) on the Fermi surface, then gets boosted to a point $\mathbf{p}+\mathbf{q}$ whose magnitude is $p_{\mathrm{F}}+\mathbf{p} \cdot \mathbf{q}+\mathcal{O}\left(q^{2}\right)$ thus giving $\nu(\theta)=\cos \left(\theta-\theta_{q}\right)$. Converting to Fourier space then yields $\nu_{1}+\nu_{-1}=q_{x}$ and $\nu_{1}-\nu_{-1}=i q_{y}$. One could have alternately inserted $\nu(\theta)$ into Eq. 80. However, in order to get the same result, we would then need Eq. 91 below.

${ }^{j}$ In the case of the Chern-Simons Fermi liquid, the compressibility derivative $\frac{d \mu}{d n}$ is taken at fixed $\Delta B$. This point is somewhat subtle, and is discussed in detail in sections 5.4.1 and 6.4 below. 
Similarly, boosting the entire system a momentum q, by Galilean invariance, should cost an energy density of $n_{\mathrm{e}} q^{2} /\left(2 m_{\mathrm{b}}\right)$. For convenience we choose $\mathbf{q}$ to be in the $\hat{\mathbf{x}}$ direction such that have $\nu_{1}=\nu_{-1}=\frac{1}{2} q$. We can now calculate the energy density of this boost in a different way by using Eq. 88 to obtain $\frac{2 n_{\mathrm{e}}}{m^{*}}\left(1+F_{1}\right)\left(\frac{q}{2}\right)^{2}$. Equating these two equivalent expression for the energy then yields

$$
\frac{m^{*}}{m_{\mathrm{b}}}=1+F_{1}
$$

or equivalently

$$
\frac{1}{m_{\mathrm{b}}}=\frac{1}{m^{*}}+\frac{f_{1}}{2 \pi \hbar^{2}}
$$

We note in passing that one can derive an additional sum rule restriction on the value of the Fermi liquid coefficients based on the Pauli exclusion principle $^{34,18}$. These restrictions will not be particularly important for our present discussion.

This relation (Eq. 91) between the $f_{1}$ coefficient, the effective mass and the bare mass is an extremely important result. This tells us that within Fermi liquid theory, whenever the effective mass is renormalized from the bare mass, then in order to have a consistent theory one must also include an interaction of the $f_{1}$ type. In other words, if we think of the effective mass of the quasiparticle becoming different from the bare mass in some sort of renormalized theory ${ }^{33}$, then the renormalization procedure should also generate an $f_{1}$ type interaction. In section 3.9 above, we had trouble renormalizing the composite fermion mass in a consistent way. The reason for this trouble was that we neglected to consider the possibility of an $f_{1}$ interaction. By including such an interaction term in sections 4.2 and 4.3 below, we will be able to fix this problem.

\subsubsection{Boltzmann Transport}

We now consider the derivation of a transport equation. The quasiparticle en$\operatorname{ergy} \tilde{\epsilon}$ can be used as an effective one particle Hamiltonian to derive a LandauBoltzmann transport ${ }^{k}$ equation $^{29,30}$

$$
\frac{\partial n}{\partial t}+\nabla_{\mathbf{r}} n \cdot \nabla_{\mathbf{p}} \tilde{\epsilon}-\nabla_{\mathbf{p}} n \cdot \nabla_{\mathbf{r}} \tilde{\epsilon}+\mathbf{F} \cdot \nabla_{\mathbf{p}} n=\left(\frac{\partial n}{\partial t}\right)_{\text {coll }}
$$

where $\mathbf{F}(\mathbf{r})$ is an applied force and the right hand side is a collision term.

\footnotetext{
${ }^{k}$ The Boltzmann equation is nothing more than conservation of particle number in phase space. The current in the real space direction is $\mathbf{j}_{r}=n \mathbf{u}=n \frac{d \mathbf{r}}{d t}=n \nabla_{\mathbf{p}} \tilde{\epsilon}$. Analogously the current in the momentum space direction is $\mathbf{j}_{p}=n \frac{d \mathbf{p}}{d t}=n\left(\mathbf{F}-\nabla_{\mathbf{r}} \tilde{\epsilon}\right)$. Thus we obtain the Boltzmann equation by conserving particle number $\nabla_{\mathbf{p}} \cdot \mathbf{j}_{p}+\nabla_{\mathbf{r}} \cdot \mathbf{j}_{r}+\frac{\partial n}{\partial t}=0$.
} 
We now consider applying a uniform magnetic field $B$ to the system as well as a weak perturbing electric field $\mathbf{E}$ at wavevector $q$ and frequency $\omega$. We will maintain the convention that $\mathbf{q} \| \hat{\mathbf{x}}$ such that all perturbations are proportional to $e^{i q x-i \omega t}$. With some effort, the Boltzmann equation can be then be linearized and written in terms of the small fluctuation of the Fermi surface $\operatorname{as}^{35,29}$

$$
-i \omega \nu(\theta)+\left(i q v_{\mathrm{F}}^{*} \cos (\theta)-\omega_{\mathrm{c}}^{*} \frac{\partial}{\partial \theta}\right)\left[\nu(\theta)+\delta \epsilon_{1}(\theta)\right]=-e \mathbf{E} \cdot \hat{\mathbf{n}}(\theta)+I(\theta)
$$

where

$$
\delta \epsilon_{1}(\theta)=\frac{m^{*}}{(2 \pi \hbar)^{2}} \int d \theta^{\prime} f\left(\theta-\theta^{\prime}\right) \nu\left(\theta^{\prime}\right),
$$

where $\hat{\mathbf{n}}(\theta)=(\cos \theta, \sin \theta)$, with $\omega_{\mathrm{c}}^{*}=\frac{e B}{m^{*} c}$, the mass renormalized cyclotron frequency, and $I(\theta)$ a scattering term whose form will be discussed in the next section. In the case of the Chern-Simons Fermi liquid, we should use the effective cyclotron frequency $\Delta \omega_{\mathrm{c}}^{*}=\frac{e \Delta B}{m^{*} c}$ seen by the transformed fermions.

\subsubsection{Scattering}

As a model of scattering, one might hope to use the phenomenological relaxation form

$$
I(\theta)=-\tau^{-1} \nu(\theta)
$$

Although this is very simple, and clearly corresponds to an analytic continuation of $\omega \rightarrow \omega+\frac{i}{\tau}$ in Eq. 93, we will see that this simple form is not allowed.

If we apply the operator $\int_{0}^{2 \pi} d \theta$ to both sides of the kinetic equation (Eq. 93), with some algebra we obtain

$$
\frac{4 \pi^{2} \hbar^{2}}{p_{\mathrm{F}}}\left(i \omega \delta n-i q j_{x}\right)=\int_{0}^{2 \pi} d \theta I(\theta)
$$

Thus, in order to assure local current conservation $\omega \delta n=q j_{x}$, we must have a "current conserving" scattering term ${ }^{l}$ that satisfies $\int_{0}^{2 \pi} I(\theta) d \theta=0$. Thus, the simplest model of scattering that one may consider is given by ${ }^{36}$

$$
I(\theta)=-\frac{1}{\tau}\left(\nu(\theta)-\frac{1}{2 \pi} \int_{0}^{2 \pi} d \theta \nu(\theta)\right)
$$

\footnotetext{
${ }^{l}$ Scattering due to quasi-particle collisions would be subject to conservation of momentum density too. This would require also $\int_{0}^{2 \pi} d \theta \cos (\theta) I(\theta)=0$.
} 
The use of such a density preserving scattering term has been emphasized in Ref. 37. However, we will see below that this current conservation correction to the scattering term typically has a pretty small effect, and sometimes has exactly no effect.

\subsubsection{Results of Conventional Fermi Liquid Theory}

In this section, we quote results regarding the calculation of conductivities from the Boltzmann equation. The general method of calculation is to impose some electric field $\mathbf{E}(q, \omega)$ in Eq. 93 and solve for the resulting fluctuation of the Fermi surface $\nu(q, \omega)$. Once this is obtained, the current is given by Eqs. 83,86 , and 87 , which enables us to extract the conductivity. In appendix A we have shown such a calculation for the case of a noninteracting Fermi system (i.e., all $f_{l}=0$ ) in zero magnetic field. Reasonably simple results can also be obtained for free fermions in a magnetic field ${ }^{38,16,14}$.

The inclusion of nonzero Fermi liquid coefficients in general makes the solution of the Boltzmann equation more difficult. However, the effects of $f_{0}$ and $f_{1}$, as well as the effects of the scattering model discussed above (Eq. 97) can be treated explicitly without re-solving the full Boltzmann equation ${ }^{39}$. These "tricks" for solving more complicated Boltzmann equations will be described here and the proofs are left for the adventurous readers.

For treating $f_{0}$, we relate the resistivity matrix of an arbitrary system to the resistivity matrix of a system that is the same except that the zero ${ }^{t h}$ Landau coefficient $f_{0}$ has been artificially set to zero. We call the resistivity of this artificial system $\rho^{f_{0}=0}$, and we have the relation

$$
\rho=\rho^{f_{0}=0}+\frac{i f_{0} q^{2}}{\omega e^{2}}\left(\begin{array}{ll}
1 & 0 \\
0 & 0
\end{array}\right) .
$$

In terms of a polarization matrix (just by applying factors of $T$ ), we might write the analogous

$$
\Pi^{-1}=\Pi_{f_{0}=0}^{-1}+\left(\begin{array}{cc}
f_{0} & 0 \\
0 & 0
\end{array}\right) .
$$

The form of this result should be clear. This is just an RPA-like treatment of the $f_{0}$ interaction which is a density-density interaction (like the Coulomb interaction) and therefore enters only in one component of the inverse response (compare Eqs. 48 and 45).

Similarly, for $f_{1}$, we can relate the resistivity of an arbitrary system to the resistivity of an artificial system for which the first Landau coefficient $f_{1}$ has

been set to zero and the particle mass is set equal to $m^{*}$. We call the resistivity 
of the artificial system $\rho_{f_{1}=0}^{*}$ and derive the result ${ }^{m}$

$$
\rho=\rho_{f_{1}=0}^{*}+\left[i \omega \frac{f_{1} m^{*}}{2 \pi \hbar^{2}} \frac{m_{\mathrm{b}}}{e^{2} n_{\mathrm{e}}}\right] I
$$

with $I$ the identity matrix. In terms of a polarization again we have

$$
\Pi^{-1}=\left[\Pi_{f 1=0}^{*}\right]^{-1}+\frac{m^{*}-m_{\mathrm{b}}}{n_{\mathrm{e}} e^{2}}\left(\begin{array}{cc}
\frac{\omega^{2}}{q^{2}} & 0 \\
0 & -1
\end{array}\right)
$$

The ${ }^{*}$ here reminds us that this polarization should be calculated using the effective mass (but with $f_{1}=0$ ). The form of this equation is similarly a RPA-like treatment of the current-current interaction $f_{1}$.

We can also consider the effect of a scattering term. We recall that the simplest scattering model (Eq. 95) does not conserve current. However, the solution of such a nonphysical model is obtained very simply by analytic continuation $\left(\omega \rightarrow \omega+\frac{i}{\tau}\right)$ of the resistivity of a system without scattering (this should be obvious from Eq. 93). The solution to the current conserving model (Eq. 97) can also be obtained in a similar manner. The additional term in the current conserving model has the effect of an $f_{0}$ coefficient yielding the result

$$
\rho(\omega)=\rho^{f_{0}=0, \tau=\infty}\left(\omega+\frac{i}{\tau}\right)+\frac{i \kappa q^{2}}{\left(\omega+\frac{i}{\tau}\right) e^{2}}\left(\begin{array}{ll}
1 & 0 \\
0 & 0
\end{array}\right) .
$$

with

$$
\kappa=\left(1+\frac{i}{\omega \tau}\right) f_{0}+\frac{2 \pi \hbar^{2}}{m^{*}} \frac{i}{\omega \tau} .
$$

where $\rho^{f_{0}=0, \tau=\infty}(\omega)$ is the resistance of a system identical to our actual system, but without scattering $(\tau=\infty)$ and with the zeroth Fermi liquid coefficient set to zero. Once this resistance is calculated for real $\omega$, it must be analytically continued to $\omega+\frac{i}{\tau}$. We note that only the $\rho_{x x}$ term of the resistivity matrix distinguishes between the current conserving and the non-current conserving models of scattering.

Thus, we have here several rules for relating resistivities of certain systems to resistivities of simpler systems. As a simple example of using some of these results, we consider a system of particles in zero magnetic field with a Landau interaction function that has nonzero $f_{0}$ and $f_{1}$ coefficients (but with all other coefficients zero) and with the current conserving scattering term (Eq. 97). We will focus on obtaining $\rho_{y y}$ since this is what will be most relevant for the

\footnotetext{
${ }^{m}$ In the appendix of Ref. 16 this is derived in an extremely complicated way. A much cleaner, and more general, RPA-like derivation ${ }^{39}$ of this result can also be performed.
} 
Chern-Simons problem (See Eq. 31). From Eqs. 98 and 102 it is clear that $f_{0}$ and the current conservation correction has no effect on $\rho_{y y}$. Thus, we can obtain $\rho_{y y}$ for this model by simply analytically continuing the results of Eq. 100 via $\omega \rightarrow \omega+\frac{i}{\tau}$. Using Eq. A.7 for the Boltzmann conductivity of free fermions with Eq. 100 then yields the result (recall $F_{1}=\frac{m^{*} f_{1}}{2 \pi \hbar^{2}}$ )

$$
\rho_{y y}=i \frac{2 \pi \hbar}{e^{2}} \frac{q}{k_{\mathrm{F}}}\left[-\frac{1}{\Omega}\left(1-\left[1-\frac{1}{\Omega^{2}}\right]^{1 / 2}\right)^{-1}+2 \Omega\left(\frac{F_{1}}{1+F_{1}}\right)\right] .
$$

with $\Omega=\left(\omega+\frac{i}{\tau}\right) /\left(q v_{\mathrm{F}}^{*}\right)$. It is worth noting that the form of the resistivity is given by $\rho_{y y}=q G_{y y}(\Omega)$ with $G_{y y}$ some function. Without scattering, $\rho_{x x}$ takes a similar form.

We might guess at this point that we can use this resistivity as $\rho_{y y}^{\mathrm{CF}}$ in Eq. 31 to obtain the electrical conductivity of the even denominator states at $\nu=\frac{1}{2 m}$. Indeed, this is exactly what we did in obtaining Eqs. 35 and 36 . We will now examine in more detail exactly what this prescription amounts to.

\subsection{Landau-Silin Chern-Simons Theory}

Clearly, the above local energy functional (Eq. 77) can not properly represent a long range interaction. More generally, an extension of Fermi-liquid theory was developed by Silin ${ }^{29,30,40}$ that separates out the Hartree part of the long range interaction and uses the Landau interaction function $f$ to represent the short range part of the interaction. In Landau and Silin's original work ${ }^{40}$, this approach was used to describe electrons with long range Coulomb interactions. The prescription they used was to calculated the conductivity (or polarization $\Pi^{v}=T^{-1} \sigma T^{-1}$ ) using the Boltzmann equation with effective mass $m^{*}$ and interaction function $f$. The long range Coulomb interaction is then added on last via Eq. 48 (which is equivalent to Thomas-Fermi, or self-consistent Hartree screening). The justification for this prescription is simply that the fermions must respond to the externally applied force as well as the internally induced force (the self-consistent Hartree piece). This approach can be justified in an explicit perturbation theory ${ }^{29,30}$ for the long wavelength low frequency limit, and has also been quite successful for treating stronger interactions, although it has not been rigorously justified.

In the case of the Chern-Simons Fermi liquid, a similar prescription can be used $^{16}$. Here, we should calculate the Composite fermion conductivity $\sigma^{\mathrm{CF}}$ (or polarization $\Pi=T^{-1} \sigma^{\mathrm{CF}} T^{-1}$ ) using the Boltzmann equation with an effective mass $m^{*}$ and interaction function $f$. The Chern-Simons interaction is then treated at self-consistent Hartree level to yield an electron resistivity given by 
$\rho=\rho^{\mathrm{CF}}+\rho^{\mathrm{CS}}$ (Eq. 30). The full electromagnetic response can similarly be calculated by using Eq. 64. Thus we now have a full prescription for calculating responses for our Chern-Simons system through a Landau-Silin Boltzmann framework. (Note that below in section 5 we will see that this prescription neglects important magnetization terms. However, for now we will not be concerned with these effects, and we will see later that our neglect is justified at least so long as we are concerned with calculating only the longitudinal responses $K_{00}$ or $\sigma_{x x}$.)

We recall that in section 3.9 above ${ }^{14,16}$, there was difficulty in obtaining a response that both satisfied the sum rules (Kohn's theorem and the $f$-sum rule) and also had low energy excitations on the right (interaction) energy scale. We found that either we used the bare mass $m_{\mathrm{b}}$ in our calculation and we ended up with low energy excitations with energy scale set by $m_{\mathrm{b}}$ (instead of $m^{*}$ ), or we used an effective mass $m^{*}$ in our calculation and we found that the Kohn mode occurred at a frequency set by $m^{*}$ instead of a frequency set by $m_{\mathrm{b}}$ as is required by Galilean invariance. As mentioned above in section 4.1.2 the reason we had this problem is that renormalization of the mass must imply a nonzero Fermi liquid interaction coefficient $f_{1}$ (See Eq. 91). In this Landau-Silin approach it is very easy to include such an interaction term in our calculation to restore Galilean invariance (satisfying the sum rules) while keeping the low energy excitations on the scale of the effective mass. Thus, to phenomenologically renormalize the energy scale in this framework we need only change $f_{1}$ and $m^{*}$ together.

In terms of the response function $K$, we write

$$
\begin{aligned}
K^{-1} & =\left[\Pi_{f_{1}=0}^{*}\right]^{-1}+\mathcal{F}_{1}+V+C \\
\mathcal{F}_{1} & =\frac{\left(m^{*}-m_{\mathrm{b}}\right)}{n_{\mathrm{e}} e^{2}}\left(\begin{array}{cc}
\frac{\omega^{2}}{q^{2}} & 0 \\
0 & -1
\end{array}\right) .
\end{aligned}
$$

Note that the addition of the $\mathcal{F}_{1}$ term is the same as in Eq. 101. The LandauSilin result is then achieved by approximating $\Pi_{f_{1}=0}^{*}=T^{-1} \sigma T^{-1}$ as the Boltzmann response of fermions of mass $m^{*}$ in the mean magnetic field $\Delta B$. If we assume all of the other Fermi liquid coefficients are zero $\left(f_{n}=0\right.$ for $\left.n \neq 1\right)$, then $\Pi^{*}$ represents the response of free fermions. The Boltzmann response of free fermions is calculated explicitly in appendix $\mathrm{A}$ for the case of $\Delta B=0$ which corresponds to the case of $\nu=\frac{1}{2 m}$. Within this framework, it is very easy to include a phenomenological scattering time as discussed above. This Boltzmann approach (neglecting all other Fermi liquid coefficients) was called a "Modified Semiclassical" approximation in Ref. 16. In section 8.1 this type of approximation is used to make predictions for the results of surface acoustic 
wave experiments.

\subsection{Modified RPA (MRPA)}

The Landau-Silin approach discussed above depends on the Boltzmann equation for calculating responses. As discussed in appendix A, the Boltzmann equation misses some important features of the response function, such as the diamagnetic term of the transverse response. Furthermore, the Boltzmann equation, being semiclassical, does not know about the incompressibility of the system when an integer number of Landau levels are filled. For this reason, we would like to define an approximation based on this Landau-Silin approach that has some of these features properly included (at least in some approximation).

In Ref. 16 a Modified RPA (MRPA) was constructed that attempts to include these effects. The MRPA is obtained by setting $\Pi^{*}$ in Eq. 105 equal to the response $K^{0 *}$ of a system of noninteracting fermions of mass $m^{*}$ in the mean magnetic field $\Delta B$. The response function thus calculated (using $\Pi^{*}=K^{0 *}$ and Eq. 105) will be called $K^{\mathrm{MRPA}}$. Thus, we have

$$
\left[K^{\mathrm{MRPA}}\right]^{-1}=\left[K_{0}^{*}\right]^{-1}+\mathcal{F}_{1}+V+C
$$

Results of such MRPA calculations for the Jain fractions $\nu=\frac{p}{2 m p+1}$ are shown in Refs. 16 and 41. A figure from Ref. 16 is also reproduced in the chapter by Willett in this volume.

At filling fraction $\nu=\frac{1}{2 m}$ there is zero effective field so the response $K^{0 *}$ is diagonal. We can then use the MRPA prescription (Eq. 107) to obtain

$$
K_{00}=\frac{1}{\left(\frac{2 \pi \tilde{\phi}}{q}\right)^{2}\left(\left[K_{11}^{0}\right]^{-1}+\frac{m^{*}-m_{\mathrm{b}}}{n}\right)^{-1}+\left(\left[K_{00}^{0}\right]^{-1}-\frac{m^{*}-m_{\mathrm{b}}}{n} \frac{\omega^{2}}{q^{2}}\right)+v(q)}
$$

When $m_{\mathrm{b}}=m^{*}$ this reduces to the RPA result (Eq. 67). We also note that in the limit of $\omega \ll q v_{\mathrm{F}}^{*}$, this reduces to the form of the RPA result (Eq. 68) except that all factors of $m_{\mathrm{b}}$ are replaced by factors of $m^{*}$. Thus, as with the RPA result, this response also has an overdamped mode at $\omega \sim q^{3} v(q)$ that will result in infrared divergences when we try to calculate further corrections in section 6.3 below. In Fig. 2 the excitation spectrum for $\nu=\frac{1}{2}$ is shown for such an MRPA calculation with $m^{*}=10 m_{\mathrm{b}}$. The energy scale in that figure is the renormalized cyclotron frequency, so on that scale the bare cyclotron mode is off of the top of the graph.

Comparisons of results of exact diagonalizations of small systems projected to the Lowest landau level to results of $K_{00}$ calculated in the MRPA were quite 

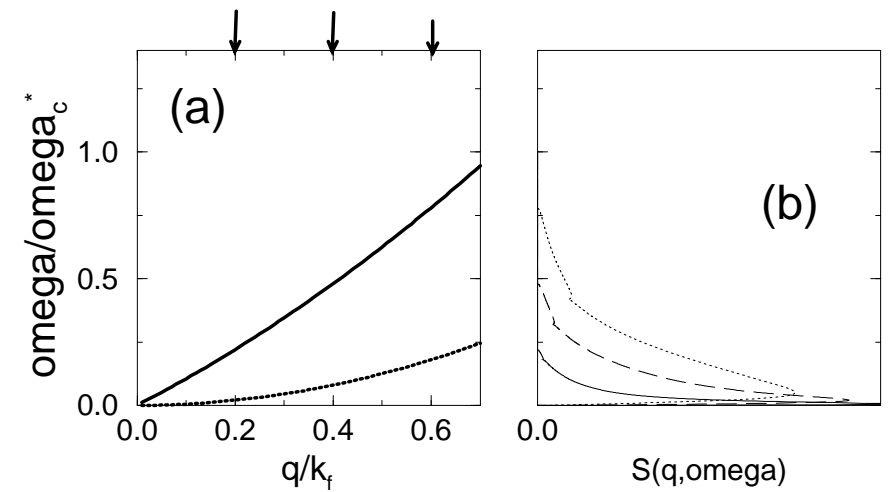

Figure 2: Excitation spectrum of the $\nu=\frac{1}{2}$ state in MRPA for $m^{*}=10 m_{\mathrm{b}}$ Note that the energy scale is in units of the renormalized cyclotron frequency $\omega_{\mathrm{c}}^{*}=$ $\frac{e B}{m^{*} c}$. In (a) the solid line is the edge of the low energy continuum of quasiparticles, the dotted line is the location of the peak in the weight (the maximum of the structure factor) of the low energy continuum $\left(\omega \sim q^{3} v(q)\right)$. The cyclotron mode is off the top of the graph. In (b) the structure factor $S(q, \omega)$ is shown explicitly for three different wavevectors $q / k_{\mathrm{F}}=.2$ (solid), .4. (dashed), and .6 (dotted). The small cusp in the structure factor is a reflection of a similar cusp that occurs in $K^{0}$ for noninteracting fermions. In comparison to the RPA (See fig. 1) the weight is pushed to even lower relative frequency. Here we have used $E_{\mathrm{c}} /\left(\hbar \omega_{\mathrm{c}}^{*}\right)=5$ which is a reasonable theoretical estimate ${ }^{57,42}$. 
favorable $^{41}$ for the low energy excitations at $\nu=\frac{p}{2 m p+1}$ for small $p$. Similar agreement ${ }^{42}$ was found for the low energy modes of small systems at $\nu=1 / 2$.

To summarize this section, we have found that by framing the ChernSimons theory in a Landau-Silin Fermi liquid description, we have been able to renormalize the mass of the quasiparticle in a consistent manner. Thus, at least phenomenologically, we can have the low energy excitations on the scale of the interaction strength a while maintaining the cyclotron mode at the bare cyclotron frequency. Perhaps more importantly, we have a put the ChernSimons theory in the versatile language of the Landau Fermi liquid. Despite these successes, and despite the numerical successes in the calculation of $K_{00}$, we will show in the next section that the (M)RPA does not properly represent the other elements of the response matrix $\left(K_{01}, K_{10}\right.$, and $\left.K_{11}\right)$ in the limit of $m_{\mathrm{b}} \rightarrow 0$.

\section{Magnetization and $\mathrm{M}^{2} \mathrm{RPA}$}

We now turn to consider the limit of small band mass $m_{\mathrm{b}}$ (or equivalently large magnetic field $B$ ) so that the cyclotron frequency $\frac{e B}{m_{\mathrm{b}} c}$ is extremely large. In particular, in section 5.1 we focus on the zero frequency, finite wavevector electromagnetic response in this limit. The fact that, in this limit, the electronic ground state and low energy excitations are constrained to the lowest Landau level, leads to certain features of the electronic response which are not properly represented in approximation schemes such as the mean field or the (M)RPA. We note that this problem occurs in the Chern-Simons theory even when gauge-field fluctuations are not infra-red singular ${ }^{a}$ (See section 6.3 below).

We discuss in this section an approach ${ }^{17,18}$ based on a phenomenological separation of the current into a magnetization current associated with the cyclotron motion of electrons and a transport current associated with the guiding center motion. This separation, discussed in section 5.2 is achieved by attaching a magnetization $\mu_{\mathrm{M}}$ to each particle. This magnetization originates from the electrons' orbital motion and is unrelated to the spin (we have assumed spinless electrons throughout this paper). In the limit $m_{\mathrm{b}} \rightarrow 0$, the magnetization $\mu_{\mathrm{M}}$ is given by the Bohr magneton ${ }^{b}$

$$
\mu_{\mathrm{b}}=\frac{e \hbar}{2 m_{\mathrm{b}} c}
$$

\footnotetext{
${ }^{a}$ For example, if the electron-electron repulsion falls off more slowly than $1 / r$ there should be no infra-red divergences in the effective mass.

${ }^{b}$ Here it is convenient that the subscript b can stand for 'Bohr' or 'bare' to remind us that it is the bare mass that enters this expression.
} 
such that $\mu_{\mathrm{b}} B$ represents the noninteracting ground state kinetic energy $\frac{1}{2} \hbar \omega_{\mathrm{c}}$. The proposed separation procedure combined with approximations similar to those made in the MRPA, results in an approximation we call the $\mathrm{M}^{2} \mathrm{RPA}$, discussed in section 5.3, that yields a response functions that correctly describes the $m_{\mathrm{b}} \rightarrow 0$ limit. In section 5.4 we then revisit Fermi liquid theory and see how we can fit the $\mathrm{M}^{2} \mathrm{RPA}$ into the framework of a Landau theory. We note that the Lowest Landau level approach of Shankar and Murthy ${ }^{49}$ explicitly demonstrates this magnetization attachment.

\subsection{Zero Frequency Response}

In this section we shall examine the form of the zero frequency finite wavevector response in the high magnetic field (or $m_{\mathrm{b}} \rightarrow 0$ ) limit. An acceptable approximation for calculating the response of the $\nu=\frac{1}{2 m}$ state must make correct predictions in this limit. We will show below that the usual Chern-Simons approaches do not do this.

Consider the $\nu=\frac{1}{2 m}$ state in the limit $m_{\mathrm{b}} \rightarrow 0$. In this limit the cyclotron gap $e B /\left(m_{\mathrm{b}} c\right)$ between Landau levels becomes large so we expect such a system to be restricted to the lowest Landau level. If we apply a weak external static scalar potential at wavevector $q$ to the system, the resulting state should remain in the lowest Landau level so the induced density fluctuation should depend only on the interaction strength, and not on the bare mass $m_{\mathrm{b}}$. Thus, $K_{00}$, the density-density response, should be independent of the bare mass in this limit (or more properly, should scale as $\left(m_{\mathrm{b}}\right)^{0}$ plus $\mathcal{O}\left(m_{\mathrm{b}}\right)$ corrections). However, the resulting density inhomogeneity will yield a transverse current called the magnetization current, given by (here and below the speed of light $c=1$ ) $\mathbf{j}_{\text {mag }}=\hat{\mathbf{z}} \times \nabla \mathbf{M}$ with $\mathbf{M}$ the magnetization density. For noninteracting particles in the lowest Landau level, the kinetic energy density is $E \equiv \mathbf{M} \cdot \mathbf{B}=\frac{1}{2} \hbar \omega_{\mathrm{c}} n_{\mathrm{e}}$ so that the magnetization per particle is $|\mathbf{M}| / n_{\mathrm{e}}=\mu_{\mathrm{b}}$, the Bohr magneton. More generally, when interactions are taken into account, we let the magnetization per particle be given by a quantity $\mu_{\mathrm{M}}$ which must become $\mu_{\mathrm{b}}$ in the $m_{\mathrm{b}} \rightarrow 0$ limit where the system becomes projected to the Lowest Landau level. We can thus write the magnetization current as ${ }^{c}$

$$
\mathbf{j}_{\mathrm{mag}}=\mu_{\mathrm{M}}(\hat{\mathbf{z}} \times \nabla n)
$$

with $n(\mathbf{r})$ the local electron density. The physical interpretation of this magnetization current as follows. Each particle in the lowest Landau level can be

${ }^{c}$ When projected to the lowest Landau level, the projected current and density operators satisfy $P \mathbf{j} P=\mu_{\mathrm{b}}(\hat{\mathbf{z}} \times \nabla P n P)$ where $P$ is the projection operator. In other words, for projected states, all of the current is magnetization current. 
thought of as a particle in a cyclotron orbit. When the density of particles is uniform, the local currents of all of these orbits cancel and there is no net current in the system. However, when there is a density inhomogeneity, these local currents do not quite cancel and a net magnetization current results. Note that this magnetization current associated with density gradients can be modeled by imagining that a small magnetization $\mu_{\mathrm{M}}$ (equivalent to a current loop) is attached to each quasiparticle.

Using Eq. 110 we see that in the limit $m_{\mathrm{b}} \rightarrow 0$, when we apply the weak static scalar potential $A_{0}^{\text {ext }}(q)$ to the system and we look at the leading current response we find a magnetization current $\mu_{\mathrm{b}} \hat{\mathbf{z}} \times i \mathbf{q} K_{00} A_{0}^{\text {ext }}$. Thus, if $q$ is finite we expect

$$
\lim _{m_{\mathrm{b}} \rightarrow 0} K_{10} / K_{00}=i q \mu_{\mathrm{b}}
$$

This result is not ${ }^{d}$ contained in works based on the Chern-Simons approach previous to that of Ref. 17 .

We can also consider applying a weak external transverse vector potential $A_{1}^{\text {ext }}$ at wavevector $q$ and zero frequency. This transverse field generates a magnetic field $\delta B=i q A_{1}$ at wavevector $q$. The variation in the total magnetic field $B(\mathbf{r})=B_{1 / 2}+\delta B(\mathbf{r})$ will make the kinetic energy $\frac{1}{2} \hbar \omega_{\mathrm{c}}(\mathbf{r})=\mu_{\mathrm{b}} B(\mathbf{r})$ positionally dependent thus attracting electrons to the regions of minimal magnetic field when $m_{\mathrm{b}} \rightarrow 0$. This attraction is not modeled in the Chern-Simons fermion picture at the mean field or (M)RPA level.

Formally, if the applied variation in magnetic field generates a density fluctuation $j_{0}(q)$, we can write the energy cost as $\delta E=j_{0}(\delta B) \mu_{\mathrm{M}}+\frac{1}{2} K_{00} j_{0}^{2}$ where $K_{00}$ is independent of $m_{\mathrm{b}}$ as discussed above. Here, $j_{0}(\delta B) \mu_{\mathrm{M}}$ is just the change in local cyclotron energy which can be thought of as an effective scalar potential for the fermions. This term would occur quite naturally if we were to imagine that a magnetization $\mu_{\mathrm{M}}$ were attached to each fermion. The term $K_{00} j_{0}^{2}$ is due to the Coulomb interactions within the lowest Landau level. Again note that $\mu_{\mathrm{M}}$ must become $\mu_{\mathrm{b}}$ in the $m_{\mathrm{b}} \rightarrow 0$ limit, but more generally can include pieces on the interaction scale. Minimizing the energy $\delta E$ with respect to $j_{0}$ yields the density $j_{0}=-(\delta B) \mu_{m} K_{00}=-i q \mu_{\mathrm{M}} K_{00} A_{1}$ from which we conclude that that the leading term of $K_{01}$ is given by $i q \mu_{\mathrm{M}} K_{00}$ (in accordance with the symmetry requirement of the matrix $K$ ).

Finally, once we have determined the density fluctuation due to this local magnetic field fluctuation, we again realize that this density fluctuation results

\footnotetext{
${ }^{d}$ In the MRPA approach it is clear that this ratio cannot be determined by the bare mass since the low energy physics is controlled by $m^{*}$ (the whole purpose of the MRPA was to get $m_{\mathrm{b}}$ out of the low energy physics). On the other hand in RPA this ratio is indeed on the scale of $m_{\mathrm{b}}$ (since that is the only scale in the problem), but it can be shown that the coefficient out front is incorrect (not to mention the other problems with RPA).
} 
in a magnetization current, so that we have a leading piece of $K_{11}$ given by $K_{00} q^{2} \mu_{\mathrm{M}}{ }^{2}$.

\subsection{Binding Magnetization to Composite Fermions}

As suggested by the above discussion, the necessary correction to the composite fermion picture involves attaching a magnetization $\mu_{\mathrm{M}}$ to each composite fermion so that it properly represents a particle in the lowest Landau level. Attaching magnetization to each particle can also be interpreted as attaching a current loop to each particle associated with the electrons' cyclotron motion. Thus the total current would include both a piece from the motion of the particle-currentloop composite and a piece from the current loop itself. To this end, we define a transport current ${ }^{e}$

$$
\mathbf{j}_{\text {trans }}=\mathbf{j}_{\text {total }}-\mathbf{j}_{\text {mag }}
$$

which is the current of magnetized gauge transformed fermions, whereas the magnetization current, as discussed above (see Eq. 110) is the current associated with the attached current loops.

In addition, particles bound to magnetization should experience an effective potential associated with any local changes in the magnetic field. Thus we define the effective scalar potential

$$
A_{0}^{\mathrm{eff}}=A_{0}+\mu_{\mathrm{M}} \delta B .
$$

This interaction of the bound magnetization with the magnetic field should be thought of as the effective potential associated with the local change in the cyclotron energy.

If we keep the conventions that all perturbations are applied with $\mathbf{q} \| \hat{\mathbf{x}}$, and use the Coulomb gauge again, we can rewrite Eqns. 112 and 113 as

$$
\begin{aligned}
j_{\text {total }} & =M j_{\text {trans }} \\
A_{\text {eff }} & =M^{\dagger} A
\end{aligned}
$$

where

$$
M=\left[\begin{array}{cc}
1 & 0 \\
i q \mu_{M} & 1
\end{array}\right] .
$$

In these equations, all currents are written as two vectors $\left(j_{0}, j_{y}\right)$ and vector potentials are written as two vectors $\left(A_{0}, A_{y}\right)$. The matrix $M$ should be

${ }^{e}$ The division into $\mathbf{j}_{\text {trans }}$ and $\mathbf{j}_{\text {mag }}$ has some degree of arbitrariness. Note that the definitions in the present paper allow for a nonzero transverse component of $\mathbf{j}_{\text {trans }}$ in equilibrium for an inhomogeneous interacting electron system. 
thought of as an operator that attaches magnetization. As discussed above, in the limit $m_{\mathrm{b}} \rightarrow 0$, we must have $\mu_{\mathrm{M}} \rightarrow \mu_{\mathrm{b}}$ in the matrix $M$, but more generally we can allow corrections on the interaction scale. In the rest of this section, however, we will focus on the $m_{\mathrm{b}} \rightarrow 0$ limit and consider $\mu_{\mathrm{M}}=\mu_{\mathrm{b}}$.

\subsection{Magnetized Modified RPA $\left(M^{2} R P A\right)$}

As discussed above, the (M)RPA approach does not properly model the magnetization effects discussed in section 5.1. This error is presumably due to the fact that when we take the mean field solution as a starting point for a perturbation theory for the Chern-Simons fermions, we lose the fact that the original electrons travel in local cyclotron orbits. In the approach discussed here $^{17}$, we will recover this physics by artificially attaching magnetization to each particle by hand. This attachment is not an exact transformation, but is rather a way of modeling behavior that is lost when we take the mean field as a starting point. However, as we will see below, within a Landau-Fermi liquid theory picture, this attachment seems to give the correct quasiparticles for the system.

The magnetized particles have the same interactions (Both Chern-Simon $C$ and Coulomb $V$ ) as the particles in the traditional Chern-Simons fermion picture. However, here, the magnetized fermions now respond to the effective potential and the motion of these magnetized fermions yields only the transport current response. We thus define a matrix $\tilde{K}$ to be the transport current response ${ }^{f}$ of the electrons to the external effective potential. In other words,

$$
K=M \tilde{K} M^{\dagger} .
$$

The 'Magnetized Modified RPA' or $\mathrm{M}^{2} \mathrm{RPA}$ is then defined by setting $\tilde{K}$ equal to $K^{\mathrm{MRPA}}$. Thus we have

$$
K^{\mathrm{M}^{2} \mathrm{RPA}}=M K^{\mathrm{MRPA}} M^{\dagger}=M\left(\left[K^{0 *}\right]^{-1}+\mathcal{F}_{1}+C+V\right)^{-1} M^{\dagger} .
$$

with $\mathcal{F}_{1}$ defined by Eq. 106 . It should be noted that

$$
K_{00}^{\mathrm{M} 2 \mathrm{RPA}}=K_{00}^{\mathrm{MRPA}}
$$

and therefore the exact diagonalizations ${ }^{41,42}$ that agreed well with calculations of $K_{00}$ in the MRPA agree equally well with predictions of the $\mathrm{M}^{2} \mathrm{RPA}$. However, the MRPA and $\mathrm{M}^{2} \mathrm{RPA}$ differ at finite $q$ in their predictions for the other elements of the matrix $K$. For example,

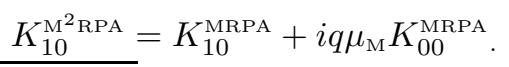

\footnotetext{
${ }^{f}$ The reader is cautioned that this is not the same as the $\tilde{K}$ defined in $\operatorname{HLR}^{14}$, which is what we call $\Pi$.
} 
It should be noted however, that all finite $q$ experimental tests ${ }^{24}$ of the ChernSimons theory to date have measured only $K_{00}$ and therefore do not distinguish between the MRPA and the $\mathrm{M}^{2} \mathrm{RPA}$. As required, in the limit $m_{\mathrm{b}} \rightarrow 0$, the $\mathrm{M}^{2} \mathrm{RPA}$ correctly describes the static response properties described above. For example, Eq. 120 clearly satisfies Eq. 111.

We note that we expect that the $\mathrm{M}^{2} \mathrm{RPA}$, in addition to describing the $\nu=\frac{1}{2 m}$ Fermi liquid states, should properly describe the magnetization effects for the Jain series of quantized states $\nu=\frac{p}{2 m p+1}$ at least for small $p$.

\subsection{Fitting into Fermi Liquid Theory}

We now turn to discuss how the $\mathrm{M}^{2} \mathrm{RPA}$ fits into the general picture of a Fermi liquid theory of the $\nu=\frac{1}{2 m}$ state. In essence, we will show that $\mathrm{M}^{2} \mathrm{RPA}$ roughly amounts to adopting the Fermi liquid picture discussed above in section 4 as describing the dynamics of magnetized composite fermion quasiparticles rather

than unmagnetized ones. We recall that in conventional Fermi liquid theory the effective mass of the quasiparticle can be highly renormalized from the effective mass of the bare particle. (In the case of ${ }^{3} \mathrm{He}$, the quasiparticle effective mass is approximately three times the bare mass, such that the quasiparticle is quite different from the original particle). In our composite fermion system, our quasiparticle will not only have a renormalized mass, but also a renormalized magnetization.

For the Chern-Simons theory, in addition to separating the long ranged part of the interaction $(C+V)$, for the magnetized fermions, further separation should be carried out to remove the magnetization effects. To this end we define a response function $\tilde{\Pi}$ by (See Eq. 64 for the definition of $\Pi$ )

$$
\Pi=M \tilde{\Pi} M^{\dagger} .
$$

and correspondingly we define a conductivity

$$
\tilde{\Pi}=T^{-1} \tilde{\sigma}^{\mathrm{CF}} T^{-1}
$$

By definition, II relates the transport current of the magnetized quasiparticles to the effective total vector potential, including both external and internally induced contributions (See Eqns. 114, 115 and 62). For the ChernSimons system it is now $\tilde{\Pi}=T^{-1} \tilde{\sigma}^{\mathrm{CF}} T^{-1}$ which we claim is given by a LandauBoltzmann equation describing the dynamics of quasiparticles with the finite effective mass $m^{*}$ interacting via a residual short ranged interaction $f$.

We now have a prescription for calculating the response $K$ of the ChernSimons Fermi liquid given the effective mass $m^{*}$ and the interaction function $f(\theta)$. To reiterate, the prescription is to solve the Boltzmann equation (Eq. 93) 
to obtain the magnetized composite fermion conductivity $\tilde{\sigma}_{\mathrm{CF}}$. The response $K$ can then be obtained by using Eqns. 122, 121 and 64 .

\subsubsection{Separating Singular Fermi Liquid Coefficients}

As discussed above, one expects that the effective mass, which determines the energy scale of the low energy excitations, should be set by the Coulomb interaction scale. Similarly, one expects ${ }^{43}$ that the interaction function $f(\theta)$ should be on the interaction scale (i.e., proportional to $1 / \mathrm{m}^{*}$ ). However, as discussed above, we must satisfy the constraints Eqs. 91 and 89 on the values of $f_{1}$ and $f_{0}$ respectively. From Eq. 91 it is clear that $f_{1}$ is on the larger scale $1 / m_{\mathrm{b}}$ rather than the interaction scale. However, we now also claim that the constraint Eq. 89 fixes $f_{0}$ to be on the scale $1 / m_{\mathrm{b}}$ also. This counterintuitive result is due to the fact that the compressibility derivative $\frac{d \mu}{d n}$ is taken at fixed $\Delta B$. One can understand this ${ }^{43,44}$ by realizing that the Fermi liquid theory uses the mean field zero effective field solution for its ground state. When a particle is added or subtracted, in order to maintain a Fermi liquid (i.e., zero effective field), the external field must increased by $\tilde{\phi}$ flux quanta to compensate for the added Chern-Simons field. Thus, at fixed $\Delta B=0$, the magnetic field is linked to the density $n$ via $B=\tilde{\phi} n \phi_{0}$. In the limit $m_{\mathrm{b}} \rightarrow 0$, the interaction energy between the magnetization $\mathbf{M}=\mu_{\mathrm{b}} n$ and the external field is given by $E=\mathbf{M} \cdot \mathbf{B}=\pi \tilde{\phi} \hbar^{2} n^{2} / m_{\mathrm{b}}$. Of course this can also be thought of as the cyclotron energy. Differentiating this with respect to $n$ we obtain a magnetization contribution to the chemical potential

$$
\mu^{\mathrm{mag}}=\frac{2 \pi \tilde{\phi} \hbar^{2} n}{m_{\mathrm{b}}}=\hbar \omega_{\mathrm{c}}
$$

such that the magnetization contribution $\tilde{f}_{0}$ to the zeroth Fermi liquid coefficient $f_{0}$ is given by

$$
\tilde{f}_{0}=\frac{d \mu \mathrm{mag}}{d n}=\frac{2 \pi \tilde{\phi} \hbar^{2}}{m_{\mathrm{b}}}
$$

which is also the inverse compressibility of free electrons of mass $m_{\mathrm{b}}$ at constant $\Delta B$. The coefficient $f_{0}$ is written $f_{0}=\tilde{f}_{0}+\delta f_{0}$ where $\tilde{f}_{0}$ is $\mathcal{O}\left(m_{\mathrm{b}}^{-1}\right)$ and $\delta f_{0}$ is on the smaller interaction scale. As mentioned in Ref. 43 , in the limit $m_{\mathrm{b}} \rightarrow 0$, the requirement that the low energy spectrum is independent of $m_{\mathrm{b}}$ forces the other interaction coefficients $\left(f_{l}\right.$ for $\left.l \neq 0,1\right)$ to be on the interaction scale. In addition we note that using the Pauli exclusion principle a sum rule can be derived $^{18}$ for the remaining Fermi liquid coefficients $f_{l}$ for $l \neq 0,1$.

Since in the limit of $m_{\mathrm{b}} \rightarrow 0, \tilde{f}_{0}$ and $f_{1}$ are on the bare mass scale whereas all other coefficients $f_{l}$ (as well as $\delta f_{0}$ ) are expected to be on the smaller 
interaction scale, we will separate out the contributions of these two coefficients by writing

$$
\tilde{\Pi}^{-1}=\left[\tilde{\Pi}^{*}\right]^{-1}+\tilde{\mathcal{F}}_{0}+\mathcal{F}_{1}
$$

where

$$
\tilde{\mathcal{F}}_{0}=\left(\begin{array}{cc}
\tilde{f}_{0} & 0 \\
0 & 0
\end{array}\right) .
$$

is analogous to Eq. 99 and $\mathcal{F}_{1}$ is given by Eq. 106. As described in section 4.1.5 above, the function $\tilde{\Pi}^{*}$ is to be calculated using a Landau-Boltzmann equation representing quasiparticles with effective mass $m^{*}$ and interaction coefficients $f_{l}$ except that $f_{1}$ is artificially set to zero and the magnetic contribution $\tilde{f}_{0}$ is subtracted off of $f_{0}$. Once again, the form of Eq. 125 looks like the form of Eq. 64 where we have separated two interaction terms and defined the remaining response $\tilde{\Pi}^{*}$ to be the response of a similar Fermi liquid with those interactions removed.

The separation of the coefficients $\tilde{f}_{0}$ and $\tilde{f}_{1}$ are just the prescriptions given in Eq. 98 and 100 respectively. Having made this separation, we expect that the response $\tilde{\Pi}^{*}(q, \omega)$ is independent of $m_{\mathrm{b}}$ in the limit $m_{\mathrm{b}} \rightarrow 0$ and is well behaved for all values of $q / m_{\mathrm{b}}$. The transformation Eqns. 64, 105, 106, 121, and 125 do not in themselves involve any approximations, and may be considered simply as a means of defining a new 'irreducible' response function $\tilde{\Pi}^{*}(q, \omega)$.

\subsubsection{Relation to $M^{2} R P A$}

To relate this Fermi liquid approach to the $\mathrm{M}^{2} \mathrm{RPA}$ we note the identity $C+\tilde{\mathcal{F}}_{0}=M^{\dagger-1} C M^{-1}$ which holds in the limit $m_{\mathrm{b}} \rightarrow 0$. This identity is a statement of the fact that if you allow the magnetization to see the ChernSimons magnetic field as well as the external magnetic field, then the $1 / m_{\mathrm{b}}$ contribution to $f_{0}$ will vanish since the magnetization now sees zero magnetic field on average. We will also need $V=M^{\dagger} V M$ and $\mathcal{F}_{0}=M^{\dagger} \tilde{\mathcal{F}}_{0} M$ which is just the statement that a density-density interaction does not care whether or not the particles are magnetized. Using these identities, we find that $\mathrm{M}^{2} \mathrm{RPA}$ defined in Eq. 118 is equivalent to approximating $\Pi^{*}$ by $K^{0 *}$, the response of a free Fermi gas of particles of mass $m^{*}$, and calculating the response using Eqns. 64, 121, and 125.

We note that in Fermi liquid theory, the Landau-Boltzmann equation does not correctly describe the Landau diamagnetic contribution to the transverse static response. Similarly, we suspect that here the function $\tilde{\Pi}_{11}^{*}$ derived from the Landau-Boltzmann equation lacks a term of the form $q^{2} \chi$ where $\chi$ is some appropriate Landau susceptibility which we expect to be on the scale of the interaction strength. As usual, if we fix the ratio $\omega / q$ to be nonzero, and 
take $q \rightarrow 0$, this diamagnetic term becomes negligible. However, when $\tilde{\Pi}^{*}$ is approximated as $K^{0 *}$ for the $\mathrm{M}^{2} \mathrm{RPA}$, this diamagnetic contribution is included at least approximately.

To summarize this section, we have found that the magnetization contributions to the response can be properly obtained by declaring the Fermi liquid quasiparticle to be a magnetized object. This difference in the magnetization of the quasiparticle compared to the magnetization of the bare particle is similar to the renormalization of the particle mass that typically occurs in a Fermi liquid theory, but here is uniquely a result of the Lowest Landau level properties of the quantum Hall system.

\section{Perturbative Approaches and Trouble in the Infrared}

The MRPA and $\mathrm{M}^{2} \mathrm{RPA}$ ) discussed above are semi-phenomenological approximations. In this section, we will consider a more systematic perturbative approach to the Chern-Simons problem. We will see in section 6.1 that the proper expansion parameter is essentially $\tilde{\phi}$, the number of flux quanta attached to each fermion. Admittedly, $\tilde{\phi}$ is not small in the physical case. However, considering it to be a small parameter allows us to organize a perturbation expansion. In section 6.2 we will write down the diagrammatic rules for such a perturbative expansion and we will re-derive the RPA as well as obtaining the RPA screened gauge field propagator and the resulting fermion self-energy at lowest order. We will find in section 6.3 that the self-energy at $\nu=\frac{1}{2 m}$ is singular due to infrared gauge field fluctuations. We discuss the effect of this divergence on the effective mass (which will turn out to diverge) and on the physical response (which remains well behaved). Finally, in section 6.4 we try to see how the results obtained in this section are consistent with the Fermi liquid picture discussed above in the proceeding to sections. The main realization of this section is that although the single quasiparticle properties are singular, smooth excitations of the Fermi surface (which are those involved in transport at small $q$ and $\omega$ ) are well behaved so that we can in some cases work with a finite effective mass.

Throughout this section, for simplicity, we will focus on the $\nu=\frac{1}{2 m}$ Fermi liquid like state such that there is no residual magnetic field. We will also use a Lagrangian approach to analyze the problem. Aspects of such field theoretical formalisms were developed by a number of authors in a number of contexts including the bosonic picture of the fractional quantized Hall effect ${ }^{9}$ and anyon superconductivity ${ }^{10^{-12}}$. This field theoretical formalism was first used to describe composite fermions by Lopez and Fradkin who focused on the fractional quantized Hall states ${ }^{13}$. (The interested reader is encouraged to read 
their chapter in this book.) A brief discussion of the Lagrangian approach is also given by Halperin, Lee, and Read ${ }^{14}$ in the context of the even denominator states.

The action describing the Chern-Simons Hamiltonian (Eq. 12) at $\nu=\frac{1}{2 m}$ is given by (In this section we will often set $\hbar=e=c=1$ for convenience, returning these factors sporadically, when they are helpful to our understanding)

$$
\begin{aligned}
& S=\int d \mathbf{r} \int d t\left\{\psi^{*}\left(i \partial_{t}-a_{0}\right) \psi+\frac{1}{2 m_{\mathrm{b}}}\left|\left(i \nabla+\frac{e}{c} \mathbf{a}-\frac{e}{c} \mathbf{A}\right) \psi\right|^{2}\right. \\
& \left.+\frac{1}{\tilde{\phi} \phi_{0}} a_{0}(\nabla \times \mathbf{a})+\frac{1}{\tilde{\phi}^{2} \phi_{0}^{2}} \int d \mathbf{r}^{\prime}[\nabla \times \mathbf{a}(\mathbf{r})] v\left(\mathbf{r}-\mathbf{r}^{\prime}\right)\left[\nabla \times \mathbf{a}\left(\mathbf{r}^{\prime}\right)\right]\right\}
\end{aligned}
$$

Here, the explicit dependence of the fields on $\mathbf{r}$ and $t$ is omitted wherever there is no risk of confusion, and $\partial_{t} \equiv \partial / \partial t$. The first two terms in the action are just the action for a free fermion coupled to a gauge field. The $a_{0}(\nabla \times \mathbf{a})$ term is included to attach the Chern-Simons flux to the fermions. This can be seen by integrating out $a_{0}$ explicitly (which is trivial since the action is linear in $a_{0}$ ) to yield the usual constraint $\left(\tilde{\phi} \phi_{0}\right) n(\mathbf{r})=\left(\tilde{\phi} \phi_{0}\right) \psi^{*} \psi=\nabla \times \mathbf{a}$. In other words, $a_{0}$ is just a Lagrange multiplier for this constraint. The last term in the action is just the Coulomb interaction between density at position $\mathbf{r}$ and density at position $\mathbf{r}^{\prime}$ rewritten using the constraint.

\subsection{The Question of a Small Parameter}

The usual way to proceed at this point is to treat the coupling between the fermions and the gauge field as weak and perform a perturbative diagrammatic expansion. But before making any calculations with this action, we should attempt to identify the putative small parameter of the theory. To this end, it is convenient to re-express the action in terms of dimensionless variables and follow an argument by Stern and Halperin ${ }^{43}$. We express length in units of the magnetic length by defining $\tilde{\mathbf{r}}=\mathbf{r} / l_{B}$, and time in units of the inverse cyclotron frequency by defining $\tilde{t}=\omega_{\mathrm{c}} t$. We then rescale the fields accordingly, by defining $\tilde{\psi}=l_{B} \psi, \tilde{\mathbf{a}}=l_{B} \mathbf{a} / \phi_{0}, \tilde{\mathbf{A}}=l_{B} \mathbf{A} / \phi_{0}$, and $\tilde{a_{0}}=a_{0} /\left(\hbar \omega_{\mathrm{c}}\right)$. Specializing to the case of the Coulomb interaction $v(r)=e^{2} /(\epsilon r)$, the dimensionless action then becomes,

$$
\begin{aligned}
\tilde{S} & =\int d \tilde{\mathbf{r}} \int d \tilde{t}\left[\tilde{\psi}^{*}\left(i \partial_{\tilde{t}}-\tilde{a}_{0}\right) \tilde{\psi}+\frac{1}{2}|(i \tilde{\nabla}+\tilde{\mathbf{a}}-\tilde{\mathbf{A}}) \tilde{\psi}|^{2}+\right. \\
& \left.+\frac{1}{\tilde{\phi}} \tilde{a}_{0}(\tilde{\nabla} \times \tilde{a})+\frac{E_{\mathrm{c}}}{\hbar \omega_{\mathrm{c}}} \int d \tilde{\mathbf{r}}^{\prime}[\tilde{\nabla} \times \tilde{\mathbf{a}}(\tilde{\mathbf{r}})] \frac{1}{\left|\tilde{\mathbf{r}}-\tilde{\mathbf{r}}^{\prime}\right|}\left[\tilde{\nabla} \times \tilde{\mathbf{a}}\left(\mathbf{r}^{\prime}\right)\right]\right]
\end{aligned}
$$


where $E_{\mathrm{c}}=e^{2} /\left(\epsilon l_{B}\right)$ is the typical Coulomb energy, and where, again, the dependence of fields on $\tilde{\mathbf{r}}, \tilde{t}$ was omitted in most places, for brevity. As clearly seen from Eq. 128, the problem includes two dimensionless parameters. The first, $\tilde{\phi}$, is the number of flux quanta attached to each fermion. The second, $E_{\mathrm{c}} /\left(\hbar \omega_{\mathrm{c}}\right)$ is the ratio of the typical Coulomb energy to the cyclotron energy. In the action (Eq. 128), $\tilde{\phi}$ is the fermion-gauge field coupling constant, while $E_{\mathrm{c}} /\left(\hbar \omega_{\mathrm{c}}\right)$ is the parameter controlling the gauge field fluctuations. The relevant physical values of $\tilde{\phi}$ are $\tilde{\phi}=2,4,6 \ldots$, none of which are small. The physical value of $E_{\mathrm{c}} /\left(\hbar \omega_{\mathrm{c}}\right)$ can be assumed to be much smaller than one corresponding to the high magnetic field (or small $m_{\mathrm{b}}$ ) limit.

A perturbation expansion in which $\tilde{\phi}$ is held fixed at an even integer, while $E_{\mathrm{c}} /\left(\hbar \omega_{\mathrm{c}}\right)$ is turned on is singular due to the macroscopic degeneracy of the lowest Landau level. In the action (Eq. 128) this singularity is reflected by the fact that for even $\tilde{\phi}$, a small value of $E_{\mathrm{c}} /\left(\hbar \omega_{\mathrm{c}}\right)$ leads to strong gauge field fluctuations, making the mean field starting point invalid.

Thus, the perturbation expansion starts from $\tilde{\phi}=e^{2}=0$, i.e., from the problem of non-interacting electrons at zero magnetic field (which we know how to solve). The two parameters should then be turned on together ${ }^{a}$ such that the problem becomes that of weakly interacting anyons at weak magnetic field. When $\tilde{\phi}=2$ and $e$ gets to its physical value, the problem is that of interacting electrons at $\nu=1 / 2$.

While this procedure is probably a good start towards understanding this Chern-Simons system in the perturbative limit, it cannot solve the problem of the largeness of the physically relevant value of $\tilde{\phi}$. This problem is most clear in the limit $E_{\mathrm{c}} /\left(\hbar \omega_{\mathrm{c}}\right) \rightarrow 0$. In that limit, the physics of the system depends in a non trivial way on $\tilde{\phi}$. For even values of $\tilde{\phi}$, the action (Eq. 128) describes fermions in a strong magnetic field in a filling factor smaller than one, whereas for $\tilde{\phi}$ odd, the action describes bosons in a strong magnetic field. For any integer $\tilde{\phi}$, in the limit $E_{\mathrm{c}} /\left(\hbar \omega_{\mathrm{c}}\right) \rightarrow 0$, the fermion ground state and low energy excitations are confined to the lowest Landau level, and are therefore independent of the bare mass $m_{\mathrm{b}}$. In contrast, for non-integer values of $\tilde{\phi}$ the action (Eq. 128) describes anyons in a magnetic field. Since anyon's wavefunctions are non-analytic, they are not confined to the lowest Landau level and their low energy excitations are not independent of the bare mass. A perturbation expansion in $\tilde{\phi}$ is likely to miss, at least partially, this aspect.

Many - if not most - analytic calculations performed in the field of composite fermion physics are in some sense perturbations in small $\tilde{\phi}$ as described above. RPA, for example is lowest order perturbation theory made self consistent. A very closely related expansion ${ }^{45,44}$ is to introduce $N$ species of fermions

\footnotetext{
${ }^{a}$ The actual procedure for turning these on is discussed in Ref. 43.
} 
and expand in $1 / N$ (of course, at the end of the day, we must set $1 / N=1$ which is not small, but nonetheless this gives us a slightly different avenue for analyzing the problem). Several alternative approaches have also been attempted and should be briefly mentioned. Renormalization group calculations ${ }^{47}$ and Bosonization $^{48}$ have been used to nonperturbatively describe the low energy properties of the system. The Eikonal approximation is another nonperturbative approach that has been attempted ${ }^{46}$. Finally, we mention that several recent approaches attempting to re-describe the composite fermion system as a system of neutral dipoles ${ }^{49-53}$ will be described in depth in section 7 below. Although many of these approaches differ drasticly with each other, they seem to be converging on a single picture of the composite fermion Fermi liquid. In this section we will focus on the perturbation theory in small $\tilde{\phi}$, which is by far the most studied direction. At the end of the day, however, we will want to imagine turning up $\tilde{\phi}$ to its physical value.

\subsection{Diagrammatics}

We now return back to the action (Eq. 127) and consider a perturbative expansion as discussed above. Thus we need to figure out what sort of diagrams we can draw. To this end, we rewrite the action in a convenient form

$$
S=\int d t\left(\mathcal{L}_{\mathrm{f}}+\mathcal{L}_{\mathrm{af}}+\mathcal{L}_{\mathrm{a}}\right)
$$

with $\mathcal{L}_{\mathrm{f}}$ the the Lagrangian for free noninteracting fermion, $\mathcal{L}_{\mathrm{af}}$ the coupling between the fermions and the gauge field, and $\mathcal{L}_{\mathrm{a}}$ the bare gauge field Lagrangian (In Eq. 127, the first line is $\mathcal{L}_{\mathrm{f}}+\mathcal{L}_{\text {af }}$ and the second line is $\mathcal{L}_{\mathrm{a}}$ ). It is now convenient to rewrite the bare gauge field Lagrangian as

$$
\mathcal{L}_{\mathrm{a}}=\int d \mathbf{r} \delta a_{\mu}\left[\left(\mathcal{D}^{0}\right)^{-1}\right]_{\mu \nu} \delta a_{\nu}
$$

where, $\left[\mathcal{D}^{0}\right]^{-1}$ is the Fourier transform of

$$
\left(\mathcal{D}^{0}\right)^{-1}=\left(\begin{array}{cc}
0 & \frac{i q}{2 \pi \tilde{\phi}} \\
\frac{-i q}{2 \pi \tilde{\phi}} & \frac{v(q) q^{2}}{(2 \pi \tilde{\phi})^{2}}
\end{array}\right)
$$

which is known as the bare gauge field propagator. Here, we have defined

$$
\delta a_{\mu}=a_{\mu}-A_{\mu}
$$

for simplicity of notation so that the mean field situation is now $\delta a_{\mu}=0$, and we have used Coulomb gauge $\nabla \cdot \delta \mathbf{a}=0$ and $2 \times 2$ matrix notation. We note 
that generally, a gauge field propagator is defined as

$$
\mathcal{D}_{\mu \nu}(\mathbf{r}, t)=\left\langle T \delta a_{\mu}(\mathbf{r}, t) \delta a_{\nu}(0,0)\right\rangle
$$

with $T$ the time ordering operator. Here, $\mathcal{D}^{0}$ is this correlator for the free gauge field. We note that the bare gauge field propagator is nothing but the interaction matrices from section 3 above

$$
\mathcal{D}^{0}=-(C+V)
$$

which further justifies our earlier derivation of these interaction matrices. Diagrammatically, we will draw $\mathcal{D}^{0}$ as a dotted line

$$
\mathcal{D}_{\mu \nu}^{0}=\mu \text { - }-1-1-1-\cdot-\cdot \nu
$$

We also have a bare (free) propagator for the fermions (from $\mathcal{L}_{\mathrm{f}}$ ) which we write in the usual way ${ }^{31}$ as

$$
G_{\mathrm{f}}^{0}(\mathbf{k}, \omega)=\frac{1}{\hbar \omega-\xi_{\mathbf{k}}+i 0^{+} \operatorname{sgn}(\omega)}
$$

with $0^{+}$a positive infinitesimal, and

$$
\xi_{k}=\frac{\hbar^{2} k^{2}}{2 m_{\mathrm{b}}}-\mu \approx v_{\mathrm{F}}\left(k-k_{\mathrm{F}}\right)
$$

with $\mu$ the chemical potential and $v_{\mathrm{F}}=p_{\mathrm{F}} / m_{\mathrm{b}}$ the bare Fermi velocity. (The generalization of $G_{\mathrm{f}}^{0}$ to describe free fermions in finite magnetic field should be straightforward $\left.{ }^{31}\right)$. We draw this fermion propagator as a solid line

$$
G_{\mathrm{f}}^{0}=\longrightarrow(\mathbf{k}, \omega)
$$

To write the vertices, we now look to the interaction piece of the Lagrangian

$$
\mathcal{L}_{\mathrm{af}}=\int d \mathbf{r}\left(-\delta a_{0}(n-\bar{n})-\mathbf{J} \cdot \delta \mathbf{a}+\frac{n}{2 m_{\mathrm{b}}}|\delta \mathbf{a}|^{2}\right)
$$

with $n=\psi^{*} \psi$ the density, and $\mathbf{J}=\frac{1}{2 m_{\mathrm{b}}}\left[\psi^{*}(-i \nabla) \psi+\right.$ h.c. $]$ the paramagnetic part of the current (the physical current being given here by $\mathbf{J}-\frac{n}{m_{\mathrm{b}}} \delta \mathbf{a}$ ). The first two terms give interaction vertices which we write as

$$
\begin{aligned}
v_{0}\left(\mathbf{k}, \mathbf{k}^{\prime}\right) & =1 \\
v_{1}\left(\mathbf{k}, \mathbf{k}^{\prime}\right) & =\mathbf{J} \times \frac{\mathbf{k}-\mathbf{k}^{\prime}}{\left|\mathbf{k}-\mathbf{k}^{\prime}\right|}=\frac{1}{m_{\mathrm{b}}} \frac{\mathbf{k} \times \mathbf{k}^{\prime}}{\left|\mathbf{k}-\mathbf{k}^{\prime}\right|}
\end{aligned}
$$


which is written diagrammatically as

$$
v_{\mu}\left(\mathbf{k}, \mathbf{k}^{\prime}\right)=\quad \mathbf{k} \longrightarrow \mathbf{k}^{\prime}
$$

Finally, the last term in Eq. 137 gives the interaction vertex

$$
w_{\mu \nu}=\frac{1}{m_{\mathrm{b}}} \delta_{\mu 1} \delta_{\nu 1}=\because{ }^{\prime}{ }^{\prime}, \therefore \nu
$$

We now can go ahead and start writing diagrams. Perhaps the first thing we should do is to recover the response $K^{0}$ of noninteracting fermions coupled to an external gauge field. Without using the gauge propagator (i.e., for noninteracting fermions), we can draw two diagrams ${ }^{b}, D^{0}$, the current correlator, and $E$ the diamagnetic term. The current-current correlator is given diagrammatically as

$$
\begin{gathered}
D_{\mu \nu}^{0}(\mathbf{q}, \omega)=\int \frac{d \mathbf{k}}{(2 \pi)^{2}} \int \frac{d \Omega}{2 \pi} v_{\mu}(\mathbf{k}, \mathbf{q}-\mathbf{k}) v_{\nu}(\mathbf{q}-\mathbf{k}, \mathbf{k}) G_{\mathrm{f}}^{0}(\mathbf{k}, \Omega) G_{\mathrm{f}}^{0}(\mathbf{q}-\mathbf{k}, \omega-\Omega) \\
=
\end{gathered}
$$

(Here and below we use the usual diagrammatic rules ${ }^{31}$ to write the diagrams as integrals). This is really just the Fourier transform of the correlator

$$
D_{\mu \nu}^{0}\left(\mathbf{r}, t ; \mathbf{r}^{\prime}, t^{\prime}\right)=\left\langle T j_{\mu}(\mathbf{r}, t) j_{\nu}\left(\mathbf{r}^{\prime}, t^{\prime}\right)\right\rangle
$$

with $T$ the time-ordering operator and where here $j_{0}$ is the density operator and $j_{1}$ is the transverse paramagnetic current operator.

The diamagnetic term, on the other hand, is given by

$$
E_{\mu \nu}=\bigcirc=w_{\mu \nu} \int \frac{d \mathbf{k}}{(2 \pi)^{2}} \int \frac{d \omega}{2 \pi} G_{\mathrm{f}}^{0}(\mathbf{k}, \omega)=\frac{n_{\mathrm{e}}}{m_{\mathrm{b}}} \delta_{\mu 1} \delta_{\nu 1}
$$

Since these are the only things we can draw without the gauge propagator, we have the noninteracting response function given by

$$
K^{0}=D^{0}+E
$$

or

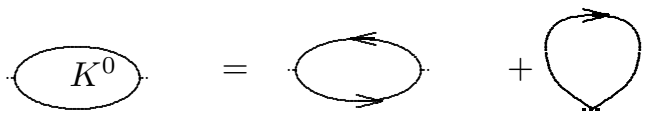

${ }^{b}$ With great apologies, I must warn the reader not to confuse $D$, the current-current correlator for $\mathcal{D}$ the gauge field propagator. Often they are both written as $D$. 
We can now consider the propagation of the gauge field to construct the RPA series

$$
K^{\mathrm{RPA}}=K^{0}+K^{0} \mathcal{D}^{0} K^{0}+K^{0} \mathcal{D}^{0} K^{0} \mathcal{D}^{0} K^{0}+\ldots
$$

which is precisely the same as the RPA prescription discussed above in section 3 . We write this diagrammatically as

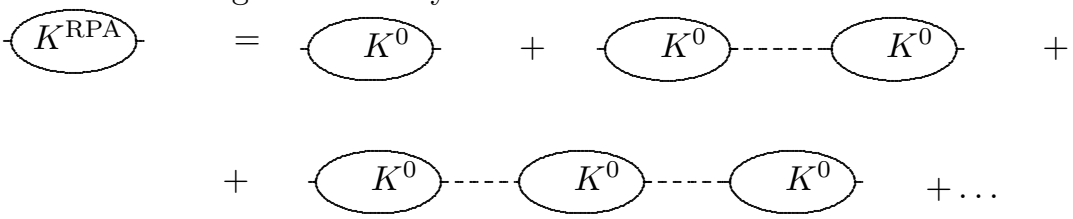

One other thing we might consider is the RPA screened gauge propagator $\mathcal{D}^{\mathrm{RPA}}$ which we draw diagrammatically as a boldface dotted line. We have

$$
\begin{aligned}
\mathcal{D}^{\mathrm{RPA}} & =\mathcal{D}^{0}+\mathcal{D}^{0} K^{0} \mathcal{D}^{0}+\mathcal{D}^{0} K^{0} \mathcal{D}^{0} K^{0} \mathcal{D}^{0}+\ldots \\
& =\mathcal{D}^{0}+\mathcal{D}^{0} K^{\mathrm{RPA}} \mathcal{D}^{0}
\end{aligned}
$$

or

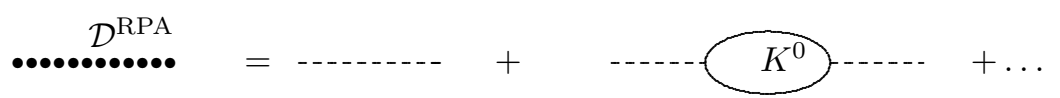

To gain intuition for the meaning of this screened propagator, we write an effective Lagrangian for the pieces $\mathcal{L}_{f}+\mathcal{L}_{\text {af }}$

$$
\mathcal{L}_{\mathrm{f}+\mathrm{af}}^{\mathrm{eff}}=-e \int d \omega \int d \mathbf{q} \delta a_{\mu}(\omega, \mathbf{q}) K_{\mu \nu}^{0} \delta a_{\nu}(-\omega,-\mathbf{q})
$$

In field theorist language, we have integrated out the fermions at 1-loop order (the 1 loop being the above diagrams of $D^{0}$ and $E$ ). To see that this effective Lagrangian is correct, all we need to do is recall that the physical current should be given by $j_{\mu}=-\delta S / \delta a_{\mu}=e K_{\mu \nu}^{0} \delta a_{\nu}$.

To find the propagator $\mathcal{D}$ at 1-loop, or RPA level, we simply write an effective Lagrangian for the full action 129

$$
\mathcal{L}_{\mathrm{f}+\mathrm{af}+\mathrm{a}}^{\mathrm{eff}}=\int d \omega \int d \mathbf{q} \delta a_{\mu}(\omega, \mathbf{q})\left(\mathcal{D}^{-1}\right)_{\mu \nu} \delta a_{\nu}(-\omega,-\mathbf{q})
$$

Combining Eq. 147 and 130 we immediately obtain the RPA result (Eq. 145)

$$
\left[\mathcal{D}^{\mathrm{RPA}}\right]^{-1}=\left[\mathcal{D}^{0}\right]^{-1}-K^{0}
$$

We now examine some of the features of the gauge field propagator $\mathcal{D}$. First of all, there is a direct relation between the exact value of $K_{00}$ and the 
exact value of $\mathcal{D}_{11}$ (which is formally defined as the correlator Eq. 133). This should be obvious since the constraint requires $\phi_{0} \tilde{\phi} n=2 \pi \tilde{\phi} n=\nabla \times \mathbf{a}=q a_{1}$ so (in shorthand notation)

$$
\mathcal{D}_{11}=\left\langle\delta a_{1} \delta a_{1}\right\rangle=\left[\frac{2 \pi \tilde{\phi}}{q}\right]^{2}\langle\rho \rho\rangle=\left[\frac{2 \pi \tilde{\phi}}{q}\right]^{2} K_{00}
$$

Thus, the low energy long wavelength form of $\mathcal{D}_{11}$ should have a pole at $\omega \sim i q^{3} v(q)$ as does $K_{00}$ (This pole does not go away in MRPA although the coefficients may change).

The other important feature of the gauge field propagator is that the high energy form of of $\mathcal{D}_{00}$ is given roughly by

$$
\mathcal{D}_{00} \approx \frac{(2 \pi \tilde{\phi})^{2}}{q^{2}} K_{11}=\frac{(2 \pi \tilde{\phi})^{2}}{q^{2}} \frac{n_{\mathrm{e}}}{m_{\mathrm{b}}} \frac{\omega^{2}}{\omega_{\mathrm{c}}^{2}-\left(\omega+i 0^{+}\right)^{2}} .
$$

This high energy form of $K_{11}$ is guaranteed by Kohn's theorem and the $f$-sum rule as discussed above in section 3.8.

The next step beyond RPA in standard perturbation theory is to calculate self-energy corrections. The self energy $\Sigma$ is defined in term of the exact oneparticle Green's function $G_{\mathrm{f}}(\mathbf{k}, \omega)$ by

$$
G_{\mathrm{f}}(\mathbf{k}, \omega)=\frac{1}{\hbar \omega-\xi_{\mathbf{k}}-\Sigma(k, \omega)}
$$

We will now use the conventional perturbative expansions ${ }^{31}$ to calculate the self energy $\Sigma$ of the fermion. We can write an infinite series of diagrams contributing to the self energy. However, for us, it will be a sufficient challenge to write down the first nontrivial term.

The simplest self energy we could draw is the following diagram

$$
\Sigma^{(1)}=\stackrel{\vdots}{\ddots}=\int \frac{d \mathbf{k}^{\prime}}{(2 \pi)^{2}} \int \frac{d \Omega}{2 \pi} w_{\mu \nu} \mathcal{D}_{\mu \nu}\left(\mathbf{k}^{\prime}, \Omega\right)
$$

which is a frequency and wavevector independent constant and can therefore be considered as just a renormalization of the chemical potential (and is therefore uninteresting). In the diagram we have drawn the bare gauge propagator, although we could equally well have used the RPA screened propagator, and we still would have found just a trivial constant.

A more nontrivial contribution to the self energy which occurs at the same order is given by the diagram 


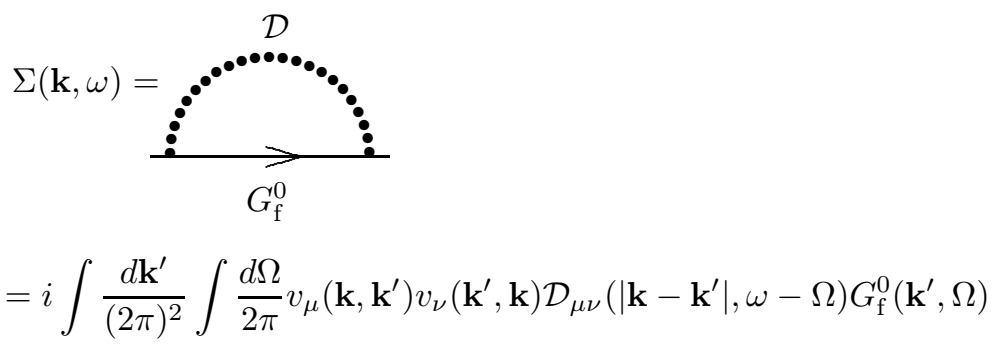

We can think of this as the process by which a free fermion of momentum $\mathbf{k}$ emits a gauge fluctuation of momentum $\mathbf{k}-\mathbf{k}^{\prime}$, both the fermion and the gauge field propagate, and then they interact (recombine) again at some later time. Note that by symmetry, the $\mathcal{D}_{01}$ and $\mathcal{D}_{10}$ terms vanish from the self energy and we need only consider $\mathcal{D}_{00}$ and $\mathcal{D}_{11}$.

In the very lowest order calculation that we could attempt, we could use the bare gauge field propagator $\mathcal{D}^{0}$ in Eq. 154 . However, we find that the $\mathcal{D}_{11}^{0}$ is zero and the only contribution is from the $\mathcal{D}_{00}^{0}=v(q)$ term which results in the usual self energy of free fermions interacting with themselves via a Coulomb interaction (We consider this contribution to be uninteresting).

At RPA level, we use the RPA value of the propagator $\mathcal{D}$ in Eq. 154 (as we have indicated in the diagram). At this level, the self energy Eq. 154 is both ultraviolet and infrared divergent.

We will begin by looking at the contribution $\delta \Sigma$ from the $\mathcal{D}_{00}$ term to the self energy Eq. 154. We obtain the result ${ }^{14}$

$$
\delta \Sigma(\mathbf{k}, \omega) \sim \ln \left(R q_{\max }\right)\left(\xi_{k}-\omega\right)+\ldots
$$

with $R$ the size of the system and $q_{\max }$ some ultraviolet cutoff. Since this term vanishes at $\omega=\xi_{k}$, it does not contribute to the effective mass of the fermion (see Eq. 162 below). The origin of this ultraviolet divergence is related to the energy required to instantaneously attach two flux quanta to a fermion. Turning on these two flux quanta suddenly has the effect of creating an electric field of strength $\tilde{\phi} / r$ a distance $r$ away. This field excites cyclotron oscillations at $\omega_{\mathrm{c}}$. Integrating $1 / r$ gives an energy cost for making this attachment that goes as

$$
E_{0} \sim \omega_{\mathrm{c}} \tilde{\phi} \ln \left(R q_{\max }\right)
$$

This issue is discussed in more detail by $\mathrm{HLR}^{14}$. A version of the ChernSimons theory that attaches the flux quanta adiabatically ${ }^{8}$ instead of suddenly, would presumably be able to stay completely in the lowest Landau level, and would not create these cyclotron excitations and would hence not have this singularity. Although such a lowest Landau level Chern-Simons theory has 
not been completely developed, recent work by Shankar and Murthy seems promising along this direction ${ }^{49}$.

\subsection{Infrared Divergences}

Most of the discussions of divergences in the Chern-Simons theory focus on the infrared problems, which are quite physical and have very interesting ramifications. These divergences come from the low energy overdamped mode of the transverse gauge field propagator $\mathcal{D}_{11}$. From here on, we will focus only on this term of the gauge field propagator since it is the only piece giving us these divergences (and hence this piece dominates).

We begin by plugging in the form of $\mathcal{D}_{11}$ given by Eqs. 150 and 68 into Eq. 154 to obtain the self energy ${ }^{43,14}$ (for the case of Coulomb interactions)

$$
\begin{aligned}
\Sigma(k, \omega) & =\frac{\tilde{\phi}^{2}}{2 \pi} \frac{\epsilon \hbar^{2} k_{\mathrm{F}}}{m_{\mathrm{b}} e^{2}} \hbar \omega \ln \left(\frac{4 e^{2} k_{\mathrm{F}}}{\tilde{\phi}^{2} \epsilon \omega}\right)+i \frac{\tilde{\phi}^{2}}{4} \frac{\epsilon \hbar^{2} k_{\mathrm{F}}}{m_{\mathrm{b}} e^{2}} \hbar \omega \\
& \sim \omega \ln (i \omega)
\end{aligned}
$$

A detailed description of this calculation is given in Ref. 43. This self energy is mostly frequency dependent, similar to the self energy arising from electronphonon interaction in metals, and in contrast to the self energy resulting from electron-electron interaction in metals (which is mostly $k$ dependent).

For the case of short-range interactions of the form $v(r) \sim r^{-\eta}$ with $1<$ $\eta \leq 2$, it is found that the self energy is even more singular. The reason for the increased singularity is that density fluctuations on long length scales are easy to make when there are not long range interactions. These long range (infrared) density fluctuations couple to the gauge field (i.e., create local Chern-Simons fields) which interacts strongly with the fermions. Performing the calculation (Eq. 154) we obtain

$$
\Sigma \sim(i \omega)^{\frac{2}{\eta+1}} .
$$

On the other hand, if one considers longer range interactions $\left(v(r) \sim r^{-\eta}\right.$ with $\eta<1$ ), the interactions suppress long range density fluctuations and there there is no singular part of the self energy (One must then consider all of the pieces of the self energy since no single divergent piece dominates). We note also that for quantized states away from $\nu=\frac{1}{2}$, the divergences are cut off by the effective cyclotron frequency and the perturbation theory becomes better defined.

Once we have this self-energy, the excitation modes $\tilde{\omega}(k)$ of the system are given by the poles of the energy denominator of the exact Green's function 
(Eq. 152). To find the modes, we set

$$
\hbar \tilde{\omega}(k)-v_{\mathrm{F}}\left(k-k_{\mathrm{F}}\right)-\Sigma(\tilde{\omega}(k), k)=0
$$

(where we have approximated $\xi_{k} \approx v_{\mathrm{F}}\left(k-k_{\mathrm{F}}\right)$ ). The effective mass $m^{*}$ is then defined through the effective Fermi velocity $\hbar k_{\mathrm{F}} / m^{*}$, which, in turn, is the group velocity $d \tilde{\omega} /\left.d k\right|_{k=k_{\mathrm{F}}}$ of the excitations $\tilde{\omega}(\mathbf{k})$. The effective mass is then given by

$$
\begin{aligned}
m^{*}(\omega) & =k_{\mathrm{F}}\left(\left.\frac{d \tilde{\omega}(k)}{d k}\right|_{k=k_{\mathrm{F}}}\right)^{-1}=m_{\mathrm{b}} \frac{1-\left.\frac{\partial \Sigma}{\partial \hbar \omega}\right|_{\omega=0}}{1+\left.\frac{\partial \Sigma}{\partial \xi_{k}}\right|_{k=k_{\mathrm{F}}}} \\
& \sim \frac{(\tilde{\phi} \hbar)^{2}}{2 \pi} \frac{\epsilon k_{\mathrm{F}}}{e^{2}}|\ln \omega|
\end{aligned}
$$

in the low frequency limit for the case of Coulomb interactions, and

$$
m^{*} \sim \omega^{-\frac{\eta-1}{\eta+1}}
$$

for in the low frequency limit for the case of short range interactions $\left(v(r) \sim r^{\eta}\right.$ with $1<\eta \leq 2$ ). (For interactions longer ranged than Coulomb there is no divergence of the effective mass.) It should be noted that whereas the self energy given above is only a first order approximation, it is argued by Stern and Halperin ${ }^{43}$ that this expression (Eq. 162) for the effective mass is in fact exact in the $\omega \rightarrow 0$ limit, at least for the Coulomb case.

The physical significance of this effective mass is most evident slightly away from $\nu=\frac{1}{2 m}$. Here, the fractional Hall gaps for the fractions $\nu=p /(2 m p+1)$ are given by ${ }^{43,14}$

$$
E_{g}(\nu)=\frac{\hbar e \Delta B}{m^{*}(\nu) c}
$$

where $m^{*}(\nu)$ is the effective mass (Eq. 162) calculated self consistently at frequency $\omega=E_{g}(\nu) / \hbar$. Thus, for filling fraction $p /(2 m p+1)$ for very large $p$, we should measure gaps that go as $E_{g}(p) \sim 1 /(p \ln p)$. For small $p$, on the other hand, the divergences are cut off so these pieces do not dominate. In principle, one could then perform a systematic perturbation expansion without experiencing any infrared problems. However, it would remain a problem to eliminate $m_{\mathrm{b}}$ from the low energy physics (See however the approach by Shankar and Murthy ${ }^{49}$ ).

The divergence of the effective mass at $\nu=\frac{1}{2 m}$ is also reflected in the singular behavior of the low energy specific heat ${ }^{14,56}$. For a normal Fermi liquid we have $C_{v} \sim T m^{*}$. For the Chern-Simons Fermi liquid with Coulomb 
interactions, the effective mass diverges as $\log \omega$ for excitations on the scale $\omega$. Thus, the specific heat is given by $C_{v} \sim T \log T$. For short range interactions ${ }^{c}$, we similarly obtain $C_{v} \sim T^{2 /(1+\eta)}$.

Another important quantity we can extract from the self energy is the quasiparticle-quasiparticle scattering time $\tau_{q p}$. Usually one uses the rule that $\tau_{q p}^{-1}=\operatorname{Im} \Sigma$. However, here, we should be a bit more careful. More accurately, it is the imaginary part of the complex excitation frequency $\tilde{\omega}$ that determines the lifetime of the excitations via $\tau_{q p}^{-1}=\operatorname{Im} \tilde{\omega}$. Defining $\omega=\operatorname{Re}(\tilde{\omega}(k))$ we find that the imaginary part of $\tilde{\omega}$ is given by

$$
\begin{array}{rlr}
\frac{1}{\tau_{q p}} \equiv \operatorname{Im} \tilde{\omega} & \sim \frac{\omega}{\ln \omega} \quad \text { Coulomb } \\
& \sim \omega \quad \text { Short Range }
\end{array}
$$

which is a somewhat greater lifetime than the usual expression $\operatorname{Im} \Sigma$ would lead one to believe. In another language, the quasi-particle lifetime is lengthened because $\tau_{q p}^{-1}=Z \operatorname{Im} \Sigma$ rather than $\operatorname{Im} \Sigma$ where $Z^{-1}=1-\frac{d \Sigma}{d \omega}$ is the so-called wavefunction renormalization. Thus, $\omega \tau_{q p} \sim \ln |\omega| \gg 1$ for the Coulomb case, and the quasi-particle remains well defined as we approach the Fermi surface. However, for short range interactions, $\omega \tau_{q p} \approx 1$ so that the quasiparticle is not well defined.

One might be concerned that the singular behavior of the self-energy would result in a divergent response function. However, the self energy, like the exact Green's function itself, is not a gauge invariant quantity. When one calculates the gauge invariant electromagnetic response, one finds that singular vertex corrections exactly cancel the singular self energy terms to give a finite nonsingular response ${ }^{44,54}$. For example, the sum of the following three divergent diagrams is convergent

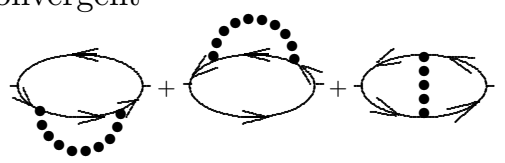

This nonsingular behavior of the physical response function is also obtained in several other approaches (including nonperturbative approaches) to analyzing the Chern-Simons problem ${ }^{45,48,46}$. Furthermore, it has been shown by Kim et $\mathrm{al}^{55}$ that one can write down a so-called quantum Boltzmann equation for the response of the Chern-Simons system that is nonsingular. In essence the realization is that although there is a singularity in the energy required to

\footnotetext{
${ }^{c}$ Note that for short-range interaction, we obtain an incorrect coefficient in front of the power law by using a quasiparticle picture due to the fact that the individual quasiparticle
} is not well defined. 
excite a single quasiparticle, smooth deformations of the Fermi surface (which are what are required for physical response functions at long wavelength and low frequency) are quite well behaved. In another language, one finds that even for short range interactions, the transport scattering time $\tau_{t r}$ (which enters the response functions) is given by ${ }^{44}$ (compare Eq. 166) $\tau_{t r}^{-1} \sim \omega^{4 /(1+\eta)}$ such that $\omega \tau_{t r} \ll 1$ yielding a nonsingular response.

\subsection{Divergent Fermi Liquid Theory}

Since excitation of a single quasiparticle is extremely singular in the case of short range interactions, we are prevented from writing down a conventional Landau Fermi liquid description for such a system (although a nonconventional Boltzmann-like description can be written down in the language of the quantum Boltzmann equation ${ }^{55}$ - even for the case of short range interactions). For the case of Coulomb interactions, however, we might hope that the divergences are not sufficiently singular to prevent us from describing the system in the conventional Landau Fermi liquid format. We would hope that the result of such a calculation would return us to the phenomenological Landau Fermi liquid approach discussed above in section 4 and 5.4. For the rest of this section, we focus on the Coulomb case.

As discussed above in section 4, Landau's Fermi liquid theory describes the system in terms of the parameters, $m^{*}$ and $f(\theta)$, which can be written formally in terms in terms of one- and two- particle Green's functions ${ }^{30}$. As discussed above, the effective mass is singular and must be written as a function of frequency. Similarly, perturbative calculation of the Landau quasiparticle interaction function $f(\theta)$ is singular. Although one might imagine regularizing this divergence in any one of a number of different ways, one very natural method was proposed in Ref. 43, where the interaction function $f(\theta)$ is written as a function of the energy exchange between the quasi-particles. (In conventional, non-singular, Fermi liquid theory, the Landau interaction involves zero energy exchange, since both quasi-particles lie on the Fermi surface). A natural lowest order calculation then yields the interaction function between quasiparticles (See Ref. 43 for details)

$$
f(\theta ; \omega)=\frac{2 \pi k_{\mathrm{F}}^{2}}{m_{\mathrm{b}} m^{*}(\omega)}\left(\cos ^{2} \frac{\theta}{2}\right) \mathcal{D}_{11}\left(2 k_{\mathrm{F}} \sin \frac{\theta}{2}, \omega\right)
$$

For zero frequency exchange, this function takes the singular form

$$
\lim _{\omega \rightarrow 0} f(\theta ; \omega)=\frac{(2 \pi \hbar)^{2}}{m_{\mathrm{b}}} \delta(\theta)
$$


The effect of such a singular interaction function is very simple, and is immediately understood when Eq. 168 is substituted into Eq. 94 to yield

$$
\delta \epsilon_{1}(\theta)=\frac{m^{*}}{m_{\mathrm{b}}} \nu(\theta) \gg \nu(\theta)
$$

Substituting into the Boltzmann transport equation (Eq. 93) in this approximation then yields

$$
-i \omega \nu(\theta)+\left(i q v_{\mathrm{F}} \cos (\theta)-\Delta \omega_{\mathrm{c}} \frac{\partial}{\partial \theta}\right) \nu(\theta)=-e \mathbf{E} \cdot \hat{\mathbf{n}}(\theta)+I(\theta)
$$

where $v_{\mathrm{F}}=p_{\mathrm{F}} / m_{\mathrm{b}}$ is the bare Fermi velocity, and $\Delta \omega_{\mathrm{c}}=e \Delta B / m_{\mathrm{b}} c$ is the bare fermion cyclotron frequency. The delta function $f(\theta)$ generates then a transport equation that is the same as that for a system where the effective mass is re-renormalized back to the band mass and the quasi-particles on the Fermi surface are non-interacting! Thus, if this approximation (Eq. 168) is correct, the long wavelength low frequency response of the system is that of non-interacting quasiparticles with an unrenormalized bare mass. Note that the magnitude of this delta function yields a value of $f_{1}$ set by the bare band mass in agreement with the constraint on $f_{1}$ given by Eq. 91 .

The expression (Eq. 168) for the Landau function $f(\theta ; \omega)$ is only an approximation valid to low order in $\tilde{\phi}$ as discussed above. We now consider ${ }^{39}$ what we might conclude about the exact Landau function for the physical value of $\tilde{\phi}$ an even integer, in the limit of $m_{\mathrm{b}} \rightarrow 0$. While $f_{0}$ and $f_{1}$ should depend on the bare mass in the limit $m_{\mathrm{b}} \rightarrow 0$ (See section 5.4.1 for the properties of $f_{0}$ ) this should not be the case for all other Landau parameters, since low $q, \omega$ linear response in that limit should be constrained to the lowest Landau level, i.e., independent of the bare mass ${ }^{43}$. Finally, as discussed above in section 6.3 , we expect the diverging effective mass $m^{*}$ to cancel from linear response functions of the $\nu=\frac{1}{2}$ state.

As demonstrated above, for the diverging $m^{*}$ to cancel from the linear response functions, the Landau function should include a $\delta$-function component. For the Landau parameters $f_{l}$ for $l \geq 2$ to be $m_{\mathrm{b}}$ independent, the amplitude of this $\delta$-function component should be independent of the bare mass, i.e., should be determined by the energy scale of electron-electron interaction $e^{2} /\left(l_{B} \epsilon\right)$. Thus by dimensional analysis, we define a new effective mass

$$
\frac{\hbar^{2}}{m^{* *}}=C(\nu) \frac{e^{2}}{l_{B} \epsilon}
$$

where $C(\nu)$ is an unknown dimensionless number of order 1 which is only very weakly dependent on $\nu$. It is this mass $m^{* *}$ that enters the phenomenological Landau-Boltzmann approaches (which we there call $m^{*}$ ) in sections 4 and 
5. Indeed, everywhere where a finite effective mass is used for calculating responses for the $\nu=\frac{1}{2 m}$ problem, it is this finite effective mass $m^{* *}$ and not the divergent effective mass that is being used. The critical thing to remember is that the effective mass $m^{* *}$ describes smooth deformations of the Fermi surface, not single quasiparticle excitations, and can therefore be nonsingular (whereas the true effective mass $m^{*}(\omega)$ describing single quasiparticle excitations is singular). The mass $m^{* *}$ yields a re-renormalized Fermi velocity $v_{\mathrm{F}}^{* *}=p_{\mathrm{F}} / m^{* *}$ which determines the edge of the low energy continuum of quasiparticle excitations. It is interesting to note that $v_{\mathrm{F}}^{* *}=C e^{2} /(\epsilon \hbar)$ is independent of density. We note that the effective mass $m^{* *}$, since it is non-divergent, is probably most closely related to the effective mass that determines the energy gaps of the fractional hall gaps well away from $\nu=\frac{1}{2 m}$ (fractions like $\frac{1}{3}$ and $\frac{2}{5}$ ). We note that estimates of this mass have been made by exact diagonalizations ${ }^{57,42,14}$.

Using this new mass scale, we propose the following conjecture for the interaction function $f(\theta)$ in the low frequency limit

$$
\lim _{\omega \rightarrow 0} f(\theta ; \omega)=(2 \pi \hbar)^{2}\left(\frac{1}{m^{* *}}-\frac{1}{m^{*}}\right) \delta(\theta)+f^{* *}(\theta)
$$

where $f^{* *}(\theta)$ is nonsingular in the low frequency limit. Here, the $\delta$ function term re-renormalizes the mass from the divergent $m^{*}(\omega)$ to the finite value $m^{* *}$ which is appropriate for calculating responses. The residual term $f^{* *}$ gives us the Landau interaction function for these effective particles of mass $m^{* *}$. Indeed, this is the Landau function we used in sections 4 and 5 above (which we just called $f$ there). We note that this Landau function must satisfy the constraints Eq. 91 and Eq. 89. These require that $m^{* *} f_{1}^{* *} /\left(2 \pi \hbar^{2}\right)=$ $\left(m^{* *} / m_{\mathrm{b}}\right)-1$ and that the leading term of $f_{0}^{* *}$ is given by $\pi \hbar^{2} \tilde{\phi} / m_{\mathrm{b}}$. All other Fourier modes $f_{l}^{* *}$ if $f^{* *}(\theta)$ should be independent of the bare mass.

To summarize this section, we have found that a perturbative approach to the Chern-Simons problem at $\nu=\frac{1}{2 m}$ forces us to suffer with infrared divergences of quantities such as the effective mass $m^{*}$. These divergences appear in the fractional Hall gaps and the specific heat near $\nu=\frac{1}{2}$, but do not appear in the response. For this reason, we believe that another finite effective mass (which here we call $m^{* *}$ ) can be defined that should enter into calculations of the response. This, in some sense, might justify our earlier use of a finite effective mass (which there we called $m^{*}$ ) in calculations in section 4 and 5 . However, more work remains to be done to determine how well justified this really is. One alternative possibility that one might consider is that the response at any given frequency $\omega$ must be calculated with a mass $m^{*}(\omega)$ appropriate for that frequency (which may diverge at small $\omega$ ). This would make the edge of the continuum of low energy quasiparticles quite curved. 
There is also the possibility that higher Fermi liquid coefficients conspire such that a great number of collective (yet discrete) zero sound modes merge into the continuum at low energies ${ }^{55}$.

At fractional Hall states $\frac{p}{2 m p+1}$ for small $p$, there is no divergence of $m^{*}$ and one could in principle calculate $m^{*}$ perturbatively. However, in a straightforward perturbative approach it is not clear how to get the bare mass to vanish from the answer as it should in the $m_{\mathrm{b}} \rightarrow 0$ limit (See however Ref. 49). Thus, we are still stuck with treating $m^{*}$ as a phenomenological parameter. Nonetheless, we would expect it to obey the scaling form of Eq. 171. Estimates of the coefficient $C(\nu)$ can be made by exact diagonalization ${ }^{42,57}$.

\section{Wavefunction Picture of Composite Fermions}

The success of the Chern-Simons approach should strike us as somewhat surprising. We have modeled an electron in a large magnetic field as a fermion bound to flux and all of a sudden we are getting many of the right answers at even a crude mean field level - and we get even more right answers when we consider corrections to mean field, such as RPA. To understand physically why this approach makes sense, it is useful to think in terms of wavefunctions. Although the analysis of wavefunctions is somewhat off of the main line of development of this paper, it will be useful for developing intuition for the physics. Those who desire a more thorough discussion of the wavefunction properties of composite fermions are referred to the works of Jain ${ }^{5,6}$ and $\operatorname{Read}^{58}$.

In the tradition begun by Laughlin ${ }^{23}$, we will attempt to guess trial wavefunctions for the states in which we are interested, and study the properties of these wavefunctions. Although such an a approach is far from systematic, it has greatly deepened our understanding of the fractional quantum Hall effect. Indeed, it is probably not an overstatement to say that Laughlin's wavefunction $^{23}$ is the single most important theoretical step ever made in the theory of fractional quantum Hall effect; and similarly, it should be recalled that the notion of the composite fermion was begun by $\mathrm{Jain}^{5}$ in the wavefunction language.

The experienced reader is encouraged to skip quickly through section 7.1 and 7.2, which should be review for anyone well versed in quantum Hall physics. Those in need of further introductory details regarding the wavefunction approaches are referred to Refs. 1-4. In section 7.3 we discuss the properties of the Rezayi-Read $\nu=\frac{1}{2}$ wavefunction and explain how it leads us to a picture of the composite fermion at $\nu=\frac{1}{2}$ as a neutral dipole. In section 7.4 we consider the existence of the cyclotron scale $R_{\mathrm{c}}^{*}$ in this wavefunction picture 
and show that it agrees with the Chern-Simons picture of HLR. In 7.5 we discuss the Jain wavefunctions for the fractionally quantized states and in section 7.6 we compare the wavefunction approaches to the Chern-Simons transforms discussed above. In section 7.7 we perform explicit calculations within this neutral dipole picture (with no Chern-Simons field in sight) and show that the results agree with the Chern-Simons approach discussed above.

\subsection{Wavefunctions and Lowest Landau Level Physics}

In order to study trial wavefunctions, we work in circular gauge, $\mathbf{A}=\frac{1}{2} \mathbf{B} \times \mathbf{r}$ and write the position of the $j^{t h}$ particle in its complex representation as ${ }^{62}$ $z_{j}=x_{j}+i y_{j}$. In this language, we can write an arbitrary many-electron wavefunction as

$$
\Psi\left(z_{1}, z_{2}, \ldots, z_{N}\right)=f\left(z_{1}, z_{2}, \ldots, z_{N}\right) \prod_{j=1}^{N} e^{-\frac{1}{4}\left|z_{j}\right|^{2} / l_{B}^{2}}
$$

The wavefunction $\Psi$ represents electrons restricted to the lowest Landau level (appropriate for the large $B$ or $m_{\mathrm{b}} \rightarrow 0$ limit) if and only if the function $f$ is analytic in all of its arguments (i.e., a polynomial containing only nonnegative powers of the $z$ 's and no powers of the $\bar{z}$ 's). We also point out that the wavefunction must be antisymmetric under interchange of the position of any two of the particles in order to be properly fermionic.

To analyze a lowest Landau level wavefunction we consider fixing the positions $^{59}$ of all but one of the electrons (say electrons 1 though $N-1$ ) and move the one remaining test electron (say electron $N$ ) adiabatically. With the positions $z_{1}, \ldots, z_{N-1}$ thus fixed, the wavefunction is now an analytic function of the position $z_{N}$ of this last electron times an exponential factor. At certain values of $z_{N}$ the value of the wavefunction will be zero. Because of the analyticity condition, as we move $z_{N}$ in a circle around any such zero, the wavefunction undergoes a phase rotation of $2 \pi$. For this reason the zeros of the wavefunction are also referred to as "vortices." We point out that since the wavefunction is antisymmetric (i.e., fermionic), it must go to zero when $z_{N}$ approaches the position of any other electron. Thus, there is a naturally at least one vortex at the position of each electron.

If we now move our test electron adiabatically in a large loop of area $\mathcal{A}$, the phase accumulated by the electron (the traditional Aharonov-Bohm phase) should be given by

$$
e^{i \frac{2 \pi}{\phi_{0}} \oint \mathbf{A} \cdot d l}=e^{i 2 \pi \Phi / \phi_{0}}
$$

where $\Phi=\mathcal{A} B$ is the flux enclosed. However, as mentioned above, the analyticity of the wavefunction also demands that the accumulated phase is given 
by $\exp 2 \pi i N_{z}$ where $N_{z}$ is the number of zeros of the wavefunction enclosed in the loop. Comparing these two results, we find that any lowest Landau level wavefunction must have $B / \phi_{0}$ zeros per unit area.

To create a density fluctuation in an arbitrary ground state wavefunction of uniform density, we can introduce a vortex at position $z_{0}$ by multiplying the wavefunction by the factor

$$
\prod_{j=1}^{N}\left(z_{j}-z_{0}\right) .
$$

Clearly, this factor suppresses the amplitude of the wavefunction in the vicinity of $z_{0}$ leaving a deficit of electrons (or positive charge) near $z_{0}$. We note that the multiplication of a wavefunction by this vortex operator increases the degree of the polynomial part of the wavefunction $(f)$, and thus increases the size of the droplet. This is just another way of saying that the density removed from near the point $z_{0}$ is accounted for by an increase in density at the edge of the system. In Laughlin's original work ${ }^{23}$, a plasma analogy was used to show that this vortex operator acting on a ground state wavefunction of the $\nu=\frac{1}{2 m+1}$ quantum Hall state (with $m$ an integer) results in a quasihole of charge $+\nu$. We will see next that this is a rather general result.

To evaluate the charge on the vortex, we consider adiabatically ${ }^{a}$ moving its position $z_{0}$ in a large loop of area $\mathcal{A}$. Analogous to the discussion above, the Aharonov-Bohm phase accumulated by this process is given by $\exp \left(2 \pi i q \Phi / \phi_{0}\right)$ where here $q$ is the charge on the vortex, and once again, $\Phi=B \mathcal{A}$ is the flux inclosed. On the other hand, from the form of Eq. 175 it is clear that the wavefunction picks up a phase of $\exp \left(2 \pi i N_{\mathrm{e}}\right)$ where $N_{\mathrm{e}}$ is the number of electrons enclosed in the loop. Thus, we conclude that the charge on the vortex is $q=N_{\mathrm{e}} \phi_{0} / B \mathcal{A}=\nu$.

As mentioned above, the number of zeros of the wavefunction with respect to the position of a test electron determines the applied magnetic field. In terms of counting these zeros, the introduction of the vortex (application of the operator Eq. 175 to a ground state wavefunction) corresponds to the increase of the applied magnetic field by a single flux quantum ${ }^{b}$. Indeed, one way to create such a density fluctuation is to adiabatically insert a single thin flux

\footnotetext{
${ }^{a}$ For compressible state there are low energy excitations so one cannot have adiabatic motion. Similarly, the argument given below in the next paragraph fails for a compressible state that is not a perfect Hall conductor. Although this issue has not been completely sorted out, one possible way to think about such compressible states is to consider a limit of a series of incompressible states with smaller and smaller gaps.

${ }^{b}$ This is analogous to a real hole being created when a single flux quantum is added to a filled Landau level (the $\nu=1$ case). Here, the increase in field by one flux quantum increases the degeneracy of the lowest Landau level by one, creating exactly one hole.
} 
quantum through the system at position $z_{0}$. As this flux quantum is turned on, an EMF is created in the direction going around the flux by Faraday's law. For a system that is a perfect Hall conductor, this moves charge away from the point $z_{0}$ (perpendicular to the EMF). To calculate the charge moved away from $z_{0}$ we write

$$
\frac{1}{c} \frac{d \Phi}{d t}=\oint \mathbf{E} \cdot d \mathbf{l}=\oint \rho \cdot \mathbf{j} \cdot d \mathbf{l}
$$

where $\Phi$ is the flux being turned on, $\rho$ is the resistivity matrix, and the path of integration goes around the point $z_{0}$. For quantized Hall states we can use the fact that the diagonal resistivity is zero, and this EMF can be integrated with respect to time to get

$$
\frac{1}{c} \Delta \Phi=\rho_{x y} \oint d \mathbf{l} \cdot \int_{t_{i}}^{t_{\mathrm{f}}} d t(\mathbf{j} \times \hat{z})=\rho_{x y} q
$$

where $q$ is the total amount of charge that is moved away from the point $z_{0}$ (i.e., the charge of the created vortex). Choosing $\Delta \Phi$ to be a single flux quantum, and recalling that $\rho_{x y}=h /\left(e^{2} \nu\right)$, then yields the correct vortex charge of $q=+\nu$.

\subsection{Laughlin's Wavefunction}

Now that we have discussed some of the properties of wavefunctions in the lowest Landau levels, we turn to writing down trial wavefunctions for fractional quantized Hall states. Such trial wavefunction guesswork might seem at first like trying to find the proverbial needle (the ground state wavefunction) in the haystack (the huge Hilbert space of possible wavefunctions). However, such guesswork has been extremely successful, and has been justified in retrospect by comparison to the results of exact diagonalizations of small systems $5,6,26,23,28$.

Laughlin's trial wavefunction ${ }^{23}$ for quantized Hall states at filling fractions $\nu=\frac{1}{2 m+1}$ is given by the expression

$$
\Psi_{\frac{1}{2 m+1}}\left(z_{1}, z_{2}, \ldots, z_{N}\right)=\prod_{i<j}\left(z_{i}-z_{j}\right)^{(2 m+1)} \prod_{i=1}^{N} e^{-\frac{1}{4}\left|z_{i}\right|^{2} / l_{B}^{2}}
$$

which, for $m$ an integer, is a properly antisymmetric (fermionic) wavefunction, and has the proper analytic times exponential (lowest Landau level) form. This wavefunction has $(N-1)(2 m+1)$ zeros with respect to the position of the $N^{t h}$ electron. Since there must be $B / \phi_{0}$ such zeros per unit area we immediately calculate that the filling fraction for this Laughlin state is indeed 
$\nu=n_{\mathrm{e}} \phi_{0} / B=\frac{1}{2 m+1}$. This Laughlin form has been shown to be the exact ground state wavefunction for a system with a certain type of short range interaction ${ }^{60,26}$. It has also been shown to have an extremely high overlap with the exact ground state wavefunction (calculated numerically) for small systems with Coulomb interactions ${ }^{23,26,28}$. It should also be noted that for $m=0$, the Laughlin wavefunction is exactly the (single Slater determinant) wavefunction for one filled Landau level.

To understand why the Laughlin form is such a good guess for a trial wavefunction, we once again consider moving one test electron adiabatically while leaving the position of all the other electrons fixed. Here, we see that all of the zeros (vortices) are attached to the position of the other electrons. In fact, the Laughlin wavefunction has a $2 m+1^{\text {th }}$ order zero as any $z_{i}$ approaches any other $z_{j}$, meaning that the wavefunction amplitude is highly suppressed as any two electrons approach each other. The higher the order of the zero of the wavefunction, the more the electrons stay away from each other. Indeed, the reason that this Laughlin wavefunction has such a good energy is that the zeros are arranged such that the electrons stay as far away from each other as possible, thereby lowering the Coulomb energy. Moreover, for a fixed filling fraction, there is a fixed number of zeros of the wavefunction, and the Laughlin form arranges to put all of these zeros at the position of the other electrons such that they are efficiently used to keep electrons away from each other, leaving no zeros "wasted".

\subsection{The $\nu=\frac{1}{2}$ Wavefunction}

One would naively guess that a good wavefunction for electrons at filling fraction $\frac{1}{2 m}$ would be an analogous Laughlin like state given by Eq. 178 only with an exponent $2 m$ rather than $2 m+1$. However, such a wavefunction is symmetric rather than antisymmetric under particle interchange, and therefore represents a bosonic rather than a fermionic wavefunction ${ }^{c}$. What one wants to do to obtain a good fermionic $\nu=\frac{1}{2 m}$ wavefunction is then to alter this bosonic wavefunction slightly to make it antisymmetric while doing "minimal damage" to the good correlation structure of the Laughlin state ${ }^{58}$. To this end one considers multiplying this bosonic wavefunction by the determinant

$$
\operatorname{Det}\left[e^{i \mathbf{k}_{i} \cdot \mathbf{r}_{j}}\right]=\left|\begin{array}{ccc}
e^{i \mathbf{k}_{1} \cdot \mathbf{r}_{1}} & e^{i \mathbf{k}_{1} \cdot \mathbf{r}_{2}} & \cdots \\
e^{i \mathbf{k}_{2} \cdot \mathbf{r}_{1}} & \ddots & \\
\vdots &
\end{array}\right|
$$

\footnotetext{
${ }^{c}$ It has indeed been shown ${ }^{63}$ that such a wavefunction is extremely good for bosons at $\nu=\frac{1}{2}$.
} 
To have an antisymmetric wavefunction, all of the $k_{i}$ 's must be different, but to keep the energy minimal ${ }^{d}$, we will choose the smallest possible values for all the $k_{i}$ 's so that the determinant (Eq. 179) becomes just a filled Fermi sea, i.e., the wavefunction of free fermions in zero magnetic field. Once this filled Fermi sea is multiplied by the bosonic Laughlin state the end result is the wavefunction for the $\nu=\frac{1}{2 m}$ state, often called the Rezayi-Read ${ }^{61}$ wavefunction, given by

$$
\Psi_{\frac{1}{2 m}}=\mathcal{P}_{\mathrm{LLL}} \operatorname{Det}\left[e^{i \mathbf{k}_{i} \cdot \mathbf{r}_{j}}\right] \prod_{i<j}\left(z_{i}-z_{j}\right)^{2 m} \prod_{i} e^{-\frac{1}{4}\left|z_{i}\right|^{2} / l_{B}^{2}}
$$

(where $\mathbf{r}_{i}$ and $z_{i}$ represent the same coordinate in the vector and complex notations respectively). Here we have added the projection operator $\mathcal{P}_{\text {LLL }}$ out front to re-project this wavefunction back to the lowest Landau level since the fermionic determinant has some part in higher Landau levels. Note that Jain ${ }^{6}$ might prefer to think of this as a fermionic wavefunction that is "composite fermionized" by multiplying by the composite fermionization factor ${ }^{6}$ (i.e., the bosonic Laughlin wavefunction), which attaches $2 m$ vortices to each electron. It has been shown that this Rezayi-Read wavefunction has very good overlap with the exact ground state for small systems ${ }^{61,6}$.

We can compare this procedure of "minimally damaging" a bosonic wavefunction to obtain a fermionic wavefunction with the situation at zero magnetic field for noninteracting (or weakly interacting) particles. In this case the ground state bosonic wavefunction is just $\Psi\left(\mathbf{r}_{1}, \ldots, \mathbf{r}_{n}\right)=1$. To get the ground state fermionic wavefunction, we "minimally damage" this good ground state bosonic state by multiplying by the determinant factor (Eq. 179) which makes the wavefunction antisymmetric if all the $k_{i}$ 's are chosen different, and minimizes the energy if the the $k_{i}$ 's take the minimum possible value filling a Fermi sea.

It is interesting to consider the effects of the multiplication by the projected determinant. Rewriting $\mathbf{r}$ in its complex coordinates, we have $\mathbf{k} \cdot \mathbf{r}=\frac{1}{2}(k \bar{z}+\bar{k} z)$ where the overbar means complex conjugation and $k$ is the complex coordinate representation for the vector $\mathbf{k}$. Projection to the lowest Landau level can be achieved by ${ }^{62}$ replacing $\bar{z}$ by $2 l_{0}^{2} \frac{d}{d z}$ where all derivatives are normal ordered to the left and the derivatives do not act on the exponential factors

\footnotetext{
${ }^{d}$ For the composite fermion wavefunctions, there is an over-completeness which allows us to move the center of the Fermi sea to any value of $k$ and still have the exact same physical wavefunction. This so called $\mathbf{K}$-invariance, along with the reason why we choose to keep the $k_{i}$ 's minimal to minimize the energy, will be discussed further below.
} 
$\exp \left(-\frac{1}{4}|z|^{2} / l_{0}^{2}\right)$. The wavefunction can then be rewritten as

$$
\Psi_{\frac{1}{2 m}}=\left[\prod_{i} e^{-\frac{1}{4}\left|z_{i}\right|^{2} / l_{B}^{2}}\right] \operatorname{Det}\left[e^{i \bar{k}_{j} z_{k}+i l_{B}^{2} k_{j} \frac{d}{d z_{k}}}\right] \prod_{i<j}\left(z_{i}-z_{j}\right)^{2 m}
$$

The operator $\exp \left(i l_{0}^{2} k \frac{d}{d z}\right)$ is then a shift operator for the position $z$ which takes $z \rightarrow z+i l_{0}^{2} k$. Thus, the wavefunction can be rewritten as ${ }^{63,58}$

$$
\Psi_{\frac{1}{2 m}}=\mathcal{A}\left[\prod_{i} e^{-\frac{1}{4}\left|z_{i}\right|^{2} / l_{B}^{2}+i \bar{k}_{i} z_{i}}\right] \prod_{i<j}\left(\left[z_{i}+i l_{B}^{2} k_{i}\right]-\left[z_{j}+i l_{B}^{2} k_{j}\right]\right)^{2 m}
$$

where $\mathcal{A}$ is the antisymmetrizing operator that sums over all possible pairings of the $z_{i}$ 's with the $k_{i}$ 's with odd permutations added with a minus sign. It is now clear that the effect of the fermionic determinant is to move the position of the zeros of the wavefunction away from the location of the electrons by a distance $l_{B}^{2} k$ which is given in terms of the "momentum" $k$. In order to minimize the Coulomb energy, the distances $l_{B}^{2} k_{j}$ should be minimized to keep the zeros of the wavefunction as close to the electrons as possible. Furthermore, in order for the wavefunction to be nonzero after antisymmetrization, all of the $k_{j}$ 's must be different so the lowest energy configuration should be a filled Fermi sea. What is interesting here is that the size of the Fermi sea must be minimized in order to minimize the potential energy, whereas for the free electron gas, the Fermi sea is minimized so as to minimize the kinetic energy. Surprisingly, boosting the entire Fermi sea via $\mathbf{k}_{i} \rightarrow \mathbf{k}_{i}+\mathbf{K}$ leaves the energy of the wavefunction (Eq. 182) completley unchanged! (the net effect of the transformation is to multiply the wavefunction by $\exp \left(i \bar{K} \sum_{j} z_{j}\right)$ which simply changes the center of mass wavefunction while keeping the center of mass of the system in the lowest Landau level thus costing no energy). This is quite unlike the case of noninteracting fermions. This invariance which we will call $\mathbf{K}$ invariance, will be extremely important below. We will see in section 7.7 below that preserving this symmetry is essential in understanding the behaviour of the composite Fermion fermi liquid in the wavefunction approach. In terms of a Fermi-Liquid theory, the statement that it costs no energy to boost the Fermi-sea is equivalent to saying that there is a Landau-Fermi liquid coefficient $F_{1}=m^{*} f_{1} /\left(2 \pi \hbar^{2}\right)=-1$ at least in the long wavelength low frequency limit (See Eq. 88). We will return to this issue in section 7.7.

The picture that we now have of the composite fermion at $\nu=\frac{1}{2 m}$ is that of an electron "bound" to an even number $(2 \mathrm{~m})$ of zeros of the wavefunction, which create its positively charged correlation-hole. The charge on the electron is of course -1 whereas there is a charge of $+\nu$ for each zero of the wavefunction, 
thus resulting in a total charge of $2 m \nu-1=0$, i.e., a neutral object. The zeros of the wavefunction are then boosted away a distance $l_{B}^{2}|k|$ from the precise location of the electron by the fermionic determinant which makes the wavefunction antisymmetric, resulting in a dipole moment of the electroncorrelation-hole pair. The Coulomb attraction between the electron and these zeros is some potential $V\left(l_{B}^{2}|k|\right)$. As usual in a high magnetic field, a force on a particle results in a drift motion of the particle perpendicular to the force and proportional to the gradient of the potential given by ${ }^{e}$

$$
v_{\mathrm{drift}}=\frac{c \nabla V \times \mathbf{B}}{q|\mathbf{B}|^{2}}
$$

where $q$ is the charge of the particle in question. Although the force on the correlation hole and the force on the electron are oppositely directed (directed towards each other) their drift velocities are in the same direction since the charge on the electron is opposite that of the hole. Thus, the electron-correlationhole pair drift in a direction perpendicular to their separation while maintaining a fixed separation. This is very reminiscent of the advective motion of a vortex-antivortex pair in fluid dynamics. Since the velocity of the pair is determined by the gradient of the potential $d V\left(l_{B}^{2}|k|\right) / d k$, we can think of $d^{2} V\left(l_{B}^{2}|k|\right) / d k^{2} \sim 1 / m^{*}$ as determining the effective mass of the pair ${ }^{58}$. Note that this effective mass is determined entirely by the interaction strength between the electron and correlation-hole.

\subsection{Excitations and Small $\Delta B$}

This trial ground state wavefunction can very naturally be extended to describe excited states. One simply chooses to replace the Fermi sea in Eq. 180 by an excited Fermi sea, by promoting one of the $k$-vectors from a value below the Fermi level to a value above the Fermi level. It has been shown numerically that these trial excited states have very good overlaps with the exact excited states of the system ${ }^{5,6,63}$.

We can also consider what happens to such an excitation when the magnetic field is not quite quite at $\nu=\frac{1}{2 m}$. Here, the total charge on the electroncorrelation-hole bound state is given by $e^{*}=-1+2 m \nu$ which is no longer zero owing to the fact that $\nu$ is no longer quite $\frac{1}{2 m}$. We might then expect such a charged object in a magnetic field to undergo cyclotron motion. Indeed this will turn out to be the case ${ }^{58}$. We once again consider the structure of the bound state of the electron and the correlation hole of $2 m$ zeros of

${ }^{e}$ Again, the drift velocity is such that the total force $\nabla V+\frac{q}{c} \mathbf{v} \times \mathbf{B}$ vanishes. 
the wavefunction. Since the charges on these two objects are no longer precisely opposite, the two drift velocities (Eq. 183) are slightly unequal with the electron velocity differing from the correlation-hole velocity by a factor of $q_{\text {hole }} / q_{\text {electron }}=(2 m \nu) /(-1)$. Because of this inequality in velocities, the pair will turn slowly as they move eventually completing a circle with the faster particle moving around the outside and the slower particle moving on the inside ${ }^{f}$. The ratio of radii of motion of these two particles $\left(R_{\text {electron }} / R_{\text {hole }}\right)$ is given by this same ratio of velocities (or ratio of charges), $2 m \nu$. The difference in radii, on the other hand, is given by the distance between the electron and its correlation-hole. Since we are interested in excitations near the Fermi surface, we can set $k \approx k_{\mathrm{F}}$, and the distance between the electron and its screening vortices is given by $R_{\text {electron }}-R_{\text {hole }}=2 l_{B}^{2} k_{\mathrm{F}}=l_{B} / \sqrt{m}$. With this information we can solve (with some algebra) to find that the radius of the cyclotron motion is (for $\nu$ near $\frac{1}{2 m}$ )

$$
R_{\text {electron }} \approx R_{\text {hole }} \approx \frac{k_{\mathrm{F}} \hbar c}{B e^{*}}=\frac{k_{\mathrm{F}} \hbar c}{e B(1-2 m \nu)}=\frac{k_{\mathrm{F}} \hbar c}{e \Delta B}
$$

The first form of this expression is what one might have expected in the first place - an object (the bound electron-correlation-hole pair or composite fermion) of charge $e^{*}=e(1-2 m \nu)$ should have a cyclotron radius given by $k_{\mathrm{F}} \hbar c /\left(e^{*} B\right)$. On the other hand, this result can also be equivalently rewritten in the form of a particle of charge $e$ in a reduced magnetic field $\Delta B$ so that it agrees with the Chern-Simons result in Eq. 21.

\subsection{Jain's Wavefunctions}

We would like to write down wavefunctions analogous to Eq. 180 for quantized Hall states that form near $\nu=\frac{1}{2 m}$. To describe a wavefunction at an arbitrary filling fraction $\nu$, we once again begin with the bosonic Laughlin state at filling fraction $\frac{1}{2 m}$ whose correlations are expected to be good, and we would like to "minimally damage" this wavefunction to make it an appropriate wavefunction for the new filling fraction. Here, we not only need to make the wavefunction antisymmetric, but we must also add enough zeros to the wavefunction to change the filling fraction from $\frac{1}{2 m}$ to the target filling fraction $\nu$. To this end, we consider the generalization of Eq. 180 constructed by replacing the fermionic determinant of plane wave states representing fermions in zero effective field $\left(\nu=\frac{1}{2 m}\right)$ with a wavefunction $\Phi_{p}$ for fermions in a finite effective

\footnotetext{
${ }^{f}$ Imagine a car whose left wheels move faster than its right.
} 
field ${ }^{g}$, Thus we obtain a wavefunction of the form

$$
\Psi_{\frac{p}{2 m p+1}}=\mathcal{P}_{\mathrm{LLL}} \Phi_{p}\left(\mathbf{r}_{1}, \mathbf{r}_{2}, \ldots, \mathbf{r}_{N}\right) \prod_{i<j}\left(z_{i}-z_{j}\right)^{2 m} \prod_{i} e^{-\frac{1}{4}\left|z_{i}\right|^{2} / l_{B_{1 / 2 m}}^{2}}
$$

where the wavefunction $\Phi_{p}$ is the wavefunction for free fermions at filling fraction $p$. Certainly, the resulting wavefunction is antisymmetric since the bosonic Laughlin state is symmetric and $\Phi_{p}$ is antisymmetric. Furthermore, it can be shown ${ }^{6}$ that the multiplication of the bosonic Laughlin state by the wavefunction $\mathcal{P}_{\text {LLL }} \Phi_{p}$ adds just enough zeros of the wavefunction to change the filling fraction from $\nu=\frac{1}{2 m}$ to $\nu=\frac{p}{2 m p+1}$. This should be easy to believe since we know that wavefunctions should have $B / \phi_{0}$ zeros per unit area. Thus multiplying a wavefunction corresponding to a field $B_{1}$ with a wavefunction corresponding to a field $B_{2}$ results in a wavefunction corresponding to a field $B_{1}+B_{2}$. Here, we have the bosonic Laughlin state corresponding to a field $B_{1 / 2 m}=\frac{n_{\mathrm{e}} \phi_{0}}{2 m}$ and the composite fermion wavefunction $\Phi_{p}$ corresponding to a field $\Delta B=n_{\mathrm{e}} \phi_{0} p$ which then add to yield a filling fraction $\nu=\frac{n_{\mathrm{e}} \phi_{0}}{B+\Delta B}=\frac{p}{2 m p+1}$. (The issue of projection makes the argument slightly more complicated than this simplified version).

Note also that the magnetic length in the exponential is taken to be the magnetic length at filling fraction $\nu=\frac{1}{2 m}$. There will in general be another exponential factor within the wavefunction $\Phi_{p}$ with magnetic length $l_{\Delta B}$. These two will combine together to yield an exponential with the proper magnetic length for a system in field $B=B_{1 / 2 m}+\Delta B\left(\right.$ since $\left.l_{B}^{-2}=l_{B_{1 /(2 m)}}^{-2}+l_{\Delta B}^{-2}\right)$.

The prescription outlined here for constructing wavefunctions at filling fraction $\nu=\frac{p}{2 m p+1}$ is of course precisely that first proposed by Jain ${ }^{6}$. It should be noted that, for the sake of pedagogy, we have arrived at the Jain wavefunctions only after a discussion of the $\nu=\frac{1}{2 m}$ state; whereas historically, Jain's wavefunction was introduced well before there was even interest in the even denominator states. It should further be noted that Rezayi-Read wavefunction is clearly just the $p \rightarrow \infty$ limit of the Jain wavefunction.

For $p$ an integer, the wavefunction $\Phi_{p}$ can be taken as a trivial fermionic determinant of $p$ filled Landau levels. As an example, we consider the $p=1$ case, corresponding to $\nu=\frac{1}{2 m+1}$. Here we have

$$
\Psi_{\frac{1}{2 m+1}}\left(z_{1}, z_{2}, \ldots, z_{N}\right)=\left[\prod_{i<j}\left(z_{i}-z_{j}\right) \prod_{i} e^{-\frac{1}{4}\left|z_{i}\right|^{2} / l_{\Delta B}^{2}}\right] \times
$$

\footnotetext{
${ }^{g}$ As discussed above, we could equivalently think of this as free fermions of charge $1-2 m \nu$ in the full magnetic field $B$. The effective filling factor $p$ will remain the same.
} 


$$
\left[\prod_{i<j}\left(z_{i}-z_{j}\right)^{2 m} \prod_{i} e^{-\frac{1}{4}\left|z_{i}\right|^{2} / l_{B_{1 / 2 m}}^{2}}\right]
$$

The first factor is just the wavefunction for a single filled Landau level $\Phi_{p=1}$. The second factor (with the power $2 m$ ) is the composite-fermionization factor that attaches $2 m$ zeros of the wavefunction to each electron. Thus, the $\nu=$ $\frac{1}{2 m+1}$ state is written as a composite fermionized $\nu=1$ state. As discussed above, the two exponential factors will combine to give the proper exponential factor for the full magnetic field, and we will be left with simply the Laughlin wavefunction (Eq. 178). We note that the composite fermion quasiparticle - the electron plus its vortex screening cloud of $2 m$ extra zeros - has a net charge of $e^{*}=-1+2 m \nu$ which is the correct quasiparticle charge, $-\frac{1}{2 m+1}$ for the quasiparticle of the Laughlin state.

In this case of $p=1$, where we recover the Laughlin wavefunction, the projection is not needed here since the wavefunction $\Phi_{p=1}$ is already in the lowest Landau level. For $p$ not equal to 1, we will need to project the resulting wavefunction back down to the lowest Landau level, and the final form of the wavefunction will (after projection) be quite complex (See for example, Ref. $64)$. Nonetheless, it has been shown numerically that such a prescription results in a trial wavefunction with extremely high overlap with the exact ground state for small systems ${ }^{5,6}$.

The composite fermionization described by Eq. 185 or Eq. 180, can be thought of in several equivalent ways. On the one hand, we can think of the composite-fermionization as adiabatically inserting $2 m$ flux quanta at the position of each electron. Equivalently we can think of this factor as the binding of $2 m$ positively charged zeros (vortices) to each electron. The good energetics of these composite fermion states can be thought of in terms of the large binding energy ${ }^{57}$ of these electron-correlation-hole objects (which are, of course, the composite fermions themselves).

\subsection{Wavefunctions vs. Chern-Simons Theory}

The idea of the composite fermionization factor being equivalent to adiabatically inserting $2 m$ flux quanta at the position of each electron is certainly quite reminiscent of the Chern-Simons transformation described in section 2.2 above. To make the relation more clear we rewrite the Chern-Simons transformation (Eq. 10) in analytic coordinates as

$$
\Psi_{\mathrm{e}}\left(\mathbf{r}_{1}, \mathbf{r}_{2}, \ldots, \mathbf{r}_{N}\right)=\left[\prod_{i<j}\left(\frac{z_{i}-z_{j}}{\left|z_{i}-z_{j}\right|}\right)^{2 m}\right] \Phi\left(\mathbf{r}_{1}, \mathbf{r}_{2}, \ldots, \mathbf{r}_{N}\right) .
$$


If we consider the $\nu=\frac{1}{2 m}$ state, at the mean field level, the composite fermion wavefunction $\Phi$ is just a filled Fermi sea (Eq. 179). We then see that the Chern-Simons transformed wavefunction $\Psi_{\mathrm{e}}$ looks very similar to the RezayiRead trial wavefunction (Eq. 180). As with the Rezayi-Read wavefunction, an extra phase factor of $e^{2 m \pi i}$ is included when one electron wraps around another. However, in the Chern-Simons transformation, there is a non-analytic factor $\left|z_{i}-z_{j}\right|$ in the denominator which indicates that the wavefunction (Eq. 187) is not properly in the lowest Landau level. However, we must remember that choosing $\Phi$ to be the filled Fermi sea is just the mean field result of the ChernSimons theory. When the leading fluctuations around mean field are accounted for, it can be shown that the non-analytic factor in the denominator is canceled, at least when one is only concerned with the long distance physics ${ }^{66,49,15}$. However, even after this cancellation, the factor $\Phi$ may retain pieces outside of the lowest Landau level. Obtaining a fully projected Fermi sea within a Chern-Simons approach remains an open problem.

Another interesting difference between the wavefunction approach and the Chern-Simons approach is that in the wavefunction approach, the elementary excitation, the bound electron-correlation-hole has charge $(1-2 m \nu) e$ with a dipole moment whereas in the Chern-Simons approach the transformed fermion has charge $e$ with no obvious dipole moment. However, as discussed above, many of the important physically relevant parameters like the cyclotron radius and the effective filling fraction away from $\nu=\frac{1}{2 m}$ turn out the same in either approach.

Finally, we note that in the wavefunction approach, there is no ChernSimons gauge field. Thus, one might wonder if the unusual infrared problems of the gauge-field propagator might be absent in the dipole-fermion picture of the composite fermion. We will see below that these infra-red problems re-appear in a slightly different guise.

\subsection{Response of Neutral Dipole Composite Fermions}

Several recent approaches have attempted to bring these two approaches into more of an agreement. D.-H. Lee ${ }^{51}$ has constructed a Chern-Simons theory where particles and holes bind together to form neutral fermions in a manner more reminiscent of the above wavefunction arguments. Work by Shankar and Murthy ${ }^{49}$ separates out the inter-Landau-level excitations and results in a field theory that is somehow "projected" to the lowest Landau level. Finally, Haldane and Pasquier ${ }^{50}$ have used a lowest Landau level algebraic approach. These three approaches all seem to be converging on a coherent picture of

a Fermi sea of neutral dipoles at $\nu=\frac{1}{2}$. The neutral-dipole Hamiltonians 
obtained by all of these groups appear to be equivalent (at least as long as one is only concerned with the long distance physics). In this section, we will show (at least roughly) how these approaches lead to the same results as that of the Chern-Simons approach.

One natural question to ask is how can we have any DC current if all of the particles in the system are neutral? As we discussed in section 2.1.1 above, by Galilean invariance, any clean system must have a DC Hall conductivity $\sigma_{x y}=\nu \frac{e^{2}}{h}$, so we know that current must be carried somehow. This strange contradiction is a result of having restricted our attention to the lowest Landau level ${ }^{65}$, which by itself is not Galilean invariant. Application of an electric field to the system mixes Landau levels (no matter how large $\omega_{\mathrm{c}}$ is) and it is precisely these higher Landau level pieces that carry the current ${ }^{h}$. Thus, in order to obtain the proper Hall conductivity, we must include these Landau level excitations, which complicates matters (see Ref. 49 for a discussion of this physics). Here, we will instead focus on the response $K_{00}(\mathbf{q}, \omega)$ at frequencies well below the cyclotron frequency which can be obtained without consideration of these higher energy excitations.

We will now give a slightly hand-waving derivation of the electronic response in the neutral dipole picture. Note that we will call these fermionic objects "dipole fermions" even at filling fractions away from $\nu=\frac{1}{2 m}$ when they have a charge as well as a dipole moment.

\subsubsection{Dipole Fermion Variables}

Inspired by the above discussion, we write the electron density $(\rho)$ in the form ${ }^{49}$

$$
\rho=e^{*} \rho^{\mathrm{d}}-i l_{B}^{2} \nabla \times \mathbf{g}
$$

where $\rho^{\mathrm{d}}(\mathbf{r})=\sum_{i} \delta\left(\mathbf{r}-\mathbf{r}_{i}\right)$ is the dipole fermion density and

$$
\mathbf{g}(\mathbf{r})=\sum_{i}\left[\mathbf{p}_{i}+\frac{e^{*}}{c} \mathbf{A}\left(\mathbf{r}_{i}\right)\right] \delta\left(\mathbf{r}-\mathbf{r}_{i}\right)
$$

is the dipole fermion momentum density (We use the notation $\mathbf{g}$ rather than writing this as a current $\mathbf{j}$ because we do not want the factor of mass to enter here). Here, $\mathbf{r}_{i}$ and $\mathbf{p}_{i}$ are the position and momentum of the $i^{\text {th }}$ dipole fermion (which are assumed to obey canonical commutation relations). The

\footnotetext{
${ }^{h}$ It may seem strange that we can mix Landau levels even for large $\omega_{\mathrm{c}}$. Indeed, as we take $m_{\mathrm{b}} \rightarrow 0$ such that $\omega_{\mathrm{c}} \rightarrow \infty$, the amount of mixing gets small. However, the current operator $\mathbf{j}=\mathbf{p} / m_{\mathrm{b}}$ has a factor of $m_{\mathrm{b}}^{-1}$ which compensates so that the current due to this mixing remains finite.
} 
first term in Eq. 188 is just the density of these fermions times their net charge $e^{*}=-1+2 m \nu$ which, of course, vanishes at filling fraction $\frac{1}{2 m}$ where the fermions become neutral dipoles. The second term is the dipolar term that gives each fermion a dipole moment perpendicular to its momentum as suggested by the above discussion. In that term $e^{*} \mathbf{A}=e \Delta \mathbf{A}$ is the vector potential associated with the field difference from the $\nu=\frac{1}{2 m}$ denominator state.

We note that this form of the electron density has the appealing feature that, in the long wavelength limit, it satisfies the proper commutation relations for a density in the lowest Landau level ${ }^{49}$ given by ${ }^{62}$

$$
\left[\rho(\mathbf{q}), \rho\left(\mathbf{q}^{\prime}\right)\right]=i l_{B}^{2}\left(\mathbf{q} \times \mathbf{q}^{\prime}\right) \rho\left(\mathbf{q}+\mathbf{q}^{\prime}\right)
$$

where we have expanded for small $q$ here. Since this representation of $\rho$ satisfies the proper commutation, it is completely acceptable to work in terms of these dipole fermion variables (at least for small $q$ ). However, the dipole variables have 2 degrees of freedom per particle whereas the lowest Landau level has only one. There must therefore be constraints on these degrees of freedom. These constraints have been written down but it is not at all clear how to properly handle them in a systematic way ${ }^{49-51}$ which makes performing a completely controlled calculation difficult. The simplest thing we can do is to neglect the constraint entirely which we will do here.

For simplicity, we will now focus on filling fraction $\frac{1}{2 m}$ where the density becomes

$$
\rho(\mathbf{q})=-i \frac{\nu}{2 \pi n} q g_{1}(\mathbf{q})=-i l_{B}^{2} \sum_{i}\left(\mathbf{q} \times \mathbf{p}_{i}\right) e^{-i \mathbf{q} \cdot \mathbf{r}_{i}}
$$

with $g_{1}=\hat{\mathbf{q}} \times \mathbf{g}$ the transverse part of the momentum current of the dipole fermions. Here, the dipole fermions see no effective magnetic field (since they are neutral). For a discussion of $\nu \neq \frac{1}{2 m}$ see Refs. 49 and 53. We note here that we have $\rho \sim \nabla \times \mathbf{g}$ which appears quite similar to the Chern-Simons case $\rho \sim \nabla \times$ a with a the Chern-Simons vector potential. These two actually act in quite similar ways as we will see below.

The form of Eq. 191 has been explicitly derived by several groups ${ }^{49-51}$. Here, however, we have neglected the contribution of high energy "magnetoplasmon" oscillations (inter Landau-level excitations). As mentioned above, several properties of the system, such as the Hall conductivity, cannot be correctly obtained without considering the contributions of these high energy excitations $^{49,65}$. However, for calculating the response $K_{00}$, we can stay completely in the lowest Landau level. 


\subsubsection{Response Functions}

We now wish to calculate the density-density response function $K_{00}$ for electrons. From linear response theory, we know that $K_{00}(\mathbf{q}, \omega)$ is given by the Fourier transform of the time ordered correlator (See Eqs. 141 and 143 with $\left.E_{00}=0\right)$

$$
K_{00}(\mathbf{r}, t)=\langle T \rho(\mathbf{r}, t) \rho(0,0)\rangle
$$

Using Eqs. 191 and 192, we relate this to a correlator for the dipole fermion current

$$
K_{00}(\mathbf{q}, \omega)=\left(\frac{\nu q}{2 \pi n}\right)^{2} D_{11}^{\mathrm{d}}(\mathbf{q}, \omega)
$$

where we have defined $D_{11}^{\mathrm{d}}(\mathbf{q}, \omega)$ to be the Fourier transform of the time ordered transverse momentum correlator of the dipole fermions

$$
D_{11}^{\mathrm{d}}(\mathbf{r}, t)=\left\langle T g_{1}(\mathbf{r}, t) g_{1}(0,0)\right\rangle
$$

(Again, we have the notation that $g_{1}$ is the transverse part of g. Also note that the usually the current correlator [See Eq. 141] is defined with respect to the current $\mathbf{j}$ rather than the momentum current $\mathbf{g}$ which differ by factors of mass). We note that here, this dipole correlator is precisely equivalent to the transverse gauge field propagator $\mathcal{D}_{11}$ we found in Eq. 150 (compare Eq. 193). Thus, our choice of notation of using $D^{\mathrm{d}}$ for the dipole current correlator, and $\mathcal{D}$ as the gauge field correlator is conveniently chosen to be suggestive that they play the same role.

Analogous to the usual separation of the response $K_{00}$, into a polarization and a Hartree long range interaction, we now define the correlator $\tilde{D}_{11}^{\mathrm{d}}$, which is the part of $D_{11}^{\mathrm{d}}$ which is irreducible with respect to the long range Coulomb interaction between the dipoles. Being that the dipole-dipole interaction is written as

$$
\begin{aligned}
H_{\mathrm{int}} & =\sum_{q} v(q) \rho^{e}(\mathbf{q}) \rho^{e}(-\mathbf{q}) \\
& =\sum_{q} v(q)\left(\frac{q \nu}{2 \pi n}\right)^{2} g_{1}(\mathbf{q}) g_{1}(-\mathbf{q})
\end{aligned}
$$

we define the irreducible correlator via

$$
\left[D_{11}^{\mathrm{d}}\right]^{-1}=\left[\tilde{D}_{11}^{\mathrm{d}}\right]^{-1}-v(q)\left(\frac{q \nu}{2 \pi n}\right)^{2}
$$

More generally, these correlators should be treated as matrices $D_{\mu \nu}^{\mathrm{d}}$. However, at filling fractions $\nu=\frac{1}{2 m}$ these matrices are all diagonal and we can 
consider one component at a time ${ }^{i}$ (see Ref. 53 for $\nu \neq \frac{1}{2 m}$ ). We note that this Hartree separation of the dipole response can be compared to Eqs. 48 and 193 to show that the electronic polarization is given by

$$
\Pi_{00}^{v}(\mathbf{q}, \omega)=\left(\frac{\nu q}{2 \pi n}\right)^{2} \tilde{D}_{11}^{\mathrm{d}}(\mathbf{q}, \omega) .
$$

In other words, one can equivalently treat the Coulomb interaction in terms of its action on the original electrons or in terms of its action on the dipole fermions. It is interesting to note that although the Coulomb interaction looks very weak when written in terms of the dipoles, it is equally important independent of which variables it is written in terms of.

We note that the importance of the irreducible correlator $\tilde{D}^{\mathrm{d}}$ is that at the RPA level (as in a Landau-Silin theory) we will be able to use a fully local Hamiltonian to calculate this quantity while neglecting the long range part of the interaction.

\subsubsection{K-invariance}

One might at this point be tempted to naively approximate the dipole fermions as noninteracting fermions ${ }^{j}$. In the long wavelength, low frequency limit, the correlator $D_{11}^{0}$ for noninteracting fermions is a constant (See Eq. 203 below). Approximating either $D_{11}$ (or more properly $\left.\tilde{D}_{11}\right)$ as a constant $\left(D_{11}^{0}\right)$ would lead us to naively believe (Using Eq. 193) that the electron response is given by $K_{00} \sim q^{2}$ which differs drasticly from the Chern-Simons RPA results obtained in section 3 and 4 above. The apparent reason for this "incompressibility" is that the external fields couple very weakly to the dipole particles ${ }^{k}$ This apparent contradiction with the result of Chern-Simons theory has been the source of much confusion in the community.

The error in this naive approximation is that, as discussed in section 7.3 above, the dipoles represented by the Rezayi-Read $\nu=\frac{1}{2}$ wavefunction form a very unusual Fermi liquid that is invariant under the transformation $\mathbf{p}_{i} \rightarrow$ $\mathbf{p}_{i}+\mathbf{K}$ which corresponds to a boost of the Fermi sea. In Ref. 53 it is shown that within the formalism of Shankar and Murthy, this so-called K-invariance is actually a gauge symmetry. Since it costs no energy to boost the system uniformly, we will find that the current response $D_{11}^{\mathrm{d}}$ diverges in the long wavelength limit canceling powers of $q$ in Eq. 193 (or Eq. 198) so that the

\footnotetext{
${ }^{i}$ So long as we continue to ignore all constraints.

${ }^{j}$ Such an approximation has been made in various early versions of Refs. $49-51$. See Refs. 52 and 53 for a detailed discussion of how such an approximation fails.

${ }^{k}$ A quantized Hall state has $K_{00} \sim q^{4}$ whereas a compressible state has $K_{00} \sim 1 / v(q)$ whenever $v(q)$ diverges for small $q$ and $K_{00} \sim$ constant otherwise.
} 
system remains compressible $\left(\Pi_{00} \sim\right.$ constant or $\left.K_{00} \sim 1 / v(q)\right)$ in agreement with the predictions of the Chern-Simons theory.

Approximating the dipole particles as noninteracting fermions clearly violates this $\mathbf{K}$-invariance since it certainly costs kinetic energy to boost an entire Fermi sea of noninteracting fermions. We again turn to Fermi liquid theory for a proper description of this deviant Fermi liquid. In the Landau-Silin sense, we have already separated out the long range part of the interaction in Eq. 197 and we should now describe the response $\tilde{D}^{\text {d }}$ (analogous to $\Pi$ ) in terms of a Landau Fermi liquid theory with only local interactions (this is all discussed in depth in section 4 above). Thus, in a Landau Fermi liquid description of these dipole fermions, we should think of the dipoles as having some mass $m^{*}$ and some Landau interaction parameters $F_{n}$ (See section 4 above). In order to guarantee that it costs no energy to boost the whole Fermi sea all we need to do is to force $F_{1}=-1$ (See Eq. 88) at least in the long wavelength limit ${ }^{l}$.

To see how the value of the Landau parameter $F_{1}=-1$ preserves this $\mathbf{K}$-symmetry, we note that the $F_{1}$ interaction is a current-current interaction (See Eqs. 88, 86 and 87 above). A local Hamiltonian representing fermions with mass $m^{*}$ interacting via an $F_{1}$ interaction is written as

$$
H_{\text {local }}=\sum_{i} \frac{\mathbf{p}_{i}^{2}}{2 m^{*}}+\frac{F_{1}}{2 n m^{*}} \int d \mathbf{r}|\mathbf{g}(\mathbf{r})|^{2}
$$

where $\mathbf{g}$ is the momentum density (Note that this differs slightly from the discussion above in section 4 because factors of the mass are left out of $\mathbf{g}$ whereas conventionally we put these factors in and call the current $\mathbf{j}$ ). For the value of $F_{1}=-1$, this local Hamiltonian is equivalent to the form proposed by Haldane ${ }^{63}$,

$$
H_{\text {local }}^{\left(F_{1}=-1\right)}=\frac{1}{2 m^{*}} \sum_{i}^{N}\left(\mathbf{p}_{i}-\frac{1}{N} \sum_{j}^{N} \mathbf{p}_{j}\right)^{2}
$$

which is clearly $\mathbf{K}$-invariant. The physical meaning of this expression is that the momentum of each fermion is measured with respect to the average momentum.

\footnotetext{
${ }^{l}$ Examining Eq. 90 we see that for fixed effective mass $m^{*}$, the value $F_{1}=-1$ implies that $m_{\mathrm{b}} \rightarrow \infty$. This is clearly not the true bare mass of the problem (which we have taken to zero!). This "effective" infinite bare mass makes sense because it should cost zero energy to boost the Fermi sea by momentum $\mathbf{K}$ yielding an energy cost $\mathbf{K}^{2} /\left(2 m_{\mathrm{b}}\right)$. We note that this divergence of the "effective" bare mass is the reason we are working with the momentum density $\mathbf{g}$ rather than the current $\mathbf{j}=\mathbf{g} / m_{\mathrm{b}}$.
} 


\subsubsection{Dipole RPA}

We would now like to apply the RPA to this effective local interaction to obtain a physical response function. With the experience gained in section 3 and 4 above we know quite well how to treat the momentum-momentum interaction in the local dipole Hamiltonian (Eq. 199). We separate out the interaction term and define an irreducible response $D_{11}^{\text {irr }}$ given by

$$
\left[\tilde{D}_{11}^{\mathrm{d}}\right]^{-1}=\left[\tilde{D}_{11}^{\mathrm{irr}}\right]^{-1}+\frac{F_{1}}{n m^{*}}
$$

Note that this separation of $F_{1}$ is equivalent to Eq. 100.

The RPA approximation is now just the statement that $\tilde{D}_{11}^{\text {irr }}$ should be approximated as the momentum correlator of noninteracting fermions. We now recall that the transverse momentum correlator $D_{11}^{0}$ is closely related to the a response function $K_{11}^{0}$ of free fermions (See Eq. 143). Note that here $D^{0}$ is a correlator of momentum current rather than charge current so there are two extra factors of mass compared to Eq. 143. As discussed in section 6.2 above, for noninteracting fermions of mass $m^{*}$ we have the relation (accounting for the extra mass factors)

$$
K_{11}^{0 *}(\mathbf{q}, \omega)=\frac{1}{\left(m^{*}\right)^{2}} D_{11}^{0 *}(\mathbf{q}, \omega)-\frac{n}{m^{*}} .
$$

The ${ }^{*}$ again indicates the mass is renormalized. Using the low frequency form of $K^{0}$ given in Appendix A, yields the momentum correlator for noninteracting fermions of mass $m^{*}$,

$$
D_{11}^{0 *}(\mathbf{q}, \omega)=n m^{*}+\frac{q^{2} m^{*}}{24 \pi}+i \frac{2 n \omega\left(m^{*}\right)^{2}}{k_{\mathrm{F}} q}+\ldots
$$

We now make the RPA and approximate the irreducible correlator $\tilde{D}^{\text {irr }}$ as $D^{0 *}$. It is critical to note that in Eq. 201 the leading term in $D^{0 *}$ cancels the interaction term for $F_{1}=-1$. This precise cancellation leads to $\tilde{D}_{11}^{\mathrm{d}} \sim q^{-2}$ in the low frequency limit which is singular and cancels the powers of $q$ in Eq. 198 resulting in a polarization $\Pi_{00}^{v}$ which is constant in the low frequency long wavelength limit representing a compressible system as expected. Carrying out this calculation explicitly using Eqs. 203, 201, 197 and 193 yields the low frequency, long wavelength response

$$
K_{00}=\frac{q^{2}}{\left(\frac{2 \pi}{\nu}\right)^{2}\left[\frac{q^{2}}{24 \pi m^{*}}-i \frac{\omega k_{\mathrm{F}}}{2 \pi q}\right]+q^{2} v(q)}
$$


At higher frequencies (but still well below the cyclotron energy) we can write our result in terms of the response $K^{0 *}$ of noninteracting fermions of mass $m^{*}$ (using Eq. 202) yielding

$$
K_{00}=\frac{1}{\left(\frac{2 \pi}{q \nu}\right)^{2}\left[\left(\left[K_{11}^{0 *}\right]^{-1}+\frac{m^{*}}{n}\right)^{-1}\right]+v(q)}
$$

We can compare this result to the result of the Modified RPA calculation of Eq. 108 in the limit of $m_{\mathrm{b}} \rightarrow 0$ (i.e., if we make the cyclotron energy very large so we can focus on lowest Landau level behavior). We see that these results are quite similar although one finite $q$ correction term related to $K_{00}^{0}$ seems to be present in the MRPA calculation (or in HLR) which is not present in this dipole approach. It is believed that this correction term can also be obtained in this dipole picture ${ }^{53}$ to obtain complete agreement with the MRPA by imposing the constraint that $\rho^{\mathrm{d}}=\left(q m^{*} / \omega\right) g_{x}=\rho^{e}$, which then imposes a relation (via Eq. 191) between the transverse and longitudinal parts of $\mathbf{g}$. This allows the transverse current of $\mathbf{g}$ to couple to the compression mode of the dipoles, hence producing the appropriate factor of $K_{00}^{0}$. However, the details of this are not yet fully established (See Ref. 53).

We note however that the correction term vanishes in the limit of $q, \omega \rightarrow 0$, and whether or not we include it, we always obtain a form of low frequency, long wavelength, electron response given by

$$
K_{00} \sim \frac{1}{C_{1} \frac{i \omega}{q^{3}}+\left[C_{2}+v(q)\right]}
$$

that agrees with the form (Eq. 68) found by HLR (with $C_{1}$ and $C_{2}$ dimensionful constants). This form guarantees the existence of the overdamped relaxation characteristic of the HLR theory of the $\nu=\frac{1}{2}$ state. This overdamped mode also guarantees that we will encounter infra-red divergences when we try to calculate perturbative corrections to quantities such as the fermion effective mass. To see how this happens we note that the interaction term $F_{1}$ (as well as the dipole interaction $q^{2} v(q)$ ) provides our theory with an interaction vertex with the transverse current. When we calculate perturbative corrections, we should use $D_{11}^{\mathrm{d}}$ as a transverse current propagator. We can then calculate the self energy of the dipole fermion by writing the diagram shown in Eq. 154 only using $D_{11}^{\mathrm{d}}$ as a propagator rather than the Chern-Simons gauge field propagator $\mathcal{D}_{11}$. But these two propagators are precisely the same (as required by Eq. 193 and 150). Thus, we obtain the exact same divergent diagrams as in the Chern-Simons approach. 
In summary, we have found that the picture of the dipole Fermi liquid seems to be equivalent to that of the Chern-Simons Fermi liquid. The picture we obtain from the two models are quite different, but at the end of the day the physical predictions turn out to be the same.

\section{Selected Experiments}

One of the most exciting features of composite fermion physics is that it is

a rich world for experimentalists as well as theorists. Literally hundreds of experiments have been performed in the composite fermion regime. For a more a thorough discussion of all of the experiments, see Ref. 24, as well as the chapters by Willett and Smet in this book.

\subsection{Surface Acoustic Waves}

The Surface Acoustic Wave experiments of Willett et al ${ }^{67}$ were the first clear indication of the existence of a compressible state at $\nu=\frac{1}{2}$. The theory of Halperin, Lee, and Read was then developed in close conjunction with the development of these experiments ${ }^{68}$. Since these experiments have been instrumental in the development of our understanding of $\nu=\frac{1}{2}$ physics, we will give a brief description of how this experiment measures the response $K_{00}$.

In the experiment, one applies a surface acoustic wave (SAW) to the surface of the sample which couples piezoelectrically to the $2 \mathrm{DEG}$ which is only slightly below the surface. The rough idea is that the SAW can excite modes in the 2DEG, thereby losing energy to the electron. By measuring the attenuation and velocity shift, one can extract information about the response of the 2DEG at the frequency and wavevector of the SAW.

To see more explicitly how this happens ${ }^{69}$, we consider a SAW of wavevector $q$, frequency $\omega=v_{s} q$ with $v_{s}$ the wave velocity, and amplitude $I$. The electric potential applied to the 2DEG due to the piezoelectric coupling is $A_{0}^{\text {ext }}=\zeta(q d) I$ where $d$ is the depth of the 2DEG beneath the surface, and $\zeta$ is some function related to the piezoelectric coupling, which is usually assumed to be roughly constant for $q d<4$ (See, however, Ref. 69). The induced density modulation is then $j_{0}=K_{00} A_{0}^{\text {ext }}=\zeta K_{00} I$ where, of course, all quantities are at $q$ and $\omega=v_{s} q$. The induced density interacting with the externally applied field results in an interaction energy density per unit area given by $\delta U=\frac{1}{2} j_{0} A_{0}^{\text {ext }}=\frac{1}{2} \zeta I^{2} K_{00}$. The mechanical energy density per unit area of the surface, on the other hand, is given by $U=\gamma q I^{2}$ with $\gamma$ some materials dependent constant (note that a wave has energy density $q^{2} I^{2}$, then we integrate to a depth of roughly one wavelength away from the surface to get an areal 
energy density proportional to $q$ ). Thus, the fractional energy shift is given by

$$
\frac{\delta U}{U}=\frac{\zeta(q d)}{2 \gamma q} K_{00}\left(q, \omega=v_{s} q\right)
$$

This fractional energy shift can be thought of as the fractional shift in frequency of the SAW. Thus, the real part is the fractional velocity shift, and the imaginary part will be the attenuation. We typically measure the velocity shift with respect to the shift that occurs for a system with infinite conductivity. For such an infinitely conductive system, (See Eqs. 48 and 56) we have $K_{00}=1 / v(q)$. Thus, with respect to that system as a reference,

$$
\frac{\delta v_{s}}{v_{s}}+i \frac{\kappa}{q}=\frac{\alpha^{2}}{2}\left[v(q) K_{00}\left(q, \omega=v_{s} q\right)-1\right]
$$

where $\kappa$ is the attenuation (In practice, the attenuation is difficult to measure accurately). Here we have made the conventional definition that $\alpha^{2} / 2=$ $\zeta(q d) /(2 \gamma q v(q))$. The coupling constant $\alpha$, which is a materials dependent function, ${ }^{a}$ is usually taken to be roughly constant for $q d<4$.

We now use Eq. 48, as well as Eq. 56 to write

$$
K_{00}=\frac{\epsilon q /(2 \pi)}{1-i \sigma_{\mathrm{m}} / \sigma_{x x}}
$$

with

$$
\sigma_{\mathrm{m}}=\frac{v_{s}}{v(q) q}
$$

For the Coulomb form of the interaction, $\sigma_{\mathrm{m}}=v_{s} \epsilon /(2 \pi)$ (Note, however, $\epsilon$ is weakly dependent on $q d$ since the 2 DEG is near a dielectric interface $\left.{ }^{69}\right)$. We can now insert this expression into Eq. 208 to obtain the often quoted result ${ }^{69,70}$

$$
\frac{\delta v_{s}}{v_{s}}+i \frac{\kappa}{q}=\frac{\alpha^{2} / 2}{1+i \sigma_{x x} / \sigma_{\mathrm{m}}} .
$$

where $\sigma_{x x}$ is evaluated at $q$ and $\omega=v_{s} q$.

In experiments by Willett ${ }^{67,68}$, it is found that the measured surface wave velocity shift at long wavelength do indeed reflect the DC conductivity $\sigma_{x x}$ of the 2DEG via this formula. However, in order to obtain a good match between the DC conductivity and the velocity shift, $\sigma_{\mathrm{m}}$ is treated as a fitting

\footnotetext{
${ }^{a}$ For GaAs samples with Coulomb interactions unscreened by a gate, $\alpha \approx 3.2 \times 10^{-6}$. However, this coupling depends somewhat ${ }^{69}$ on the depth of the 2DEG beneath the surface of the sample.
} 
parameter that may be as large as 4 times that predicted by Eq. 210. The reason for this discrepancy is unknown. One possible explanation is that long scale inhomogeneities in the system make the macroscopic DC conductivity not accurately reflect the microscopic conductivity of the system on the scale of the wavelength of the $\mathrm{SAW}^{71}$. Another possibility is that parallel conduction in the donor layer of the samples screens the Coulomb interaction, thus altering $v(q)$ and hence $\sigma_{\mathrm{m}}$ via Eq. 210. Until these details are sorted out, we should then probably treat $\sigma_{\mathrm{m}}$ as a fitting parameter.

Since we have prescriptions for calculating response functions for the composite fermion system, we should be able to convert this into a prediction for the surface wave velocity shift. We use Eq. 31 to write $\sigma_{x x}$ of the electrons in terms of $\rho_{y y}^{\mathrm{CF}}$ of the transformed fermions. Using a Landau-Boltzmann approach as discussed in section 4 above, for $\nu=\frac{1}{2 m}$, Eq. 104 gives us an expression for $\rho_{y y}$ where we have included an $F_{1}$ coefficient and current conserving scattering ${ }^{b}$ For $q \gg 1 / l$ (with $l=v_{\mathrm{F}}^{*} \tau$ a scattering length) we obtain $\sigma_{x x}\left(q, \omega=q v_{s}\right)=C q$ with $C$ a constant which is mostly real for $v_{s} \ll v_{\mathrm{F}}^{*}$. This is agreement with our earlier result Eq. 35. This results in a prediction of

$$
\frac{\delta v_{s}}{v_{s}} \approx \frac{\alpha^{2} / 2}{1+\tilde{C}^{2} q^{2}}
$$

where $\tilde{C}$ must be treated as unknown due to our uncertainty in the appropriate value of $\sigma_{\mathrm{m}}$. Such a form of the velocity shift is indeed in agreement with experiment ${ }^{68}$ for $q>1 / l$. It should be noted, however, that the current experimental data could also be in agreement with $\delta v_{s} / v_{s} \sim q$ for larger $q$. At lower $q$ where disorder is more important, $\sigma_{x x}$ is seen to roll over be a constant (See Eq. 36).

A more impressive agreement of theory with experiment ${ }^{72,73}$ occurs when one looks at finite $\Delta B$. Resonances seen at finite $\Delta B$ roughly indicate the commensuration of the SAW wavelength with the composite fermion cyclotron diameter $2 R_{\mathrm{c}}^{*}$. Using a Landau-Silin Boltzmann approach (called the "Modified Semiclassical" Approach above in section 4.2) with a current conserving scattering term as discussed in section 4 above, one can obtain predictions for the SAW velocity shift near $\nu=\frac{1}{2}$ that agree quite well with experiments ${ }^{74,37}$. There are several free parameters that must be put into the theory by hand here, including the effective mass $m^{*}$, the scattering time $\tau$, the constant $\sigma_{\mathrm{m}}$, as well as the fractional density inhomogeneity of the sample (which has the effect of smearing the magnetic field axis since the value of the filling fraction

\footnotetext{
${ }^{b}$ As mentioned above, it actually does not matter whether or not we worry about making sure the the scattering conserves current.
} 
differs slightly from place to place). However, making a reasonable guess for $\tau$ and the fractional density fluctuations (which can be roughly measured by other experiments) and using a theoretical prediction for $\sigma_{\mathrm{m}}$, we can make predictions for the SAW velocity shifts at various values of the effective mass which are shown in Fig. 3. Note, however, that uncertainties in $\sigma_{\mathrm{m}}$ will mainly have the effect of changing the scale of the vertical axis. (These figures use parameters appropriate to compare with the experimental data from Ref. 73). We see that up $m^{*} \approx 15 m_{\mathrm{b}}$ the predicted curves are relatively insensitive to the exact value of $m^{*}$. Above about $20 m_{\mathrm{b}}$, the location of the resonance and the shape of the curve changes dramatically as the SAW frequency actually moves above the lowest excitation mode of the system. Thus, we can put an upper bound on the value of the effective mass.

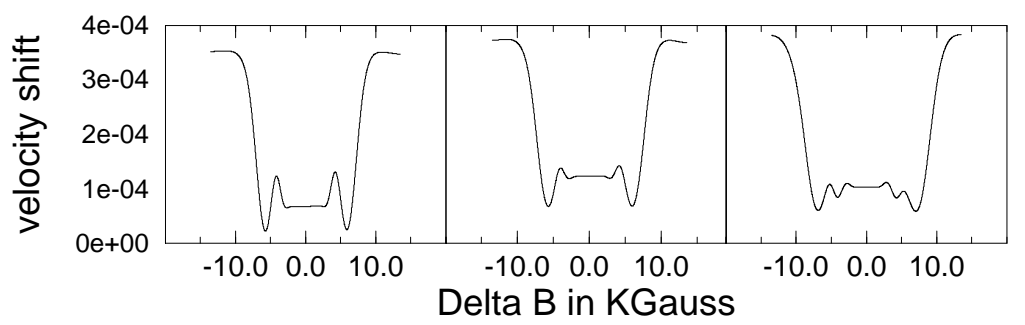

Figure 3: Predicted SAW velocity shifts for various values of the effective mass. From left to right $m^{*}=5 m_{\mathrm{b}}, 10 m_{\mathrm{b}}$, and $25 m_{\mathrm{b}}$, and Parameters are $n=1.64 \times 10^{11}$, frequency $=10.7 \mathrm{GHz}$, scattering length $l=1 \mu$, density inhomogeneity $=1 \%$, and $\sigma_{\mathrm{m}}=6.8 \times 10^{-7} \Omega^{-1}$. These are chosen to agree with the experimental work of Ref. 73 . One should think of the $y$-axis as being somewhat re-scalable due to our uncertainty in the proper value of $\sigma_{\mathrm{m}}$. The important agreement with theory is the location of the minimum (indicating commensuration of $2 R_{\mathrm{c}}^{*}$ and the SAW wavelength), which is relatively independent of $m^{*}$ up to at least $m^{*} \approx 15 m_{\mathrm{b}}$.

\subsection{Coulomb Drag}

In Coulomb drag experiments ${ }^{75}$, two 2 DEGs are positioned close to each other and interact only via Coulomb forces. A current $I$ driven in once layer induces a voltage $V$ in the other layer, and thus one can define a drag resistivity $\rho_{d}=V / I$. To lowest order in the interaction between the layers the drag can 
be written as $^{76,77}$

$$
\rho_{d}=\frac{\hbar^{2}}{4 \pi T n_{1} n_{2} e^{2}} \int \frac{d \mathbf{q}}{(2 \pi)^{2}} q^{2}|U(q, \omega)|^{2} \int_{0}^{\infty} d \omega \frac{\left[\Pi_{00}^{v}\right]_{1}(\mathbf{q}, \omega)\left[\Pi_{00}^{v}\right]_{2}(\mathbf{q}, \omega)}{\sinh ^{2}(\hbar \omega /(2 T))}
$$

where $T$ is the temperature, $n_{i}$ is the density in layer $i,\left[\Pi_{00}^{v}\right]_{i}$ is the densitydensity component of the polarization in layer $i$ and $U$ is the screened interlayer interaction ${ }^{c}$. We note that the form of Eq. 213 requires that $\rho_{D}$ must vanish as $T \rightarrow 0$.

Since we have simple prescriptions (RPA or other approximations) for calculating $\Pi^{v}$, we can go ahead and use this formula to calculate predictions for Coulomb drag experiments. At filling fraction $\nu_{1}=\nu_{2}=\frac{1}{2}$, as we discovered in section 3 above, $\Pi^{v}$ is expected ${ }^{d}$ to have a pole at $\omega \sim i q^{3}$.

Plugging the form of $\Pi^{v}$ into the above expression, we find that the $q$ integral is dominated by the region near the pole $q_{0} \sim \omega^{1 / 3}$. The $\omega$ integral is then dominated by $\omega \sim T$ so we obtain the result 77,78

$$
\rho_{d} \sim T^{4 / 3}
$$

for $\nu_{1}=\nu_{2}=1 / 2$. We compare this to the result for drag between two electron gases in zero field which scales as $\rho_{d} \sim T^{2}$. It should be noted that the unusual power law form for the $\nu=\frac{1}{2}$ drag is a direct reflection of the overdamped mode. The coefficient of the scaling law (Eq. 214), as well as sub-leading corrections have also been calculated in reference 77 within the Chern-Simons theory.

Experiments by Eisenstein et al ${ }^{75}$ have measured $\rho_{d}$ for the $\nu_{1}=\nu_{2}=\frac{1}{2}$ state. For temperatures above $100 \mathrm{mK}$, the $T^{4 / 3}$ prediction seems to agree fairly well with experiment. Furthermore, the magnitude (the coefficient of $T^{4 / 3}$ ) agrees reasonably well with the prediction of Ref. 77. Agreement away from $\nu=\frac{1}{2}$ remains uncertain at the present.

At lower temperature $(T<100 \mathrm{~m} K)$, results of these experiments are extremely puzzling. Most notably, $\rho_{d}$ does not always go to zero as $T$ goes to zero, and thus cannot be described by Eq. 213. One possibility is that the interlayer interaction (although it may be weak) must be treated beyond second order - and perhaps may require nonperturbative treatment. This may also suggest the formation of a new type of state at low temperature with interlayer coherence ${ }^{79}$.

\footnotetext{
${ }^{c}$ As discussed in reference 77 one must be careful to properly screen both the inter and intra-layer interactions.

${ }^{d}$ Note that here we have a short range Coulomb interaction in each layer due to the screening of the other layer.
} 


\subsection{Activation Energy}

One particularly significant set of experiments were measurements of activation gaps $^{82,83}$ in the Jain series of fractions $\nu=\frac{p}{2 p+1}$. In these experiments, the longitudinal resistivity $\rho_{x x}$ is measured as a function of temperature in the middle of a plateau and is fit to an exponential form $\rho_{x x} \sim \exp \left(-E_{g} /(2 T)\right)$ over some range of temperatures. It should be noted that at high temperatures, the transport is not expected to be activated, and at sufficiently low temperatures, a variable-range hopping form is expected to be more appropriate ${ }^{81}$. There is thus always some uncertainty in the value of the fitted gap. It is found that near $\nu=\frac{1}{2}$, the gaps are given roughly by

$$
E_{g}=\alpha \Delta B-\Gamma
$$

with some dimensioned constant $\alpha$ (which may have a weak $B$ dependence). The prediction of composite fermion theory, would give ${ }^{e}$

$$
E_{g}=\hbar \Delta \omega_{\mathrm{c}}^{*}=\hbar e \Delta B /\left(m^{*} c\right)
$$

Thus, the experimental measurement of $\alpha$ is thought to be a measurement of the effective mass. We note that although an offset $\Gamma$ is also found for case of electron cyclotron gaps near zero magnetic field ${ }^{82,83}$, the current understanding of the effects of disorder in the Chern-Simons system is quite crude, and there is no theoretical derivation of the form of Eq. 215.

\subsection{Shubnikov-deHaas Oscillations}

Other sets of experiments ${ }^{84,83}$ have measured the temperature dependences of the resistance oscillations around $\nu=\frac{1}{2}$. Analogous to the situation around zero field, one expects to see oscillations in the conductivity corresponding to the filling of successive Landau levels where the disorder is strong enough to prevent a quantum Hall state from fully forming. There is, however, a great deal of difficulty in analyzing this data.

For the case of electrons near zero magnetic field, one can typically fits the experimental result to the Ando (Landau-Koshelev) formula ${ }^{85}$

$$
\Delta \rho_{x x}=\frac{X}{\sinh X} \exp \left(\frac{-\pi}{\omega_{\mathrm{c}}^{*} \tau}\right) \cos \left[2 \pi\left(\nu-\frac{1}{2}\right)\right]
$$

${ }^{e}$ As discussed in section $6.3, m^{*}$ formally diverges as $\log \Delta B$ as one approaches $\nu=\frac{1}{2}$. It is suspected, however, that this weak divergence would only be important very close to $\nu=\frac{1}{2}$ and is probably not observable in any experimental system. We also note that following the form of Eq. 171, we expect that $m^{*}$ will might scale as $1 / \sqrt{B}$ which would become more obvious farther away from $\nu=\frac{1}{2}$. 
where $X=2 \pi^{2} k_{b} T /\left(\hbar \omega_{\mathrm{c}}^{*}\right)$ and $\omega_{\mathrm{c}}^{*}=e B /\left(m^{*} c\right)$. This form has been derived for the case of electrons near zero magnetic field. By fitting experimental data to this form, one can extract both an effective mass $m^{*}$ and a scattering time $\tau$.

Many groups have assumed that this Ando form should also hold for the oscillations near $\nu=\frac{1}{2}$, where $B$ is replaced by $\Delta B$ and $\nu$ replaced by $\nu_{\mathrm{CF}}=p=\nu /(2 \nu-1)$. It is found that this two parameter fit can match the data over a reasonable range of temperatures if $m^{*}$ is allowed to vary as a function of filling fraction. However, it is not at all clear that this form is appropriate. Semiclassical calculations ${ }^{86}$ suggests that the argument of the exponential $\pi /\left(\tau \Delta \omega_{\mathrm{c}}^{*}\right)$ should actually be replaced by $\left[\pi /\left(\tau \Delta \omega_{\mathrm{c}}^{*}\right)\right]^{4}$. This unusual form is a result of the dephasing caused by the Chern-Simons gauge field. The experimental data can indeed be fit to this form also. However within this semiclassical picture, when one tries to estimate the scattering time (based on the supposed strength of the disorder), one obtains a result that differs from experiment by a very large factor.

Perhaps the most serious problem with this interpretation of the data is that the effect of long scale disorder is extremely important in high ${ }^{f}$ magnetic fields ${ }^{71,87}$ essentially mixing the effects of local $\rho_{x x}$ and $\rho_{x y}$. Thus, a macroscopic measurement of the resistivity may not probe the local longitudinal resistivity at all.

\subsection{Geometric Experiments}

As discussed at length above, one of the most surprising features of the composite fermion theory is the emergence of the very large length scale corresponding to the composite fermion cyclotron radius $R_{\mathrm{c}}^{*}=\hbar k_{\mathrm{F}} c /(e \Delta B)$ near filling fraction $\nu=\frac{1}{2}$. Several experiments (in addition to the SAW experiments) have attempted to observe this length scale directly by looking for the commensuration of the cyclotron orbit with an externally defined static structure. These experiments should be considered to be measurements of $k_{\mathrm{F}}$. Several of these experiments include anti-dot experiments ${ }^{88}$ and ballistic electron focusing ${ }^{90,89,91}$. These experiments do indeed observe the existence of the scale $R_{\mathrm{c}}^{*}$ in agreement with theory. In all of these geometric resonance experiments it should be noted, that although the theory of ballistic fermion motion has been reasonably well developed for electrons near zero field ${ }^{92}$, the theoretical analysis ${ }^{93,89}$ near $\nu=\frac{1}{2}$ has been performed at the mean field level only in a semiclassical approximation. However, the agreement with these crude

\footnotetext{
${ }^{f}$ In sufficiently low magnetic fields $\rho_{x y}$ is typically small enough not to influence $\rho_{x x}$ greatly, and these long scale inhomogeneities are much less important.
} 
calculations seem reasonable.

\section{Last Words}

Throughout this review we have maintained the theme of describing the even denominator quantum Hall states as unusual Fermi liquids. Starting with the mean field solution, we treated the Chern-Simons interaction at RPA level in section 3 and found that although many of the results we found were correct (DC Hall conductivity, existence of overdamped mode), the energy scales were incorrect. Attempts to systematically correct this using perturbation theory in section 6 were plagued with divergences for $\nu=\frac{1}{2 m}$. However, a detailed study of these divergences (and where they cancel) encourages us to believe that a Landau Fermi liquid approach may be a reasonably justified approximation. We construct such a Landau approach in section 4 and 5 and arrive at a picture of a quasiparticle with highly renormalized (but finite) effective mass, and magnetization equal to one Bohr magneton in the limit of large cyclotron energy. In section 7 we turn to the wavefunction picture of $\nu=\frac{1}{2 m}$ and develop the picture of the neutral dipole composite fermion. In the end, we discover that this new picture is quite equivalent to the Chern-Simons approach. Finally, in section 8 we have looked briefly at some of the experiments.

Although this review has covered a great deal of ground, it has left even more ground uncovered. Certainly there are large topics of composite fermion physics that we have not even touched on. Some of the more glaring omissions (such as the physics of composite fermion edge states ${ }^{94}$ and the very large number of experiments that have not already been mentioned here ${ }^{24}$ ) will be addressed by other chapters of this book. However, even counting these works, we have still not discussed many of the more interesting topics in compositefermion physics.

One particularly interesting theoretical topic that has been neglected is the calculation of one electron Green's function and the physics of tunneling into composite fermion systems ${ }^{95}$. Also, we have not said a single word about the spin of the composite fermion ${ }^{96}$. Nor have we said much about finite temperatures. Yet another direction that seems particularly exciting at the moment is the question of whether the composite fermion Fermi sea is unstable to forming a paired ${ }^{a}$ (BCS-like) state ${ }^{80,63}$.

There are certainly many other works that that we could examine at this point, and we would never come to the end of this chapter. Even more, we could fill several books mulling over the many outstanding questions in the

\footnotetext{
${ }^{a}$ One such possible state is the so-called Pfaffian ${ }^{80}$ which is unusual in that it has exotic nonabelian statistics.
} 
field. Needless to say, composite fermions will keep many of us busy for years to come.

\section{Acknowledgments}

Most of what I know about this subject is a result of my collaborations with Bertrand Halperin and Ady Stern. It is a pleasure to acknowledge this fruitful collaboration. In preparing this review, I have also benefited from discussions with many people including (but not limited to) R. Shankar, G. Murthy, Y. B. Kim, R. L. Willett, S. Kivelson, J. K. Jain, R. K. Kamilla, and N. d'Ambrumenil.

This work was supported by Lucent Technology, NSF Grants No. DMR94-16910 and DMR-95-23361, by the ISI foundation, and by the ESPRIT 8050 Small Structures program.

\section{A Noninteracting Response Functions in Zero Field}

For noninteracting fermions of mass $m^{*}$ in zero magnetic field, the currentcurrent correlator (Eq. 141 or Eq. 140) can be calculated to yield expressions given in Eqs. 2.18-2.19 of Ref. 14. These integrals can be evaluated exactly to yield the result

$$
\begin{aligned}
& K_{00}(q, \omega)=\frac{m^{*}}{2 \pi Q^{2}}\left(A_{-} B_{-}-A_{+} B_{+}\right) \\
& K_{11}(q, \omega)=\frac{1}{6 \pi Q^{2} m^{*}}\left(-Q^{2}+A_{+} B_{+} C_{+}-A_{-} B_{-} C_{-}\right)
\end{aligned}
$$

where $Q=q / k_{\mathrm{F}}, \Omega=\left(\omega+i 0^{+}\right) /\left(q v_{\mathrm{F}}^{*}\right), \quad A_{ \pm}=\Omega \pm Q^{2} / 2, \quad B_{ \pm}=1-$ $\sqrt{1-Q^{2} / A_{ \pm}^{2}}$, and $C_{ \pm}=1-A_{ \pm}^{2} / Q^{2}$.

It will be useful to write down the long wavelength low frequency limit of these results. For $q \ll k_{\mathrm{F}}$ and $\omega \ll q v_{\mathrm{F}}^{*}$ the expressions reduce to

$$
\begin{aligned}
& K_{00}^{0}=\frac{m^{*}}{2 \pi}\left(1+\frac{i \omega}{q v_{\mathrm{F}}^{*}}+\ldots\right) \\
& K_{11}^{0}=\frac{-q^{2}}{24 \pi m^{*}}+\frac{i \omega}{q} \frac{2 n_{\mathrm{e}}}{k_{\mathrm{F}}}+\ldots
\end{aligned}
$$

(And clearly, in zero field, the off-diagonal components should be zero).

It is sometimes convenient to use an approximate Boltzmann approach to calculate the noninteracting response function. Such an approach is discussed 
in detail in section 4 in the context of Fermi liquid theory. Since such a calculation is particularly simple, we will demonstrate it here.

The transport equation in zero magnetic field with all Fermi liquid coefficients set to zero (so $\delta \epsilon_{1}=0$ ) and the scattering term taken to zero (or $\tau$ taken to $\infty)$ is given by $\left(-i \omega+i q v_{\mathrm{F}}^{*} \cos \theta\right) \nu(\theta)=-e \mathbf{E} \cdot \hat{\mathbf{n}}(\theta)$ which is just Eq. 93 rewritten. The function $\nu$ is then given by

$$
\nu(\theta)=\frac{\frac{-e}{i q v_{\mathrm{F}}^{*}}\left(E_{x} \cos \theta+E_{y} \sin \theta\right)}{-\Omega+\cos \theta}
$$

with $\Omega=\frac{\omega}{q v_{\mathrm{F}}^{*}}$. Integrating this expression in Eq. 82 to obtain $\nu_{ \pm 1}$ and hence the current via Eqs. 86 and 87, yields the conductivity at finite frequency and wavevector

$$
\begin{aligned}
& \sigma_{x x}^{0}=\frac{i k_{\mathrm{F}} e^{2}}{q 2 \pi \hbar} \Omega\left[\frac{1}{\sqrt{1-\frac{1}{\Omega^{2}}}}-1\right] \\
& \sigma_{y y}^{0}=\frac{i k_{\mathrm{F}} e^{2}}{q 2 \pi \hbar} \Omega\left[1-\sqrt{1-\frac{1}{\Omega^{2}}}\right] .
\end{aligned}
$$

and of course the off diagonal conductivity is zero in zero magnetic field. Note that $\sigma$ can be written as $q^{-1}$ times a function only of $\Omega$. Expressions for Boltzmann conductivity in finite magnetic field ${ }^{38}$ are given explicitly in Refs. 16 and 14 for example.

To approximately include disorder to the Boltzmann approach one simply analytically continues $\omega \rightarrow \omega+i / \tau$. However as discussed in section 4.1.4 this is a non current conserving scattering model. For most purposes, however, the difference between results of different scattering models is small.

Using $K^{0}=T \sigma^{0} T$ (analogous to Eq. 48 and 56 where $v=0$ ) we can obtain an approximate expression for the response of electrons in zero field. We note that at finite wavevector but very low frequency, the Boltzmann approach and the exact expression do not agree. This is a reflection of the fact that the Boltzmann equation does not model the static compressibility of the system correctly, nor does it properly obtain the diamagnetic term ${ }^{b}$. At slightly higher frequencies however, the Boltzmann expression is quite accurate.

\footnotetext{
${ }^{b}$ This inaccuracy of the Boltzmann equation was the motivation for redefining the conductivity in Ref. 14 such that the Boltzmann equation correctly obtains the low frequency limit.
} 


\section{B RPA in yet another language}

An RPA treatment of the Chern-Simons interaction was used in earlier works on anyon superconductivity ${ }^{10,11}$. These works derive the RPA equations explicitly from a perturbative approach and obtain a prescription that, although seemingly different, is precisely equivalent to the HLR form of the RPA. The different appearance stems from an apparently different treatment of diamagnetic terms. In this appendix we will show how the HLR RPA can be derived from the earlier form of RPA given in Refs. 10 and 11 by a simple regrouping of the perturbation series.

In Refs. 10 and 11, the diamagnetic term is separated out of the noninteracting response function as (See Eq. 143) $K_{0}=D_{0}+E$. In this approach, an RPA prescription for the full current-current correlator is given as

$$
\begin{aligned}
D^{-1} & =D_{0}^{-1}-W \\
W^{-1} & =(C+V)^{-1}-E
\end{aligned}
$$

where $V$ is the Coulomb interaction and $C$ is the Chern-Simons interaction ${ }^{c}$. From this full correlation function, the full response is given by

$$
\begin{aligned}
K & =\Lambda+E \\
\Lambda & =(1+E W) D(1+W E)
\end{aligned}
$$

Noting that Eq. B.1 is equivalent to $D=D_{0} W D+D_{0}$ can rewrite Eqs. B.3 and B.4 as

$$
\begin{aligned}
K & =\left(D_{0} W D+D_{0}+E W D\right)(1+W E)+E \\
& =\left(D_{0}+E\right)(W D W+W)\left(W^{-1}+E\right)
\end{aligned}
$$

where we have used the fact that $E W E=0$. Using $K_{0}=D_{0}+E$ as well as the fact that Eq. B.1 implies that $\left(W^{-1}-D_{0}\right)^{-1}=W D W+W$ we obtain

$$
\begin{aligned}
K & =K_{0}\left(W^{-1}-D_{0}\right)^{-1}\left(W^{-1}+E\right) \\
& =K_{0}\left([C+V]^{-1}-E-D_{0}\right)(C+V)^{-1} \\
& =K_{0}\left([C+V]^{-1}-K_{0}\right)[C+V]^{1} \\
& =K_{0}\left(1-[C+V] K_{0}\right)^{-1} \\
& =\left(\left[K^{0}\right]^{-1}-C-V\right)^{-1}
\end{aligned}
$$

which is precisely the HLR prescription given by Eq. 66 (except for - signs which are a result of having used a different convention for $e$ ).

${ }^{c}$ In Refs. 10, 11 the Coulomb interactions is set to zero. Also in these references $W$ is called $V$. 
1. For a basic review of both the integer and fractional quantized Hall effects, see R. E. Prange and S. M. Girvin eds. The Quantum Hall Effect, second edition (Springer-Verlag, New York, 1990).

2. An excellent reprint collection of important papers on the theory of quantum Hall physics (up to 1991) is given in M. Stone ed. Quantum Hall Effect, (World Scientific, 1992).

3. For reviews on recent progress in integer and fractional quantum Hall effect, see S. Das Sarma and A. Pinczuk eds. New Perspectives in Quantum Hall Effect (Wiley, NY, 1996).

4. Another review of fractional quantum Hall physics is given by $\mathrm{T}$. Chakraborty and P. Pietiläinen, The Fractional Quantum Hall Effect, (Springer-Verlag, NY, 1995); 2 ed.

5. J. K. Jain, Phys. Rev. Lett. 63, 199 (1989).

6. J. K. Jain, Adv. Phys. 41, 105 (1992), and therein. See also the chapter by Jain in Ref. 3 and the Chapter by Jain and Kamilla in this book.

7. S. M. Girvin and A. H. MacDonald, Phys. Rev. B58, 1252 (1987); S. M. Girvin in Ref. 1.

8. N. Read, Phys. Rev. Lett. 62, 86 (1988).

9. S. C. Zhang, T. H. Hansson, and S. Kivelson, Phys. Rev. Lett. 62, 82 (1988); S.-C. Zhang, Int. J. Mod. Phys. 6, 25 (1992) and references therein;

10. Y. H. Chen, F. Wilczek, E. Witten, and B. I. Halperin, Int. J. Mod. Phys. 3, 1001 (1989); See also appendix B for a discussion of how the RPA in these works are related to the RPA used in HLR.

11. A. L. Fetter, C. B. Hanna, and R. B. Laughlin, Phys. Rev. B39, 9679 (1989); Q. Dai et al, Phys. Rev. B46, 5642 (1992); See also appendix B for a discussion of how the RPA in these works are related to the RPA used in HLR.

12. For a more thorough historical review of Anyons, see F. Wilczek, Fractional Statistics and Anyon Superconductivity (Singapore, World Scientific, 1990).

13. A. Lopez and E. Fradkin, Phys. Rev. B 445246 (1991); ibid. 47, 7080 (1993); It should be noted that Lopez and Fradkin call the RPA a "semiclassical" approximation. See also the chapter in this book by Lopez and Fradkin.

14. B. I. Halperin, P. A. Lee, and N. Read, Phys. Rev. B 47, 7312 (1993); This paper is known as "HLR" to most people. A recent review of progress on $\nu=\frac{1}{2}$ is given by B. I. Halperin in Ref. 3.

15. V. Kalmeyer and S.-C. Zhang, Phys. Rev. B 46, 9889 (1992).

16. S. H. Simon and B. I. Halperin, Phys. Rev. B 48, 17386 (1993). 
17. S. H. Simon, A. Stern, and B. I. Halperin, Phys. Rev. B 54, R11114 (1996); See also reference 18.

18. S. H. Simon, J. Phys. Cond. Matt., 48, 10127 (1996).

19. R. B. Laughlin, Phys. Rev. B23, 5632 (1981); B. I. Halperin, Phys. Rev. B25, 2185 (1982).

20. K. von Klitzing, G. Dorda, and M. Pepper, Phys. Rev. Lett. 45, 494 (1980).

21. See for example, E. R. Cohen and B. N. Taylor, Physics Today, 43, august part II, (1990); See also M. Cage in Ref. 1.

22. D. C. Tsui, H. L. Stormer, and A. C. Gossard, Phys. Rev. Lett. 48, 1559 (1982).

23. R. B. Laughlin, Phys. Rev. Lett. 50, 1935 (1983); see also the Chapter by R. B. Laughlin in Ref. 1.

24. A thorough review of experimental work on composite fermion physics has been given by R. L. Willett, Adv. Phys. 46, 447 (1997); See also the Chapters by R. L. Willett and J. H. Smet in this book.

25. B. I. Halperin, Phys. Rev. Lett. 521583 (1984); erratum 522390 (1984);

26. F. D. M. Haldane, Phys. Rev. Lett. 51, 605 (1983); See also the Chapter by F.D.M. Haldane in Ref. 1.

27. W. Kohn, Phys. Rev. 123, 1242 (1961).

28. See for example G. Fano, F. Ortolani, and E. Columbo, Phys. Rev. B34, 2670 (1986) for examples of exact diagonalizations. See also Refs. 6, 42, $61,41,23,63$ and 57

29. D. Pines and P. Nozières, Theory of Quantum Liquids, v. I, (Benjamin, New York, 1966);

30. P. Nozières, Theory of Interacting Fermi Systems (Benjamin, New York, 1964).

31. E. M. Lifshitz and L. P. Pitaevskii, Statistical Physics, Part 2(Pergamon, NY, 1980); G. Mahan, Many Particle Physics, (Plenum, NY, 1981).

32. A. Stern, Phys. Rev. B50, 10092 (1995).

33. R. Shankar, Rev. Mod. Phys. 66, 129 (1994).

34. W. M. Brinkman, P. M. Platzman, and T. M. Rice, Phys Rev 174, 495 (1968).

35. T. K. Lee and J. J. Quinn, Phys. Rev. Lett. 11, 2144 (1975).

36. P. M. Platzman, W. M. Walsh, Jr., and E. N. Foo, Phys. Rev. 173, 689 (1968); P. M. Platzman and W. M. Walsh, Jr., Phys. Rev. Lett. 19, 514 (1967).

37. A. D. Mirlin and P. Wölfle, Phys. Rev. Lett 78, 3717 (1997).

38. M. H. Cohen, M. J. Harrison, and W. J. Harrison, Phys. Rev. 117, 937 
(1960).

39. S. H. Simon and A. Stern, unpublished.

40. V. P. Silin, Sov. Phys. JETP 6, 387 (1957); ibid 6, 985 (1958); ibid 6, 995; 7486 (1958). See Electron Liquid Theory of Normal Metals, Proceedings of the Lebedev Physics Institute v. 174 Supplement 2, ed. V. P. Silin, (Nova Science, Commack N.Y., 1988) for more modern references.

41. S. H. Simon and B. I. Halperin. Phys. Rev. B50, 1807 (1994); S. He, S. H. Simon, and B. I. Halperin, Phys. Rev. B50, 1823 (1994).

42. E. Rezayi, R. Morf, N. Read, N. d'Ambrumenil, and S. H. Simon, unpublished.

43. A. Stern and B. I. Halperin, Phys. Rev. B 52, 5890 (1995); Surf. Sci. 36142 (1996).

44. Y. B. Kim, A. Furusaki, X.-G. Wen, and P. A. Lee., Phys. Rev. B 50, 17917 (1994)

45. B.L. Altshuler, L. Ioffe and A.J. Millis, Phys. Rev. B 50, 14048 (1994).

46. D.V. Kveshchenko and P.C.E. Stamp, Phys. Rev. Lett. 71, 2118 (1993); Phys. Rev. B49, 5227 (1994).

47. C. Nayak and F. Wilczek, Nuc. Phys. B 417, 359 (1994); J. Gan and E. Wong, Phys. Rev. Lett. 71, 4226 (1993); J. Polchinski, Nucl. Phys. B 422, 617 (1994).

48. H.J. Kwon, A. Houghton and J.B. Marston, Phys. Rev. Lett. 73, 284 (1994); Phys. Rev. B52, 8002 (1995); P. Kopietz, Bosonization of Interacting Fermions in Arbitrary Dimensions, (Springer, Berlin, 1997).

49. R. Shankar and G. Murthy, Phys. Rev. Lett. 79, 4437 (1997). See also the chapter by R. Shankar in the present book. (preprint, condmat/9802244).

50. V. Pasquier and F. D. M. Haldane, preprint cond-mat/9712169.

51. D.-H. Lee, preprint cond-mat/9709329.

52. B. I. Halperin and A. Stern, cond-mat/9802051.

53. A. Stern, F. Von Oppen, S. H. Simon, and B. I. Halperin, preprint.

54. Y. B. Kim, P. A. Lee, X.-G. Wen and P. C. E. Stamp, ibid. 51, 10779 (1995).

55. Y. B. Kim, P. A. Lee, and X.-G. Wen, Phys. Rev. B 5217275 (1995).

56. Y. B. Kim and P. A. Lee, Phys. Rev. B 54, 2715 (1996).

57. R. Morf and N. d'Ambrumenil, Phys. Rev. Lett. 74, 5116 (1995).

58. N. Read, Semi. Cond. Sci. Tech. 9, 1859 (1994); Surf. Sci., 361/362, 7, (1996).

59. B. I. Halperin, Helv. Phys. Acta 56, 75 (1983).

60. S. A. Trugman and S. Kivelson, Phys. Rev. B31, 5280 (1985); V. L. Pokrovsky and A. L. Talapov, J. Phys. C18 L691 (1985). 
61. E. H. Rezayi and N. Read, Phys. Rev. Lett. 72, 900 (1994).

62. S. M. Girvin and T. Jach, Phys. Rev. B 29, 5617 (1984); see also S. Kivelson, C. Kallen, D. P. Arovas, and J. R. Schrieffer, Phys. Rev. B36, 1620 (1987).

63. F. D. M. Haldane, unpublished; F. D. M. Haldane and E. Rezayi, unpublished.

64. R. K. Kamilla and J. K. Jain, preprint cond-mat/9704031.

65. See S.L. Sondhi and S. A. Kivelson, Phys. Rev. B 46, 13319 (1992).

66. A. Lopez and E. Fradkin, Phys. Rev. Lett, 69, 2126 (1992).

67. R. L. Willett, et. al, Phys. Rev. Lett. 65, 112 (1990)

68. R. L. Willett, et. al, Phys. Rev. B47, 7344 (1993).

69. S. H. Simon, Phys. Rev. B, 54, 13878 (1996).

70. See for example, K. A. Ingebrigtsen, J. Appl. Phys. 40, 2681 (1969).

71. S. H. Simon and B. I. Halperin, Phys. Rev. Lett. 73, 32781994 ; S. H. Simon and N. R. Cooper, Phys. Rev. B 56, R7116, 1997; N. R. Cooper, B. I. Halperin, C.-K. Hu and I. Ruzin. Phys. Rev. B55, 4551 (1997); I. M. Ruzin, N. R. Cooper, and I. Ruzin, Phys. Rev. B53, 1558 (1996).

72. R. L. Willett, et. al, Phys. Rev. Lett. 71, 3846 (1993).

73. R. L. Willett, K. W. West, and L. N. Pfeiffer, Phys. Rev. Lett. 75, 2988 (1995).

74. S. H. Simon, unpublished; S. H. Simon, Minnesota conference on compressible Hall states, 1996.

75. M. P. Lilly et al, preprint cond-mat/9710041.

76. L. Zheng and A. H. MacDonald, Phys. Rev, B48, 8203 (1993); A. Kamenev and Y. Oreg, Phys. Rev. B52, 7516 (1995).

77. I. Ussishkin and A. Stern, Phys. Rev. B, 56, 4013 (1997); Physica E 1, 176 (1997).

78. Y. B. Kim and A. J. Millis, preprint cond-mat/9611125; S. Sakhi, Phys. Rev. B 56, 4098 (1997).

79. I. Ussishkin and A. Stern, unpublished; N. Bonesteel, I. A. McDonald, and C. Nayak, Phys. Rev. Lett. 77, 3009 (1996).

80. G. Moore and N. Read, Nucl. Phys. B 360 (1991), 362; M. Greiter, X.-G. Wen, and F. Wilczek, Nucl. Phys. B 374 (1992), 567.

81. See for example, D. G. Polyakov and B. I. Shklovskii, Phys. Rev. Lett. 73, 1150 (1994).

82. R. R. Du et al, Phys. Rev. Lett. 70, 2944 (1993).

83. R. R. Du et. al, Phys. Rev. Lett. 73, 3274 (1994); H. C. Manoharan et. al; Phys. Rev. Lett. 73, 3270 (1994); See also Ref. 73.

84. D. R. Leadly et. al, Phys. Rev. Lett. 72, 1906 (1994); Surf. Sci. 361, 22 (1996). 
85. T. Ando, J. Phys. Soc. Japan 37, 1233 (1974) .

86. A. G. Aronov, E. Altshuler, A. D. Mirlin and P. Wölfle, Phys. Rev. B 52, 4708 (1995); A. D. Mirlin, E. Altshuler, and P. Wölfle, Ann Phys. (Leipzig), 5, 281 (1996); See also P. A. Lee, E. Mucciolo, and H. Smith, Phys. Rev. B54 8782 (1996).

87. A. D. Mirlin, D. G. Polyakov, and P. Wölfle, Phys. Rev. Lett. 80, 2429 (1998).

88. W. Kang, et al, Phys. Rev. Lett. 71, 3850 (1993).

89. J. H. Smet et al, Phys. Rev. Lett. 77, 2272 (1996); See also the chapter in this book by J. Smet.

90. V. J. Goldman, B. Su, and J. K. Jain, Phys Rev Lett 72, 2065; See 24 for a discussion of a dissenting opinion.

91. C. T. Lang, et. al, Phys Rev. B53, 7596 (1996).

92. See for example, C. W. J. Beenakker and H. Van Houten, in Solid State Physics v. 44, eds. H. Ehrenreich and D. Turnbull (1991); and references therein.

93. See for example, R. Fleischmann, et al, Europhys. Lett., 36, 167 (1996).

94. L. Brey, Phys. Rev. B50, 11861 (1994); D. B. Chklovskii, Phys. Rev. B51, 9895 (1995); See also the Chapter by G. Kirczenow in this book.

95. Y. B. Kim and X. G. Wen, Phys. Rev. B50, 8078 (1994); S. He, P. M. Platzman, and B. I. Halperin, Phys. Rev. Lett. 71, 777 (1993).

96. R. R. Du et al, Phys. Rev. Lett. 75, 3926 (1995). 